\title{
Christian Sappok
}

\section{Die Bedeutung des Raumes für die Struktur des Erzählwerks}

\author{
Aufgezeigt an Beispielen \\ aus der polnischen Erzählliteratur
}

Verlag Otto Sagner München · Berlin · Washington D.C.

Digitalisiert im Rahmen der Kooperation mit dem DFG-Projekt „Digi20“

der Bayerischen Staatsbibliothek, München. OCR-Bearbeitung und Erstellung des eBooks durch den Verlag Otto Sagner:

http://verlag.kubon-sagner.de

( $)$ bei Verlag Otto Sagner. Eine Verwertung oder Weitergabe der Texte und Abbildungen, insbesondere durch Vervielfältigung, ist ohne vorherige schriftliche Genehmigung des Verlages unzulässig. 


\section{SLAVISTISCHE BEITRÄGE}

Unter Mitwirkung von M. Braun, Göttingen + P. Diels, München $\cdot J$. Holthusen, München - E. Koschmieder, München - W. Lettenbauer, Freiburg/Br. - J. Matl, Graz

F. W. Neumann, Mainz - K.-H. Pollok, Regensburg - L. Sadnik-Aitzetmüller, Graz $\cdot J$. Schütz, Erlangen

HERAUSGEGEBEN VON + A. SCHMAUS, MÜNCHEN Technische Redaktion: P. Rehder, München

Band 47 
CHRISTIAN SAPPOK

\section{DIE BEDEUTUNG DES RAUMES FÜR DIE STRUKTUR DES ERZÄHLWERKS}

aufgezeigt an Beispielen aus der polnischen Erzählliteratur

VERLAG OTTO SAGNER - MÜNCHEN

1970 
Copyright by Verlag Otto Sagner, Munchen 1970 Abtellung der Fa. Kubon u. Sagner, München Druck: Kubon u. Sagner

8 München 13, Heßstr. 39 
Seite

Einleitung

A. Theoretischer Teil

1. Das Raumproblem in der Sprache, beim Erzählen und im literarischen Kunstwerk

2. Die Zuordnung von sprachlichem Zeichen und räumlicher Gegebenheit

3. Sukzession und Zeit im Erzählwerk

4. Das Feld als Grundlage der Raumdarstellung

5. Die Rolle des Raumes im EntstehungsprozeB der literarischen Fiktion

6. Die werkimmanente Raumgenese als spezifisch literarische Erscheinungsform des Raumes

B. Anwendungsbeispiele

1. Inkongruenz von Wortmaterial und Raumerlebnis als Mittel der Spannungserzeugung in Sienkiewicz' "Quo vadis"

2. Merkmale des Raumes und Charakteristik der Figur in Sienkiewicz' "Szkice węglem" und Prus' "Lalka"

3. Die umgekehrte Perspektive in Paris-Kapitel der "Lalka"

4. Integration von Schauplatz und Handlung in Reymonts "Chłopi"

5. Der symbolische Raum in Żeromskis "Przedwiośnie"

6. Die Erzählphase als homogenes Glied in der kontinuierlichen Genese der werkimmanenten Welt in Dq̨browskas "Noce 1 dnie"

7. Die Entmedialisierung der Sprache in Iwaszkiewicz' "Miyn nad Utratą"

8. Der Ubergang des Zeichenkontextes in eine selbständige räumliche Situation in Bruno Schulz' "Sklepy cynamonowe" und "Emeryt" 
9. Das Zeigwort als Orientierungssignal für die Figur und die zeitliche Desorientierung des Lesers in Andrzejewskis "Bramy raju"

10. Das Prinzip der revertierten Metaphorik zur Umstrukturierung der Sphären des Dinglichen und des Begrifflichen in Andrzejewskis "Bramy raju"

Zusammenfassung

Iiteraturverzeichnis 
Die Bedeutung des Raumes für die Struktur des Romanes

\section{Einleitung}

Die vorliegende Arbeit zerfällt in zwei Teile, in eine theoretische Behandlung des literarischen Raumproblems und in eine Anzahl von der polnischen Erzähl-Literatur entnommenen Anwendungsbeispielen.

Dieser Zweiteilung entspricht zunächst eine Gegenüberstellung von Theorie und Praxis im üblichen Sinne, also eine Aufstellung allgemeiner Prinzipien an empirisch vorgefundenem vaterial. Dem Stand des literaturwissenschaftlichen Raumproblems und den spezifischen Erscheinungsformen des Raumes im Sprachkunstwerk entsprechend ergeben sich für die beiden Teile jedoch verschiedenartige und methodisch zu sondernde Schritte.

In der Theorie ist nämlich von der sprachwissenschaftlichen Fragestellung auszugehen, also von der Untersuchung der rein sprachlichen Mittel und ihrem Verhältnis zum räumlichen Objekt. Von der Grundlage dieser Gegebenheiten, den sprachlichen Zeichen und ihrer syntaktischen Kombination, kann man erst vordringen zu gröBeren Einheiten, zu Erzählabschnitten und Erzählwerken, wobei immer noch die Frage im Vordergrund steht, wie sich diese einem in der außersprachlichen Realität als Objekt vorliegendem Raum gegenüber verhalten. Der dritte Schritt faßt das ganze Werk ins Auge, in welchem einzelne Raumdarstellungen fakultativ vorhanden sein können. Die Prage ist dabei, wie im Rahmen der Gesamtstruktur ein Gesamtraum entsteht.

Im zweiten Teil, bei der literarischen Analyse also, liegt das Erzählen bzw. das Erzählwerk als ein abgeschlossenes vollständiges Ergebnis vor, und die oben genannten Schritte müssen in der Reihenfolge, die durch das Werk bestimmt wird und die für die Analyse methodisch die fruchtbarste ist, durchgeführt werden. Ein neues Moment tritt jedoch hinzu: der Raum erscheint in einem solchen Ergebnis nicht abgegrenzt und isoliert, sondern im Verband mit einer Reihe anderer Gegebenheiten, so daß man nach dem Verhältnis dieser Faktoren und ihrer für die Art des Ergebnisses spezifischen Integrationsform und der Integration dieser Faktoren 
forschen muB. Das Programm für die beiden angedeuteten Schritte sieht so aus: im ersten Teil wird die Frage nach der Anwendbarkeit des sprachlichen Mediums auf räumliche Gegebenheiten gestellt. Es zeigt sich, daß die Sprache als Medium trotz des Vorherrschens zeitlicher Relationen in ihrem System nicht versagt, sondern für ihre Darstellung verschiedene Mittel bereitstellt. Die Untersuchung dieser zeichenmäBigen Mittel in einer bestimmten Sprache ist Aufgabe der Sprachwissenschaft. Die vom Autor ausgewählten Zeichen treten dabei in Verbindung mit anderen Zeichen als gegliederte Folgen auf, wobei die Relevanz der Reihenfolge von der grammatikalischen Struktur bestimmt wird, ohne auf das Darstellungsergebnis einen Einfluß auszuüben.

Nun ist es aber nicht nur die Anzahl der adäquaten Zeichen, die für einen vorliegenden Raum ausgewählt werden muß und die ihn am anderen Ort wieder vertreten, sondern auch ihre sukzessive Anordnung, nun nicht mehr im grammatikalischen, sondern im satzübergreifenden Zusammenhang, die für das Darstellungsergebnis von Bedeutung werden kann.

Der Erzählende bildet bei der Darstellung des Raumes Sätze, in denen gleiche Stellen mit raumbezogenen Zeichen besetzt sind; und die Eigenart ihrer Abfolge bestimmt den Charakter des Raumes, indem sie sich zu einer einheitlichen Wirkung oder einem geschlossenen Ergebnis zusammenschließen. Diesen weiteren Schritt nenne ich in Abhebung von der Raumdarstellung die Raumfiktion Im Gegensatz zur ersteren handelt es sich dabei nicht um eine bloße Relevanz der Reihenfolge, sondern um die Entstehung von Einheiten zeitlich ausgedehnter Art, wie sie mit der Schaffung gröBerer sprachlicher Zusammenhänge verbunden ist. Der als Ergebnis eines solchen Sprechaktes entstandene Raum ist in seinen Merkmalen von den spezifisch sprachlichen Eigenarten des ausgewählten Wortmaterials abhängig. Dabei muß das Verhältnis der Zeichensukzession $z u$ den im Entstehen begriffenen zeitlichen Einheiten untersucht werden. Ebenso die Rolle, die dabei außersprachliche Zeitrelationen, wie die Phasen des Bewußtseinsaktes oder ähnliches spielen.

Hier ist die Grenze im Anwendungsbereich der Begriffe Sprechen und Erzählen erreicht. Die theoretische Differenzierung vollziehe 
ich unter Zuhilfenahme des Feldbegriffs. Das Ergebnis des Sprechaktes kann nur als Ganzes gesehen werden, wenn man es im Verband mit der sogenannten Sprechsituation betrachtet, also im Verband der Realitätskomponenten, die seine Bedeutung feldmäßig umgeben und ergänzen. Dieses Situationsfeld, das keine sprachliche Realisierung durch Zeichenvertretung zum Merkmal hat, kann auch beim Erzählen gefunden und nachgewiesen werden. Als Wesensmerkmal des Erzählens postuliere ich, daß die dabei wirksamen Felder nicht aus Komponenten der den Erzählenden ungenannt umgebenden Realität entstammen, sondern aus Komponenten gebildet werden, welche im Erzählzusammenhang entstanden sind und in diesem genannt bzw. nachweisbar sind.

Daß dabei auch Felder wirksam werden können, die auf spezifisch räumlichen Situationen beruhen, ohne daß der Raum expressis verbis genannt wird, ist die Grundlage der werkimmanenten Raumgenese. 


\section{A. Theoretischer Teil}

1. Das Raumproblem in der Sprache, beim Erzählen und im literarischen Kunstwerk

Der Raum wird im Erzählwerk nicht immer wieder aufs neue aus dem Nichts, an einem Ort ohne räumliche Boordinaten und Stellenwerte aufgebaut, so daß man nach diesen Grundsteinen und Fundamenten fragen müßte. Die Summe und die Kombination aller im Werk genannten, also bezeichneten räumlichen Elemente ist nicht gleich dem Raum, welcher für die Struktur dieses Werkes relevant ist. Wie diese Behauptung positiv zu formulieren ist und diese Beobachtungen methodisch in die literarische Analyse einzuordnen sind, darin liegt die Aufgabe der vorliegenden Arbeit.

Sucht man nach den Methoden, mit denen sich die Literaturwissenschaft mit dem Raumproblem auseinandersetzt, so ist es unumgänglich, das Objekt der Analyse zu differenzieren, und zwar in den rein sprachlichen Sektor, den Bereich des Erzählens, und in das Sprachkunstwerk, das in Form eines geschlossenen Erzählvorgangs vorliegt. Auch bei der Werkanalyse und der Suche nach den ästhetisch wirksamen Funktionen des Raumes ist diese Dreiteilung notwendig, da sie innerhalb eines Werkes auf einen der drei genannten Sektoren konzentriert sein können und die Beschränkung des Blickwinkels auf einen Sektor zu einer Fehlinterpretation oder zu einer Scheinproblematik führen kann. So verlüuft beispielsweise die Raumdarstellung in J. Andrzejewskis "Bramy Raju" nach den traditionellen Techniken der Personifizierung, der Metaphorik usw., während sich die originelle Raumkonstruktion erst beim Blick auf das gesamte Werk offenbart. In W.St. Reymonts Roman "Chłopi" dagegen kann man beim Blick auf den Aufbau des ganzen Werkes zwar an bestimmten Stellen eine groBangelegte Integration von Naturgeschehnissen und figuralem Handlungsablauf als eines der Strukturmerkmale feststellen, doch erst die Analyse der einzelnen literarischen Topoi, die zu Raumbildern zusammengefügt sind, zeigt, auf welche besondere Art der Autor den Raum als eine Gegebenheit sui generis für das Erzählen relevant werden läßt. Die gesonderte Formulierung des Raumproblems für die Sprache, das 
Erzählen und das Sprachkunstwerk macht erst die gewonnenen Termini zu präzisen Werkzeugen, die als heuristisches Prinzip in der Analyse Verwendung finden können.

Ich gehe zunächst auf drei Möglichkeiten der Formulierung des Raumproblems ein, die zwar jeweils Kunstwerke zum Gegenstand haben, sich im Prinzip jedoch ausschlieblich auf das sprachliche Material, also das Zeichen und seine Kombinationsmöglichkeiten mit anderen stützen. Die einflußreichste These auf diesem Sektor stammt aus Lessings "Laokoon"; er stellt in seiner Argumentation die Malerei und die Poesie einander merkmalhaft gegenüber. "Ich schlieBe so: Wenn es wahr ist, daB die Malerei zu ihrer Nachahmung ganz andere Mittel und Zeichen gebraucht als die Poesie, jene nämlich Figuren und Farben im Raum, diese aber artikulierte Töne in der Zeit, wenn unstreitig die Zeichen ein bequemes Verhältnis zu dem Gezeichneten haben müssen: so können nebeneinander geordnete Zeichen auch nur Gegenstände, die nebeneinander, oder deren Teile nebeneinander existieren, aufeinander folgende Zeichen aber nur Gegenstände ausdrücken, die aufeinander oder deren Teile aufeinander folgen". 1

Die Dominanz der Zeit, als deren Wesensmerkmal Lessing das Aufeinanderfolgen postuliert, für das sprachliche Zeichensystem steht außer Zweifel; daß die dargestellten Gegenstände, ungeachtet der Tatsache, daß sie durch sukzessiv angeordnete Zeichen vertreten werden, auch eine räumliche Umgebung haben, muß aber in gleicher Weise anerkannt werden. Ziel der folgenden Untersuchung ist der Nachweis, daß diese räumlichen Relationen der bezeichneten Gegenstände in der Art der Zuordnung von Zeichen in verschiedenem Maße berücksichtigt und auf verschiedene Art zum Ausdruck gebracht werden können. Daher kann im Erzählkunstwerk als einer kontinuierlichen und organisierten Zeichenfolge bzw. als einem in sich geschlossenen Komplex geordneter Zeichen der Raum nicht nur dargestellt werden, sondern auch durch seine ordnenden Kräfte den Vorgang der Fiktion steuern und für die Struktur des Werkes in verschiedener Hinsicht relevant werden. Das Problem des Raumes im Werk ist nicht das Verhältnis von zeit-

1 G.E. Lessing: Laokoon, in: Gesammelte Werke, 2. Band, München 1959, S. 875 . 
lichem Medium und räumlicher Gegebenheit, sondern das von einem abgeschlossenen sprachlichen Darstellungsvorgang und der Relevanz räumlicher Relationen am Darstellungsobjekt.

Wie die Vorstellung von den sukzessiv angeordneten Zeichen und der "bequemen" Zuordnung zum Darstellungsobjekt auf ein konkretes Erzählwerk anwendbar ist, will ich kurz am Beispiel Bruno Schulz skizzieren, in dessen Werk immer wieder geschlossene, thematisch verwandte Raumdarstellungen vorkommen. Schulz verwendet vorzugsweise Zeichen, die ihrerseits dem Bereich der Raumanalyse entspringen (z.B. Zone, Konstellation, Horizont usw.). Der Wissenschaftler hat nun aber ein Erzählwerk vor sich und keinen Raum. Er darf seine eigenen Begriffe nicht unbesehen auf das andersgeartete Objekt übertragen, er muß von seiner eigenen Raumkonzeption absehen, da dieser Maßstab für das Werk irrelevant ist. Schulz verwendet, wie unten gezeig t wird, raumbezogene Termini, also in erster Iinie der nachprüfbaren Analyse entstammende Begriffe und ordnet sie durch das Erzählen sukzessiv an. Doch durch eine Weiterentfaltung dieser Wörter, eine Erscheinung, die ich semantische Expansion nenne, löst sich schlieblich das sprachliche Ergebnis von der ursprünglich abzubildenden Realität, und es entsteht ein eigenständiger Raum. Die Begriffe werden zu Symbolen und damit zu konstituierenden Bausteinen eines Raumes, wie er nur auf der Grundlage dieses sprachlichen Prozesses möglich ist. Diese Besonderheit findet in der außerhalb dieses speziellen Raumes nicht denkbaren Art von Figuren und Handlungsabläufen ihren Niederschlag. Auf jeden Fall bedingt diese Doppelschichtigkeit des raumbezogenen Wortmaterials den doppelten Aspekt seiner zeitlichen Anordnung. Die Termini sind aneinandergereiht, die spezifische Reihenfolge ist für das Ergebnis irrelevant. Die gleichen Zeichen als Symbole lassen eine Sukzession entstehen, die in ihrer spezifischen Reihenfolge ausschlaggebend ist für den Charakter des Ergebnisses. Die Notwendigkeit des Hintereinanders der Zeichen wird abgelöst von einem autonomen ProzeB mit spezifischer räumlicher Wirkungsform. Lessing behandelt das Problem, welches auftaucht, wenn ein Künstler mit den Mitteln der Sprache bewußt den Raum in Angriff nimmt. Welches Problem stellt sich aber, wenn das im Raum Befindliche Hauptziel 
der Darstellung ist, jener nur als notwendige Nebenerscheinung mit einbezogen werden muB? T. Zielinski 1 geht darauf ein, indem er sich auf die Fälle stützt, in denen es gilt, parallel verlaufende Handlungsstränge zu koordinieren. Er analysiert mit Hilfe komplizierter graphischer Darstellungen Homers Technik der Beschreibung gleichzeitig stattfindender Ereignisse und schreibt: "Was für die räumlichen Zustände die Malerei, das ist für die zeitlichen Vorgänge die Poesie (im weitesten Sinne); dieser Lessingsche Grundsatz ist unverrückbar. Fragen wir nun nach den Darstellungsmitteln der Poesie dem zeitlichen Nebeneinander gegenüber, so ergibt sich, daB sie - ähnlich wie die Darstellungsmittel der Malerei gegenüber dem Körperhaften - eigentlich vollkommen versagen: Die Poesie ist die Kunst des Nacheinander schlechthin. Will sie dennoch das Nebeneinander der Darstellung bringen, so muB sie auf die Illusionsfähigkeit unseres Vorstellungsvermögens rechnen, die es ihm möglich macht, nacheinander empfangene Eindrücke als nebeneinander bestehend oder bestehen habend zu denken." 2

Hier und in einer bis in die jüngste Zeit sich anschlieBenden Analyse von Epen verschiedener Epochen fängt die Terminologie an zu verschwimen. Ein "zeitliches Nebeneinander" ist für eine Argumentation, die das Nebeneinander zur Definition des Raumes verwendet, ein Ding der Unmöglichkeit; und die Illusionsfähigkeit ist nicht eine Art Notlösung oder letzter Ausweg für das Vorstellungsvermögen, sondern das eigentliche Wesensmerkmal. Sie ist eine Tatsache, die dem künstlerischen Schaffen vorausgeht und von ihm unabhängig ist.

Dieser Gedanke von den parallelen Abläufen hat folgende Ausgangsbasis: Von einem in bestimmter Weise festgelegten Punkt sei die Lage eines Gegenstandes nach einem vorher geschaffenen Maßstab bestimmt. In diesem Maßstab erhält der Gegenstand dadurch einen Stellenwert. Dabei ist festzuhalten, daß es sich um eine künstlich geschaffene Situation handelt, wobei es Gegenstände gibt, die mit diesem Maßstab nicht wesensmäBig erfaßbar sind. Die metrische, quantifizierende Methode, die mit der Schaffung des Gegen-

1 T. Zielinski: Die Behandlung gleichzeitiger Ereignisse im antiken Epos, in: Philologus, Supplementband 8, Heft 3 (1901), S. $405-449$.

2 A.a.0. S. 414 . 
übers von einem Standpunkt im Raum und einem räumlichen Objekt verbunden ist, ist nur eine von vielen Möglichkeiten dieser Art. Ferner handelt es sich bei dieser räumlichen Perspektive um eine Orientierungsmethode, die in der Geschichte gewachsen ist und eine ganz bestimmte Entwicklung und Verbreitung mitgemacht hat. Die Einnahme des oben charakterisierten Standpunktes von seiten des Künstlers ist nicht eine im jeweiligen Einzelwerk separat und isoliert wieder ansetzbare Tatsache, sondern liegt als in der Tradition entstandene und damit bindende Voraussetzung fest. Sie wird vom Leser erwartet und übt auch dort, wo sie durchbrochen werden soll, als zunächst normale und einzig richtige Form der Raumkonzeption immer noch eine steuernde Wirkung aus.

In einem Kapitel von Prus' "Lalka", in welchem der Held Paris erlebt und das Erlebnis dieses für ihn neuen Raumes mit den ihm eigenen Mitteln beschreibt, können diese völlig unzureichenden Mittel nur dadurch ihren künstlerischen Effekt entfalten, daß eine verborgene, im Erzählverlauf nicht in Erscheinung tretende Perspektive durch die Versetzung des Lesers in den Raum mitwirkt. Diese a priori festgelegte Perspektive wird in diesem Kapitel von Prus negativ ausgewertet.

Das Problem der Parallelität zweier verschiedener Handlungen, wie es Zielinski als Ausgangspunkt wählt, muß nun durch die Frage eingeschränkt und spezifiziert werden, wann, in welcher Situation eine solche Gegebenheit der zeitlichen Parallelität überhaupt erst auftaucht. Welche Voraussetzungen sind nötig, die diese Erscheinung - auch außerhalb des sprachlich eingeengten Bereichs - bedingen? Eine genaue Untersuchung der Bedingungen, wann an verschiedenen Orten stattfindende Vorgänge als zeitlich parallel anzusehen sind, soll in Kapitel 3 erfolgen. Ein Beispiel soll andeuten, daß auch ohne das Vorhandensein geschlossener Zeitlinien räumliche Wirkungen entstehen. Im Roman "Noce $i$ Dnie" von $M$. Dąbrowska ist es gerade der bemußte und systematisch durchgehaltene Verzicht auf die zeitliche Dichte der figuralen Handlungsabläufe, die das räumliche Erleben (nicht des bühnenmäBigen Schauplatzes, sondern der geschichtlichen Epoche) zu einem kontinuierlichen, zeitlich dichten Erlebnis werden läBt. 
Zielinskis Ausgangspunkt kann man so interpretieren, daB es eine besondere Fähigkeit sei, zwei Vorgänge zugleich zu sehen, und daB das darauf beruhende Erlebnis ein Raumerlebnis sei. Dabei betrachtet man zwei Zeichen, die notwendigerweise nebeneinander stehen, als nicht mehr zu dieser Erlebniskategorie gehörig, ohne der von der Psychologie nachgewiesenen Tatsache Rechnung zu tragen, daß der Punkt auf der durch psychisches Erleben festgelegten Zeitlinie durchaus nicht ausdehnungslos ist. Also ist der Moment als der Zeitraum, in dem zwei sprachliche Zeichen aufgenommen werden, zu einem einheitlichen, geschlossenen Augenblickserlebnis verschmelzbar. Deutlich tritt dies beispielsweise bei den Augenblicksbildern in Iwaszkiewicz' Novelle "Ułyn nad Utratq" zutage, wo etwa Farbtöne zu solchen Momentanerlebnissen zusammenwachsen. Hier finden sich auch Wörter wie "Mond" und "abgeschnittener Fingernagel" in solcher Nähe zusammen, daß eine einheitliche Erlebnisqualität entsteht, in diesem Fall die momentane Impression der Unsicherheit und Irrealität der Konsistanz des Wahrnehmungsraums. Die beiden Ausdrücke murden vom Autor auf dem Wege des Vergleichs gefunden, doch steht die nötige Zeit, die beiden Vergleichsebenen zu realisieren, dem Leser nicht zur Verfügung.

Die Auseinandersetzung mit den Gedanken Lessings und Zielinskis ergibt nicht, daB diese falsch sind, sondern daB sie nur einen Aspekt der Sprache und damit des Raumes und des Kunstwerkes berühren und so für eine strukturelle Betrachtungsweise nicht ausreichen. Ein ähnliches Argument kann man auch gegen D. Frey ins Feld führen. Der Grundgedanke seines Werkes 1 ist die Gegenüberstellung von simultaner und sukzessiver Vorstellungsweise; in der sprachlichen Darstellung "muß die sinnliche Vorstellung in das begriffliche Medium der Sprache übergeführt werden, d.h. das einheitliche Vorstellungsbild der Einzelvorstellungen, die begrifflich in der Sprache faßbar sind, zerlegt und aus ihnen zusammengesetzt werden. Wenn ich sage: "Ein roter Mantel fällt ihm von den Schultern", so setzt sich diese Gesamtrorstellung,

1 D. Frey: Gotik und Renaissance als Grundlagen der modernen Weltanschauung, Augsburg 1929. 
die damit gegeben ist, aus den Dingvorstellungen Mantel und Schulter, der Farbvorstellung rot und der Bewegungsvorstellung Fallen zusammen. Will ich die für die Beschreibung notwendigen Worte und Wortbildungen finden, so muB schon im Aufbau der Gesamtvorstellung eine Umwandlung vor sich gehen. Jode Beschreibung ist gekennzeichnet durch den Versuch, die innere Konstitution der Vorstellung so umzugestalten, daB sie durch die gegebenen oder zu bildenden Sprachformen ausdrückbar wird." 1

Auch dieses Beispiel kann stellvertretend stehen für eine weit verbreitete Betrachtungsweise; der Grundgedanke ist, daß Räumliches in Zeitliches aufgeteilt, zerspalten, übertragen oder verwandelt werden muß, wenn es mit einem zeitgebundenen Medium ausgedrückt werden soll. Die Sukzessivität der Worte und Wortverbindungen betrifft wohl ihre zeichenmäBige Wirkungsform, nicht aber ihre Funktion als Symbole. So ist die "Umwandlung" nicht notwendige Bedingung für den Gebrauch des sprachlichen Mediums und damit eine Beschränkung, sondern kann als Technik der Gestaltung zur Anwendung kommen. Und andererseits muß ja auch ein zeitlicher Vorgang in Einzelvorstellungen aufgelöst werden, wodurch er seine Stellung als bevorzugtes Objekt sprachlichen Gestaltens verliert. Wie aber die Zeit als Hintereinander und als Bewegung wahrgenommen und so auch gestaltet wird, ist auch die Wahrnehmung und das Erlebnis des Raumes als psychisches Paktum ein zeitlich ausgedehnter Akt.

Die Zuordnung von Zeichen und Komponenten eines Komplexes und speziell eines räumlich angeordneten Komplexes ist also der Ausgangspunkt. Grundlage ist die Erkenntnis, daß das sprachliche Zeichen nicht aus der Sprechsituation herausgelöst werden darf, wenn es seine Bedeutung erfüllen und seine Funktion erkannt werden soll.

Für diese Situation können räumliche Relationen relevant sein oder nicht; am Beispiel des Erzählens sollen die Möglichkeiten und Relevanzgrade räumlicher Bestimmung für diese Situation untersucht werden, wobei $\mathrm{K}$. Bühlers 2 Ausgangspunkt, sprachliches

1 A.a.0., s. 162 f.

2 K. Bühler: Sprachtheorie. Die Darstellungsfunktion der Sprache, Stuttgart $1965^{2}$. 
Darstellen sei die Befreiung der Gegenstände aus ihrer Situationsgebundenheit, ergänzt wird durch den Satz: Erzählen ist der Aufbau einer durchgehend oder zeitlich begrenzt wirksamen Situation. Da aber auch beim Erzählen die Deixis, d.h. die Ausnützung des um den Sprecher zentrierten Bezugssystems, Verwendung finden kann, zitiere ich $\mathrm{K}$. Bühlers Formulierung dieser Gegebenheit: "Jedenfalls aber verstehen wir allgemein, das das Bedürfnis, den Darstellungsgehalt einer Rede frei zu machen vom aktuellen Zeigfeld, in der erzählenden Rede aufkommt. Man kann sich im großen Entwicklungsgang der Menschensprache Einklassensysteme deiktischer Rufe als das erste vorstellen. Dann aber kam einmal das Bedürfnis, Abwesendes einzubeziehen und das hieB, die Außerungen von der Situationsgebundenheit zu befreien. Die Mittel dazu sind für zwei Hauptfälle in der Sprache, die wir selbst sprechen, angegeben und psychologisch beschrieben. Die Enthebung einer sprachlichen AuBerung aus dem Zeigfeld der demonstratio ad oculos beginnt in unserer eigenen Sprache entweder raum-zeitlich, indem eine nennende Versetzung anstelle der primär ungeformten, weil mitenthaltenen hier-jetzt-Deixis eingeführt wird (Impersonalia). Oder sie beginnt an der primär ebenso ungeformten, weil mitgegebenen ich-Deixis (Verbalsatz)."1

An das Erzählwerk ist also zunächst die Frage zu richten, wie der Raum mit den Mitteln der Sprache behandelt wird, wie er im Erzählzusammenhang fungiert und welcher Aspekt des Gesamtwerkes seiner Erfassung dient. Dann aber wird als gleichwertiges Problem die Frage der Interpretation zu lösen sein. In der "Lalka" von Prus z.B. erfolgt das Eingehen des Autors auf den Raum als Schauplatz in traditioneller Weise und legt keinerlei Eigenart des Werkes frei; erst bei der Suche nach den Integrationsmomenten, in welchen spezifisch Heterogenes und Widersprüchliches vereinigt wird, ist die Kategorie des Raumes für die Analyse notwendig.

1 A. a. O. S. 279 f. 
2. Die Zuordnung von sprachlichem Zeichen und räumlicher Gegebenheit

Das folgende Kapitel dient der Klärung und Abgrenzung des eigentlichen Objektes der literarischen Raumanalyse. Die nächstliegende Frage lautet: Welche Zeichen dienen der Bezeichnung räumlicher Gegebenheiten? Die Antwort auf diese Frage kann in einer Aufzählung lexikalischer Einheiten resultieren, die auch außerhalb des Erzählkontextes ihre raumbezogenen Vertreterfunktionen ausüben. Es handelt sich also um ein sprachwissenschaftliches, genau genommen semantisches Problem. $1 \mathrm{Da}$ dieses nicht im Bereich der vorliegenden Arbeit liegt, begnüge ich mich mit der Unterscheidung von Zeichen für raumfüllende Gegenstände, räumliche Relationen und für den Raum als Ganzes. Die Bedeutung dieser Unterscheidung zeigt sich bei der Analyse von Andrzejewskis Roman "Bramy Raju", in dem jeder Klasse der oben genannten Zeichen deutlich trennbare Aufgaben zufallen. Es läßt sich dort nämlich eine Schicht von Wörtern feststellen, die dem historischen Schauplatz, einer Landschaft in Frankreich, entstammen. Das sind die Wörter: Wiese, Mühle, Dorf, Weide, Hütte usw.; ferner: Schloß, Kathedrale, Purpurmantel, Schwert, Pfeil usw. aus dem Bereich des höfischen Lebens, und drittens die Einzelheiten, die der Charakterisiemung des Weges dienen, Naturgegebenheiten wie: Urwald, Dickicht, Pfad usw. Diese Gegenstände werden erwartungsgemäB durch eine Anzahl von Positionsangaben, hauptsächlich Präpositionen, in eine räumliche Beziehung zueinander gesetzt. Diese Positionsangaben spielen zahlenmäBig eine große Rolle. Ihre Funktion wird dadurch strukturell prädominant vor den gegenstandsbezeichnenden Wörtern, daß sie in gleicher Weise auch bei anderen, unräumlichen Gegebenheiten auftauchen und auch in den Bereichen: Liebe, religiöses Wunschdenken usw., räumliche Relationen schaffen. Sie vertreten also nicht nur räumliche Relationen, sie stellen diese auch in origineller Weise her.

Wenn man sich vom rein sprachlichen Bereich weg- und dem Erzäh-

1 Vgl.: G. Altmann, Z. Dömötör, A. Riska: The partition of time in Nimboran, in: Beiträge zu Iinguistik und Informationsverarbeitung 12 (1967), S. 56-71. 
len zuwendet, kann die Hethode des Auszählens von Wörtern einen interessanten und wesentlichen Weg zeigen. Man kann die mit einem Erzählabschnitt verbundenen raumbezogenen Wörter zählen und dann den Prozentsatz dieser Zeichen im Verhältnis zur Gesamtzahl errechnen, wobei die Frage der Verteilung, der Häufung, der Zuund Abnahme oder sonstiger Proportionen besonders aufschluBreich ist. Das strukturell bedeutsamste Ergebnis dieser Verfahrensweise ist folgendes: Beschränkt man sich auf den Anfang, die ersten Sätze des betreffenden Erzählabschnlttes, und geht davon aus, daß der Leser ursprünglich vor einer tabula rasa steht, d.h. keinen Raum anschaulich vor sich hat, so bietet eine verhältnismäBig kleine $\mathrm{Zahl}$ von Zeichen eine sehr viel größere Zahl von räumlichen Elementen. Selbst wenn dem Raum an diesem Anfang keine gesonderte Nennung oder gar Beschreibung zukommt, erfolgt bereits an dieser Stelle die entscheidende Versetzung in den Raum, z.B. an den Ort der Handlung. Betrachtet man dagegen die Fülle der raumbezogenen Zeichen, die mit einer abgeschlossenen Erzählung verbunden bzw. gegeben sind, so ist die Zahl der Einzelinformationen, die der Leser durch sie über den Raum erhält, sehr viel geringer. Man kann, gleichgültig wie sich dieses Verhältnis im Einzelfall gestaltet, von einer zeichenmäßigen Redundanz sprechen, da eine rein auf den Raum bezogene und konzentrierte Darstellungsweise mit einer geringeren Zahl von Zeichen ausgekommen พäre.

Das Ergebnis dieser Gegenüberstellung von Aussagen über den Raum im Erzählkontext, bei denen einerseits durch eine kleine Zahl zeichenmäBiger Mittel ein komplexer Raum anschaulich realisiert wird, und der Redundanz von Zeichen andererseits, die einen tberfluß an Wörtern im Verhältnis zur raumbezogenen Aussage liefert, kann für die Werkanalyse in zweifacher Weise fruchtbar werden. Es zeigt sich erstens, daß eine rein zahlenmäBige Korrespondenz von Zeichen und räumlichen Elementen nicht generell und normativ für das Erzählen festgelegt werden kann. Erst der Blick auf das ganze Erzählwerk kann zeigen, wie raumbezogene Zeichen oder Erzählphasen sich einordnen, wobei der Blickwinkel so gewählt werden muß, daß Aussagen über den Raum nicht isoliert neben Erzählelementen stehen, die keine Information über den Raum beinhalten; 
vielmehr schließen sich beide Elemente zu einem Ganzen zusammen, das seinerseits wieder in bestimmtem Maße raumgebunden ist. Strukturelle Betrachtungsweise bedeutet, daß jedes dieser Elemente, seien es Zeichen oder Erzählphasen, gleichberechtigt ist und dementsprechend, auch wenn es nichts über den Raum aussagt, für diesen nicht irrelevant ist. Neben das Prinzip der Redundanz muB also, um einen weiteren Ausdruck der Informationstheorie heranzuziehen 1, das der optimalen Inkodierung gestellt werden, wonach kein Zeichen überflüssig ist.

Der zweite Punkt betrifft die Integration der raumbezogenen Elemente in das Gesamtwerk. Daß das Erlebnis oder die Wirkung des Raumes abhängig ist oder mitbestimmt wird von der entsprechenden Stellung im Erzählablauf, sei es zeit- oder kontextbedingt, teilt dieser mit anderen Gegebenheiten, etwa Charakteren oder Handlungseinheiten. Hier geht es aber un die spezifischen Eigenheiten des Raumes. Daß am Anfang der Erzählung wenige Zeichen viel über den Raum aussagen, im Weiterverlauf die Aussagekraft abnimmt, die Redundanz also zunimmt, ist in keinem Werk in Form einer gleichmäßig fortschreitenden Entwicklung verwirklicht. Der Grundsatz, daB Information über den Raum nur nötig ist für jemanden, der sich nicht in ihm befindet bzw. für den er neu ist, wirft auf die Verteilung der raumbezogenen Zeichen und damit auf den Aufbau des Erzählwerkes ein klärendes Licht. Dazu seien einige Beobachtungen aus den Anwendungsbeispielen des zweiten Teiles dieser Arbeit vorweggenommen.

Die Raumfiktion in "Quo vadis" erfolgt nach dem Prinzip, daß Autor und Leser als in diesem Raum anwesend vorausgesetzt werden. Der Autor nennt nur das, was im Raum an Besonderheiten, auffallenden und ausgefallenen Gegenständen vorhanden ist. Die normalen räumlichen Verhältnisse, die den räumlichen Gesamtaufbau bestimmen, bleiben unberücksichtigt oder erscheinen nur insoweit im Erzählzusammenhang, als eine lateinische Benennung möglich ist. Die Rolle des Autors ist die des Kommentators, für den sich eine genaue Angabe der Position für die erwäbnten Details erübrigt, da seine Zuhörer ja neben ihm stehen. Die Zeichen für räum-

1 Siehe R. Gunzenhäuser: Asthetisches MaB und Ësthetische Information. Quickborn bei Hamburg 1962, S. $83 \mathrm{ff}$. 
liche Details haben ihre Angemessenheit gegenüber dem darzustellenden Objekt nicht aufgrund eines auktoralen Auswahlprozesses, der eine möglichst genaue Aussage anstrebt, sondern durch die begriffliche Distanz, welche von einem anderen Sektor, dem der historischen Wissenschaft, übernommen ist.

Auch in Prus' "Lalka" wird eine genaue Kenntnis des Schauplatzes, nämlich der Stadt Warschau, beim Leser vorausgesetzt. Räumliche Bilder oder andere Konzentrationen raumbezogener Zeichen sind immer wieder in den Erzählverlauf eingefügt, sie präzisieren aber nicht den Schauplatz, sondern sie beleuchten einen ganz bestimmten psychischen Zustand der Figuren, und dieser Zustand ist im jeweiligen Fall neu und problematisch geworden. Diese Abschnitte sagen auch über den Raum, wie das Paris-Kapitel deutlich zeigt, nur verhältnismäBig Unklares aus, charakterisieren aber den Standpunkt der $F_{i g u r}$ mit Hilfe der eigentümlichen sprachlichen Perspektive.

Auch Żeromskie Raumschilderungen sind nie ausschlieblich auf das Lokal als Selbstzweck ausgerichtet. Die diesbezüglichen Erzählabschnitte zerfallen deutlich in zwei aufeinanderfolgende Teile. Der erste zeigt jeweils den Wahmehmungsraum in szenischer Form. Der zweite ist eine Interpretation dieses Raumes im sozialkritischen Sinne. Nur im Zusammenspiel beider Schichten entsteht die raumbezogene Erzählphase.

In Iwaszkiewicz' Novelle "Młyn nad Utratg̨" dienen die der räumlichen Sphäre entstammenden Zeichen dem Aufbau einer Vergleichsebene mit veranschaulichender Wirkung. Charakteristisch für die Auswahl von Zeichen für den Raum ist in diesem Werk das übergangslose Nebeneinander von Gegebenheiten verschiedenster Kategorien. Das unmittelbare Nebeneinander dieser Zeichen wirkt nicht durch Gegensätzlichkeit und Antithetik, sondern durch den $\mathrm{Zwischen.}$ zustand, der dadurch entsteht, daB keine dieser beiden Kategorien eine feste Ausgangsbasis ist, zu der lediglich kontrastive Elemente hinzugefügt werden. Dies ist der Ausdruck für einen Zwischenzustand der Figuren, etwa inre existentielle Unsicherheit usw. Hier kommt als ein weiteres Element dazu, daB die wörter nicht nur Zeichen im Sinne von Vertretern sind, sondern auch 
Ausdruckswert besitzen. Bei der Darstellung des Raumes im Erzählzusammenhang ist es ein wesentlicher Grundsatz, daß nicht das damit verbundene Zeichenmaterial in Wörter der einen und Wörter der anderen Kategorie zerfällt, sondern das gleiche Wort einmal in der einen, einmal in der anderen Zuordnungsform auftritt. Zwar gibt es Zeichen, die nach einer bestimmten Richtung tendieren und diese Funktion nahelegen. Aber das Beispiel von Bruno Schulz zeigt, daß gerade die Termini der wissenschaftichen Beschreibung, deren einzige Berechtigung es ist, ein fest umrissenes Bedeutungsfeld zu haben, in der entgegengesetzten Form angewendet und damit erlebt werden können. An dieser Stelle weise ich auf die von ihm bevorzugte Verwendung von Fremdwörtern hin (Figuration, Konstellation usw.), die Schulz' Raumfiktion prägen und ihr ihre eigentümliche Gestalt verleihen.

Wendet man nun den Blick auf größere sprachliche Einheiten, also Zeichen-bzw. Satzkombinationen, die ich im Hinblick auf meinen Untersuchungsgegenstand Erzählphasen nenne, so kommen zwei weitere Probleme hinzu: Das Verhältnis des einzelnen räumlichen Gegenstandes zum Gesamtraum sowie die Funktion des einzelnen Zeichens als Vertreter für ein räumliches Element im Rahmen einer grammatisch geordneten Zeichenfolge.

Das Merkmal des Darstellens ist bei $\mathrm{K}$. Bühler die Situationsentbindbarkeit. Das bedeutet: Ein Gegenstand, der mit einer Situation verbunden ist, wird aus dieser herausgelöst und an anderer Stelle, außerhalb dieser Situation, genannt und damit wieder anschaulich realisiert. Ich kann also mit einem Partner von einem bestimmten Gegenstand sprechen, ohne daß dieser vor uns liegt und unseren Sinnen zugänglich ist. Für den Bereich des Erzählens muß dieser einfache Vorgang um eine entscheidende Komponente erweitert werden: Nicht nur ein Gegenstand wird aus der Abwesenheit herbeigeholt, die ganze Situation ist transponierbar; sie kann durch einen Erzählakt anschaulich realisiert werden, ohne daß auch nur eine ihrer Komponenten den Sinnen hic et nunc zugänglich ist und ohne daß, was für den Raum besonders wichtig ist, alle Komponenten mit adäquaten Zeichen versehen werden. Das ist die Grundlage der Raumdarstellung. 
Die Raumdarstellung muß, sobald man sie im Erzählzusammenhang und damit im strukturellen Rahmen untersucht, unabhängig von der auktoralen Intention betrachtet werden. Denn neben Werken, in denen die Darstellung des Schauplatzes Ziel des Autors und folglich seine Technik Gegenstand der Untersuchung ist, finden sich Werke, in welchen die Spuren des Eingehens auf den Raum minimal sind und doch ein äuBerst intensives und farbiges Bild vom Schauplatz beim Leser entsteht. Zwischen diesen beiden Polen liegt eine mannigfaltige Reihe von Fällen, in denen der Raum nur detailhaft und in Ausschnitten vom Autor festgelegt ist, die Ergänzung und Verbindung der Teile aber der Illusionsfähigkeit des Lesers überlassen bleibt.

Die Möglichkeiten, mit einer beschränkten Anzahl von raumbezogenen Wörtern einen äußerst komplexen Raum aufzubauen, wie es das Wesen der Raumdarstellung ist, sind sehr verschiedenartig. Für das Problem des Raumes im Erzählwerk werden diese Lösungen, wie die Anwendungsbeispiele unten zeigen, mengenmäßig das gröBte Kontingent stellen. Geht man aber von den Beispielen aus, wo im Werk vom unreflektierten Leserstandpunkt her betrachtet ein Raum empirisch gefunden wird, und fragt, welche sprachlichen Gegebenheiten dafür verantwortlich sind, so wird man Fälle finden, in denen einem vollständigen Raum kein einziges Zeichen entspricht, dessen Bedeutungsbereich auf ein räumliches Element schließen läBt. Vielmehr ist dieser Raum fortschreitend mit dem Erzählvorgang entstanden und liegt erst mit dem abgeschlossenen Erzählvorgang als Ganzes vor. Erst die Vollständigkeit des räumlich irrelevanten sprachlichen Ereignisses resultiert in einem vollständigen Raum, wobei die Art des Erlebnisses und nicht die Zahl der Komponenten Kriterium der Vollstündigkeit ist, oder in der räumlichen Anordnung von Elementen, die, jedes für sich betrachtet, nicht räumlich sind. Ein gutes Beispiel dafür bietet, wie ich weiter unten zeige, der Roman "Noce i Dnie" der Maria Dąbrowska, wo der gewaltige Raum einer historischen Epoche dem Ieser anschaulich vor Augen steht, ohne daB er expressis verbis berührt wird. Diese Erscheinung nenne ich Raumgenese. Während das Erzählen die Darstellung des Raumes mit dem Sprachgebrauch im weiteren Sinne teilt, ist die werkimmanente Raumgenese dem in 
sich geschlossenen, künstlerischen Erzählen in Form von Novelle bzw. Roman vorbehalten.

Die Grundlage für die Raumgenese ist wiederum in einer Eigenart des Wortes und seiner Kombinationsmöglichkeit angelegt. Diese Eigenschaft des sprachlichen Zeichens, die über die Funktion der Vertretung hinausgeht, charakterisiert $\mathrm{K}$. Bühler folgendermaßen: "Wenn nun hic et nunc ein Konkretum als Vertreter fungiert, so kann stets die Frage erhoben werden, kraft welcher Eigenschaften es die Vertretung erhielt und in die Vertretung eingeht, sie erfüllt. Es muß also stets eine zwiefache Bestimmung dieses Konkretums möglich sein, von denen die eine absieht von der Punktion des Vertretenden Vertreter zu sein, um es so, um es als das zu bestimmen, was es für sich ist oder wäre. Die zweite Auffassung dagegen sucht und findet an inm diejenigen Eigenschaften, an welche die Vertretung gebunden ist. Im Falle des Zeichenseins sind es immer nur abstrakte Momente, kraft derer und mit denen das Konkretum "als" Zeichen fungiert. Ich habe diesen sprachtheoretisch grundlegenden Tatbestand als das Prinzip der abstraktiven Relevanz bezeichnet und am Unterschied von Phonetik und Phonologie erläutert." 1 Diese Aufspaltung des Zeichens und seiner Funktion in eine abstrakte und eine konkrete Komponente, und zwar jedes einzelnen Zeichens, ist für das Erzählwerk von größter Bedeutung. Sie verschiebt das Gewicht von der eindeutigen und nur in eine Richtung weisenden Zuordnung von Vertreter und Vertretenem auf die konkrete, vom Bezeichneten unabhängige Existenz des Zeichens und seine Fähigkeit, einen spezifischen Realitätsbereich zu bilden. Die mit einem Erzählwerk verbundene Masse von Zeichen bietet damit nicht eine ebenso große Masse von bezeichneten Gegenständen, sondern ist zumindest teilweise an der Genese eines solchen Bereiches beteiligt. Ein kleines Beispiel für die Raumgenese, in welcher kein räumliches Element auf der sprachlichen Seite der Entstehung eines ganz spezifischen Raumes entspricht, findet man in der Erzähltechnik Eichendorffs. R. Alewyn analysiert einen Satz, der für

1 A.a.0. S. 40 . 
dessen Technik der Landschaftsdarstellung 1 als repräsentativ angesehen werden kann, nämlich: "Alle Vögel sangen in der schönen Einsamkeit", folgendermaßen: "Mit diesem Ort hat es nun eine besondere Bewandtnis: in der schönen Einsamkeit - nehmen wir vorweg, daß das Adjektiv wieder reichlich nichtssagend ist. Es lieBe sich unschwer mit dem "herrlichst" des Sommermorgens vertauschen. Daß Eichendorff sich mit farblosen Adjektiven wie diesen meist begnügt, ist mehrfach bemerkt worden. Aber könnte es in diesem Falle fehlen? "Alle Vögel sangen in der Einsamkeit"? Es entstünde sogleich eine Unbehaglichkeit, weil damit den Vögeln, und gar "allen Vögeln" etwas zugeschrieben zu werden scheint, was ihnen nicht zukommt: Einsamkeit. Das Attribut "schön" erst stellt klar, daß mit "Einsamkeit" hier kein Zustand gemeint ist, sondern ein Ort und daß somit "in" eine streng lokale Bedeutung hat." 2

Wenn das Ergebnis Alewyns hier lautet: das Abstraktum ist ein Teil der Landschaft, so kann es sich dabei nur um eine Charakteristik der Wortart handeln. Daß nämlich die Einsamkeit in Verbindung mit dem Attribut "schön" auftaucht und dieses dem Zeichen zugeordnet ist und nicht dem bezeichneten räumlichen Gegenstand, weist darauf hin, daB das Zeichen selber etwas konkret Situationsbildendes darstellt. Es wirkt in diesem Zusammenhang nicht als ein Vertreter für etwas, sondern ist selber ein wahrnehmbares, konstituierendes Moment einer Situation, welche im Augenblick des Leseaktes im Entstehen begriffen ist.

Die Zuordnung von Zeichen und räumlicher Gegebenheit findet auf drei fortschreitend darstellbaren Stufen statt:

1. Die bloße Vertretung jeweils eines räumlichen Elementes durch ein sprachliches Zeichen;

2. Die Darstellung des Raumes unter Ausnützung der sprachlichen Fähigkeit, Situationen zu übertragen und vorauszusetzen;

3. Die Raumgenese, wobei Fortschreiten und Abgeschlossenheit des Erzählvorgangs mit Entstehung und Vollständigkeit des Raumes korrespondieren.

1 R. Alewyn: Eine Landschaft Eichendorffs, in: Euphorium 51 (1957), S. 47-60.

2 A.a.0., S. 49. 
Mit einem Erzählwerk ist eine Pülle von zeitlichen Relationen und Größen gegeben. Es ist eine Aufgabe der Literaturwissenschaft, diese Gegebenheiten zu differenzieren und dann wieder zu koordinjeren, um so den Kunstcharakter des betreffenden Werkes näher zu bestimmen. Andrzejewskis Roman "Bramy raju" ist ein Beispiel dafür, welche Fülle von Möglichkeiten es gibt, zeitliche Muster dem Erzählwerk zugrunde zu legen. Die Skala reicht hier von der historischen Epoche und dem chronologischen Hintereinander bis zur Wiederholung bestimmter Bilder oder einzelner Symbole und Formeln. Es ist äußerst schwierig, diese Differenzierung so vorzunehmen, daB damit die relevanten Schichten des Werkes erhellt werden und nicht eine unnötige Problematisierung entsteht. Spricht man bei diesem Werk z.B. von der einschnittbildenden Funktion des häufig auftretenden Zeigwortes "plötzlich", so muB man herausfinden, auf welcher Ebene tatsächlich Einschnitte durchgeführt sind. Dabei kann man feststellen, daß es zunächst der Erlebnisverlauf der Figuren ist, der in eine dramatische Folge von Phasen unterteilt wird. Aber dieser Folge, so wird unten gezeigt, fehlt es an dramatischer und psychologisch motivierter Konsequenz. Sieht man aber dieses Wort "plötzlich" in Verbindung mit der Menge anderer deiktischer Partikeln sowohl zeitlicher als auch räumlicher Art, so zeigt sich eine Sukzession sui generis, die abstrakt und unabhängig von der beschriebenen Realität existiert und die Besonderheit der in diesem Werk realisierten Struktur ausmacht. Daraus kann man für die Analyse den Grundsatz ableiten, daß die bloße Unterscheidung von Zeitpunkten und zeitlichen Einheiten nutzlos ist, wenn man nicht die dazugehörige Zeitlinie findet, auf der diese Gegebenheiten lokalisierbar sind. Findet man z.B. eine Wiederholungsfigur leitmotivischer Art, mu man untersuchen, wer der Träger dieser Figur, dessen Zeitgebundenheit oder Zeiterleben diese Elemente erst zu einer Figur macht, überhaupt ist. Ist es der Erzähler mit seinem reflektierenden Bewußtsein, die mit dramatischer Konsequenz ablaufende Handlung, oder ist es eine andere Schicht, die für die Struktur des betreffenden Werkes auf diese Weise relevant wird? Die Aufdeckung von zeitlichen 
Relationen hat für die Analyse den Nutzen, daß man bei der Suche nach der zugrundeliegenden Zeitlinie, auch wenn es sich bei dieser um eine HilfsgröBe oder ein veranschaulichendes Bild handelt, die Existenz und die Relevanz von Schichten eruieren kann. Yan sieht z.B. In Iwaszkiewicz' Erzählung "MYyn nad Utratq̨", daB die dramatische Konsequenz als gestalthafte und ästhetisch wirksame Qualität von der Handlung, dem Konflikt und der dramatischen Entwcklung der Figuren weggenommen und in die Folge momentaner Raumbilder verlegt ist. Ohne die spezielle Analgse mit Hilfe zeitlicher und räumlicher Kategorien könnte man zwar auch eine Folge von sich verändernden figuralen Seelenzuständen herausfinden; doch ist der SchluB des Werkes, der Todesmoment, mit einer psychologisch orientierten Analyse nicht als konsequenter Fndpunkt zu erklären.

Bisher hatten wir es nur mit einer vorgegebenen Menge zeitlicher Gegebenheiten, Relationen und Einheiten zu tun, aus welcher die Analyse die relevanten auszuwählen hat. Eine andere Problematik muß aber neben diese gestellt werden, ja sie muB dieser vorausgehen. Dies ist die Frage: Wie entstehen in einem Erzählwerk zeitliche Einheiten und Größen? Das Werk besteht aus einer Fülle von Zeichen, diese haben Jedoch keine zeitlich meBbare, für das Werk relevante GröBe. Sie sind in dieser Ausdehnungslosigkeit zunächst nur Bedeutungsträger, die in Form einer Folge auftreten oder zu einer solchen verbunden sind. Von diesem Standpunkt aus gesehen bedeutet Erzählen das Aneinanderreihen von wörtern, für welche die Angemessenheit gegenüber dem darzustellenden Objekt ausschlaggebend ist. Dieses Verfahren, so hat das 2. Kapitel ergeben, ist nicht im Wesen der Sprache verbindlich angelegt oder als darstellungstechnischer Mangel des sprachlichen Mediums anzusehen; es kann als auktorale Konzeption dienen, die von einem vorliegenden Objekt ausgeht, alle seine Komponenten zu erfassen versucht und erst dann zu erzählen aufhört, wenn der Gegenstand vollständig erfaBt ist. MaBstab für diese Vollständigkeit ist das Objekt. (Natürlich kann auch die Konzeption des Autors diesen Maßstab bilden, dabei liegt aber eine andere Zeitstruktur zugrunde. S.u.) Charakteristisch für diese Art von Zeichensukzession ist die Irrelevanz der Reihenfolge, in der sie dargebo- 
ten werden.

Das Zeichen, das ein Glied dieser Art von Sukzession bildet, erhält seine hervorragende Funktion durch die Bedeutung des Gegenstandes, den es adäquat vertritt. Angemessenheit bedeutet, daB das betreffende Zeichen auch am anderen Ort, außerhalb des jeweiligen Kontextes, stets die gleiche Gegebenheit vertritt. Dieses Zeichen ist ein zeitlich ausdehnungslóses Glied, solange nach ihm gesucht und es überprït wird, solange also das Auswahlprinzip anzusetzen ist und die Adäquatheit den Ausschlas gibt. Erst das nicht adäquate Zeichen drängt nach Weiterführung, ist kontextgebunden und somit konstituierendes Element eines zeitlichen Prozesses.

Adäquatheit kann dabei sowohl objektive Angemessenheit gegenüber dem eindeutig festgelegten Gegenstand bedeuten als auch ein subjektives Erlebnis des Zutreffens und ErfaBtwerdens von weniger deutlich abgegrenzten Realitätsbereichen zur Grundlage haben. Für die Analyse bedeutet das, daB beim Erzählen der Autor nicht unter ein paar möglichen Zelchen das angemessenste auswählt, sondern eine Schicht von Wörtern benützt, für welche Angemessenheit ein Teil des Bedeutungsbereichs ist. B.Schulz verwendet beim Frzählen bevorzugt wissenschaftliche Termini, deren Hauptfunktion ja die adäquate Erfassung eines fest begrenzten Bereiches ist.

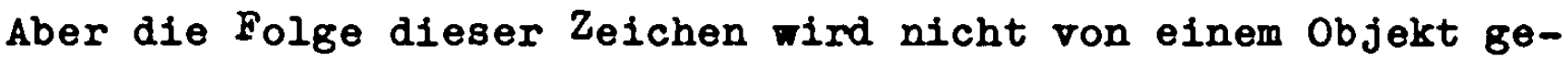
steuert, sondern vom Autor. Bleibt der Leser bei den Objekten, so wird er durch den Verlauf der Sukzession überrascht. Auf diesem Zwischenzustand und der darin begründeten Spannung liegt der ästhetische Effekt von Schulz' Erzählwerken.

Die Fälle, in denen man einen konkreten Raum als Vorlage und Gegenstand sprachlichen Darstellens einwandfrei identifizieren kann, sind in der Erzählliteratur als Sonderfälle zu betrachten. Nur für den Raum, der als ein Objekt vorliegt, gilt folgende Charakteristik: Er konstituiert sich durch die Simultanität aller Komponenten. Seine Wahrnehmung erfolgt, da er sich nicht verändert, momentan, dauert aber von diesem Augenblick an unbegrenzt weiter, eben so lang, bis für jede Komponente ein Zeichen gefunden ist. In welcher Reihenfolge jedoch diese Zeichen dargeboten werden, ist für den Charakter dieses Raumes irrelevant. 
Der Schwerpunkt bei der Analyse dieser Art von Raumbeschreibung wird bei einem bestimmten Wortmaterial liegen. Die wichtige Frage, welches Wort in der Erzählung das Objekt der spezifischen Raumanalyse ist, wird vom Autor in verbindlicher Weise festgelegt, z.B. durch formale Einschnitte oder in Form von topographischen bzw. szenischen Angaben. Fragt man nun nach den auf diese Weise übriggebliebenen Zeichen, so kann man dieses Problem nicht mit einer quantifizierenden Methode lösen, indem man sie als Verbindungsglieder oder Füllwörter ansieht. Um einen Gegensatz für diese erste, ausschlieblich medial gedachte Folge von Zeichen zu finden, übernehme ich eine Begriffsbildung aus der Psychologie.

Diese differenziert die Möglichkeiten der Psyche, sukzessive Erscheinungen wahrzunehmen, und beschreibt, welche Wirkung eine wahrgenommene Sukzession im Zusammenwirken mit den Fähigkeiten der Psyche hervorrufen kann. Hier hebt $\mathrm{K}$. Müller 1 die mediale Sukzession von der Sukzessivgestalt ab. "Die Sukzessivwahrnehmungen können dazu dienen, Gegenstände (statische Gebilde) des anschaulichen Umfeldes $z u$ erfassen und $z u$ erkennen. In diesem Falle sind die Gegenstände eigentliches cognitives Ziel. Die Sukzessionen der Teile dieser Gegenstände treten als phänomenologische Sachverhalte gegenüber den Endgebilden der Sukzession mehr oder weniger zurück. Die Sukzessionen selbst sind dann keine selbständigen und in sich geschlossenen Erlebnisse, sondern sie verweisen in ihrem Ablauf auf ein Gebilde, in dem sie erst ihren AbschluB und damit ihren eigentlichen Sinn finden... Solche Folgen sind überall doft verwirklicht, wo die Gegebenheiten in Wahrnehmungsfeld zu umfangreich und ausgedehnt, wo die Sinnesflächen, der Aufmerksamkeitsumfang oder die Aufnahmekapazität des psychophysischen Organismus $z u$ klein sind, als daB diese Gegebenheiten simultan erfaBt werden könnten." 2 Dem entspräche auf sprachlichem Gebiet die Aneinanderreihung von Bedeutungseinheiten zur Erfassung eines vorgegebenen Raumes. Dabei ist aber nicht die

$1 \mathrm{~K}$. Müller: Der Aufbau figural optischer Phänomene bei sukzessiver Reizung, Frankfurt 1963.

2 A.a.0., S. 31 f. 
Unzulänglichkeit der Aufnahmekapazität notwendige Bedingung, sondern der Wunsch des Autors nach Verdeutlichung und Verständniserleichterung.

K. Müller fährt fort: "Im Gegensatz zu den medialen Sukzessionen stehen die Sukzessivgestalten. Eine Melodie - als Paradigma einer Sukzessivgestalt - erfüllt sich nicht primär in einem Endgebilde, sondern das Erlebnis einer Melodie konstituiert sich unmittelbar in der Dynamik der Tonsukzession selbst.[..] Der Sinngehalt einer Melodie und ihr ästhetischer Wert zeigt sich nicht - zumindest nicht ausschlieblich in einem simultanen Endgebilde, sondern in den spezifischen Verlaufsqualitäten der Tonfolge selbst, zeigt sich in der unmittelbar anschaulichen Beziehung der Sukzessionsglieder[.] Bei Sukzessivgestalten[...] ist die Zeitstruktur (und damit auch die zeitliche Reihenfolge der Sukzessionsglieder) sehr wichtig. Man kann sogar sagen, eine Sukzessivgestalt konstituiere sich in der Hauptsache aufgrund der Zeitstruktur, deren Veränderung wesentlich die Verlaufseigenschaften und damit das Gesamterlebnis der Folge beeinfluBt."1

Beschränkt man sich bei der literarischen Analyse auf diese Gegenüberstellung, so tritt eine wesentliche Eigenschaft des erzählenden Sprachgebrauchs zutage: Eine Zeichenfolge der ersten, rein medialen Art, die vom Blick auf das Endergebnis gesteuert wird, wird dieses Gebilde wohl erzielen; aber es wird beim Leser auch durchaus ein spezifisches Folgeerlebnis geben, welches mit dem - in unserem Falle räumlichen - Endergebnis nichts zu tun haben muß. Ein Autor kann beispielsweise eine pedantisch genaue und detaillierte Raumbeschreibung liefern, an deren Ende das von ihm angestrebte simultane Endgebilde schauplatzmäßiger Art vorliegt. Gleichzeitig ist aber, während des Lesevorgangs, beim Leser ein sehr lebendiger Eindruck von diesem Autor entstanden. Dieser hat z.B. durch seine Art, die Dinge zu sehen und zu nennen, einen Einblick in seine Persönlichkeit oder seine Wirklichkeitsauffassung gegeben, wie sie bei einer direkten Beschreibung nie entstanden wären. Es ist klar, daß diese Eigenart der

1 A. $.0 .0 .$, s. 32 . 
sprachlichen Zeichenfolge als Erzähltechnik in mannigfacher Weise ausgenützt wurde; ein kleines, aber merkmalhaftes Beispiel, wie unwichtig das simultane Endgebilde bei einer Raumbeschreibung werden kann, zitiere ich aus G.A. Bürgers "Münchhausen": "Stellen Sie sich, meine Herren, das Schreckliche meiner Lage vor! Hinter mir der Löwe, vor mir der Krokodil, zu meiner Linken ein reißender Strom, zu meiner Rechten ein Abgrund, in dem, wie ich nachher hörte, die giftigsten Schlangen sich aufhielten." 1 Eine genaue Rekonstruktion dieses gefährlichen Ortes läuft nicht nur der Intention des Erzählers entgegen, sie ist auch aufgrund der Angaben ganz unmöglich, da ein FluB und ein Abgrund nicht in solcher Nähe möglich sind und auch die giftigen Schlangen im erzählten Augenblick noch gar nicht als Situationskomponente vorliegen.

Für die Zeitstruktur des Erzählwerkes muß also sowohl die Erzählphase als auch das Zeichen als Glied einer Folge angesetzt werden, ohne daB ersteres auf letzteres reduziert werden könnte. Es kann nicht eine der beiden Sukzessionsarten als Wesen der sprachlichen Folge angesehen werden, vielmehr ist diese gerade durch die Uberlagerung und die Korrespondenz beider Folgeweisen charakterisiert. Wie das Erlebnis einer Beschleunigung durch diese Uberlagerung erzielt wird, untersucht H. Cruickshank 2 am Beispiel des Romans in Tagebuchform "La Symphonie Pastorale" von A. Gide. "Three distinct time series are consequently involved. These may be described as the diarist's past (equivalent to the present of the characters whose story he tells), the diarist's present and the reader's present. In other words, the events recorded by the pastor occur at one temporal level. The actual writing of the diary itself takes place at a different temporal level." 3

Diese Technik der Zeitgestaltung, welche der Autor "compression of time" und "technique of condensation" nennt, wird mit folgen-

1 G.A. Bürger: Wunderbare Reisen zu Wasser und zu Lande, Leipzig O.J.

2 J. Cruickshank: Gide's treatment of time in "La Symphonie Pastorale", in: Essays in Criticism 7 (1957), S. 134-143.

3 A.a.0., S. 135 . 
den Mitteln erreicht: Im ersten Teil des Romans kommen 81 Seiten auf einen Monat der dargestellten Zeit, im zweiten 45. Im ersten sind die 81 Seiten in 7 Tagebucheintragungen aufgeteilt, im zweiten sind es 45 Seiten und 3 Eintragungen. Der Abstand von Schreibgegenwart und beschriebener Gegenwart ist am Anfang einige Jahre, am Schluß gleich Null. "In the exclamation: ah! les voicil the past and the present of the diary have finally merged into one." 1 Die Erzählphasen werden also verlängert, die Anzahl der Zeichen, die auf einen Abschnitt des erzählten Vorgangs treffen, wird verringert. Die Wirkung dieser Technik ist: "The reader is not merely told that the pastor is tense and bewildered, and that events are more quickly. He really experiences this tension, this bewilderment and this quickened pace." 2 Die Differenzierung zweier verschiedener Sukzessionsarten bei einer Folge sprachlicher Zeichen, die auch K. Bühlers Gegenüberstellung von Sprechakt und Sprachwerk zugrundeliegt 3, zeitigt auf dem Gebiet des Erzählens dieses Ergebnis: Jeder Erzählvorgang ist als mediale Sukzession und als Sukzessirgestalt aufzufassen und trägt die Merkmale beider Sukzessionsarten. Das Ansteuem eines simultanen Endgebildes ist ebenso mit einer Folge von Zeichen, deren Wirkung von der Relevanz des Hintereinander bestimmt wird, verbunden, wie mit dem Sprechakt eine auch über die Dauer der Erzählphase hinausreichende situationsbildende Wirkung verbunden ist. Diese These kann an der Gegenüberstellung 2weier verschiedener Erzähltechniken nachgeprüft werden, nämlich der Häufung und der Wiederholung.

Ein Vertreter des ausschlieBlich medialen Sukzessionscharakters der Sprache ist $W$. Ivins. Er betrachtet als das wesentliche Merkmal des Wortes seine Wiederholbarkeit, wobei sein exakter Bedeutungsbereich in definierbarer Form immer wieder gegeben ist. 4 Die daraus resultierenden Folgen charakterisiert er folgendermaßen: "Also, I believe, the well known notions of substance and attributable qualities can be derived from this operational

1 A.a.0., S. 141 .

2 A.a.0., s. 138.

3 A.a.0., S. 53 .

4 W. Ivins: Prints and visual comunications, London 1953. 
dependence upon exactly repeatable verbal descriptions and definitions - for the very linear order in which words have to be used results in a syntactical time order analysis of qualities that actually are simultaneous and so intermingled and so interrelated that no quality can be removed from one of the bundles of qualities we call objects without changing both it and all the other qualities. After all, a quality is only a quality of a group of other qualities, and if you change anyone of the group they all necessarily change. Whatever the situation may be from the point of view of visual awareness of the kind that have to be used in an art museum the object is a unity that cannot be broken down into separate qualities without becoming merely a collection of abstractions that have only conceptual existence and no actuality. In a funny way words and their necessary linear syntactical order forbid us to describe objects and compel us to use very poor and inadequate lists of theoretical in-gredients in the manner exemplified more concretly by the ordinary cook book recipes." 1

Die Einseitigkeit dieses Aspektes tritt klar zutage, wenn man die Auflösung eines einheitlichen Objektes in verschiedene Qualitäten und die daraus resultierende Verwandlung in ein inadäquates Gebilde nicht als einen darstellungstechnischen Nachteil, sondern als das Wesen des dichterischen Sprachgebrauchs betrachtet. Wie sehr in diesem Zusammenhang mit der verbalen Wiederholung eine spezifisch ästhetische Wirkung erzielt wird, weist H. Meyer an Beispielen der modernen Erzählkunst nach. 2 Er geht zunächst auf die Raumsuggestion als Ziel einiger Autoren des Realismus ein und untersucht dann, wie gerade durch das Vermeiden dieser Illusion die ästhetisch gleichwertigen Strukturfunktionen des Raumes erreicht werden. "Ganz anders steht es um die Raumgestaltung in moderner Erzählkunst avantgardistischer und irgendwie surrealistischer Observanz. Weil diese ohne den Umweg über die Nachahmung vertrauter empirischer Wirklichkeit das Wesen

1 A.a.0., S. 63, zit. nach M.McLuhan: The Gutenberg galaxy, Toronto 1962 , S. 71 f.

2 H. Meyer: Raumgestaltung und Raumsymbolik in der Erzählkunst, in: Stud. Gen. 10 (1957), S. 620-630. 
zur Darstellung bringen will, tritt eine Verfremdung der Wirklichkeitselemente ein, die eine starke und eventuell bis zum Nullpunkt gehende Reduzierung von lokalen Angaben mit sich bringt, deren Sinn ja in ihrer potentiellen Wiedererkennbarkeit besteht. Diese Reduktion kann sich in zweierlei Richtung auswirken. Entweder wird mit dem Lokal auch die Raumsuggestion als solche abgeschwächt, der Schauplatz wird flächenhaft, die Gestalten agieren auf einer leeren Bühne wie die Puppen vor der flächenhaften Bühne eines Kasperletheaters (so im "Mann ohne Eigenschaften") ... Oder aber es treten Raumgestaltungen auf, die zwar in ihren einzelnen Elementen, nicht aber in ihrer jeweiligen Totalität an empirisch Gegebenes erinnern und gerade durch diesen ihren irrealen Charakter ihre Autonomie als Träger symbolischen Gehalts bekunden (so in Kafkas "Bau")." 1

H. Meyer charakterisiert die Phänomene der Häufung und Wiederholung bei Raabe. 2 "Bei Raabe sind die Möglichkeiten der Häufung differenzierter, indem diese entweder durch einfache Wiederholung oder durch Variation zustande kommt. Die bloBe Wiederholung kann zunächst einmal realpsychologisch im Affekt des Sprechenden begründet sein ... Interessanter noch ist die Wiederholungsfigur, wo sie nicht realpsychologisch, sondern formal erzählerisch bedingt ist und als kompositorisches Formprinzip dazu dient, die Sinnschwere eines Wortes zum Ausdruck zu bringen. Einige Hauptleitmotive ... bilden ein dichtes, sich durch den ganzen Roman hindurchziehendes Geflecht, das sich aber an bestimmten Zentralstellen, wo sie ausdrücklich zur Sprache kommen, knotenartig verdickt ... Die variierende Häufung kann rein verbaler Art sein, ohne daß die Aussage durch sie inhaltlich bereichert wird ... Durch die synonymische Häufung wird die Aussage keineswegs genauer, sondern vielmehr verschwommener ... Jean Paul spricht vom "farbigen Rand und Diffusionsraum fremder Beizüge", der bei Sterne durch die humoristische Paraphrase entstehe." 3

1 A.8.0., S. 622 .

2 H. Meyer: Raum und Zeit in Wilhelm Raabes Erzählkunst, 1953, zit. nach: Zur Poetik des Romans, Darmstadt 1965, S. 239-279.

3 A.a.0., S. $263 \mathrm{ff}$. 
Man kann also, und dies kann für ein Werk durchaus eine relevante Methode sein, nach der Angemessenheit von Zeichen und Raum fragen, wie man nach dem Verhältnis von Wortmaterial und jedem beliebigen Gegenstand fragt. Will man aber den Raum merkmalhaft aus jeder sonstigen thematisch zusammengehörigen Menge von Gegenständen hervorheben (und jeder Autor kann über den Raum sprechen wie über jedes andere Thema, ohne seine Raumkonzeption damit für das Werk strukturrelevant werden zu lassen), müssen Einheiten gefunden werden, die wesensmäBig rein zeitlicher Natur sind. Da in diesem Falle kein Zeichen von sich aus den Vorrang eines einschnittbildenden Elementes besitzt, wenn man nicht eine auBersprachlich vorgefabte Zeitkonzeption substituieren will, und da auf der anderen Seite ein mit einem Erzählvorgang verbundenes Zeichen, das der Analyse unterzogen wird, schon nicht mehr die Funktion offenbart, die es im Erzählkontext besitzt, ist die einzig gerechtfertigte Methode die, das Zeichen selber als eine zeitlich ausgedehnte Einheit zu betrachten. Ich tue dies auf der Grundlage des von $\mathrm{K}$. Müller formulierten Begriffs der Zeitstruktur. Er unterscheidet hier homogene und heterogene Sukzessionen. "Sind die Glieder einer Sukzession alle anschaulich gleich, dann können wir von homogenen Sukzessionen sprechen; im anderen Fall wären sie als heterogen zu bezeichnen. Diese Art von Homobzw. Heterogenität bestimmt sich - gemäB unserer Definition allein aus den Eigenschaften der Sukzessivglieder. Davon ist eine andere Definition von Homo- bzw. Heterogenität zu unterscheiden, die sich auf die Zeitstruktur der Sukzession bezieht. Wir können nämlich auch dann von homogenen Folgen sprechen, wenn die "Gliedzeiten" (d.h. die Dauer der Sukzessivglieder) sowie die Pausen der Zwischenzeiten sämtlich gleich sind. (Eine rhythmische Folge wäre in diesem Sinne eine heterogene Sukzession.) ... Die Sukzessivglieder heterogener Folgen können den verschiedenster psychischen Bereichen entstammen und dennoch als Teile eines einzigen Sinnzusammenhangs fungieren." 1

Betrachtet man Homogenität als das Merkmal, das alle Elemente eines Erzählwerkes zu Teilen eines übergeordneten Ganzen macht,

$1 \mathrm{A.a.0.,S.} 15$ 
so läßt sich Müllers Differenzierung der außerzeitlichen und der zeitbedingten Homogenität im literarischen Bereich fruchtbar einsetzen. Ein Beispiel der ersten Art von Homogenität ist Żeromskis "Vorfrühling", wo die Raummotive thematisch homogen sind, ihre Position zueinander jedoch statisch ist. Das zeigt ibre symmetrische Stellung an Anfang, Mitte und Schluß. Jede Handlungsphase ist repräsentativ für eine Entwicklungsstufe allgemeingültiger und geschichtlich relevanter Art. Auf der anderen Seite steht der Roman "Noce i dnie" von Maria Dębrowska, in welchem keine Handlungsphase mit einer Komponente der Welt, der historischen Epoche verbunden ist, sondern jede Erzählphase als zeitliche Einheit den Leser mit dieser Welt vertrauter macht. Zu dieser Gegenüberstellung von Zeichen als zeitlich ausgedehnten Einheiten und von Erzählphasen muB noch ein weiterer Gedanke hinzugefügt werden: genau differenzierbare zeitliche Abschnitte müssen nicht in jedem Pall einem bewußten Gestaltungsakt entspringen. Denn setzt man die Zeit des Lesers als primäre Zeitlinie an, so zeigt sich, daß diese in einer linięnförmigen, unstrukturierten Gestalt nicht vorliegt. Sie erhält bereits im voraus eine obligatorische Phasenstruktur, die sich einerseits auf die Phasenstruktur des Ablaufs psychischer Vorgänge stützt, andererseits aus einer in der Erzähltradition festgelegten und somit vom Leser erwarteten Abwechslung thematischer, bewegungsoder intensitätsbedingter Art ergibt. Diese Alternation wird sich zumindest als integrationstechnisches Hilfsmittel herausstellen. Bei der zeitlichen Organisation eines Erzählwerkes wird vom Autor sicher auch berücksichtigt, daß solche Eigenheiten des Erlebnisablaufes wie Spannung, Interesse, Konzentration usw. zeitlich begrenzt sind und nicht über eine gewisse Zeitspanne vom Leser erwartet werden dürfen. Daher darf man bei einer Erscheinung wie der Einfügung raumbezogener Erzählabschnitte in den dichten Verlauf der dramatischen Vorgänge die Frage nach dem Warum und dem strukturellen Effekt der Unterbrechung nicht überbetonen.

Diese Feststellung gibt den Grund dafür an, warum ein kontinuierlicher Wechsel von handlungs- und raumbezogenen Erzählphasen, 
wie sie in "Quo vadis" und "Chłopi" zu finden ist, überhaupt möglich ist, ohne als Bruch oder Diskontinuität empfunden zu werden. In "Quo vadis" zerreißen die raumbezogenen Erzählphasen oft den dramatischen Handlungsablauf, ohne die Spannung des Lesers zunichte zu machen (s.u.). 
4. Das Feld als Grundlage der Raumdarstellung

Die Feldtheorie betrachtet einen komplexen Realitätsbereich unter Bedingungen, unter denen eine einzelne Komponente normalerweise nicht, auftaucht. Entweder werden die Komponenten unter einem bestimmten Gesichtspunkt vereinheitlicht oder zu einem Zeitpunkt betrachtet, in dem sie - in der Kürze des Augenblicks - sonst nicht zusammen, zu einem Ganzen vereinigt, auftauchen.

Die Einführung der Feldtheorie oder genauer gesagt, verschiedener wissenschaftlicher Feldkonzeptionen, dient der literarischen Analyse zu folgenden Zwecken: 1. der Präzisierung der Wirksamkeit zeitlicher Kategorion am Objekt des Erzählens, indem nicht nur Rekurrenzstrecken zwischen den Zeichen und die Länge der Erzählphasen als relevante Größen anzusetzen sind, sondern auch nichtimmanente Phasen in Form von verschiedenen Darstellungsmöglichkeiten, von denen der Erzähler eine auswählt; 2. der Herausstellung von Realitätsbereichen, die auf den Erzählverlauf steuernde Wirkung ausüben, ohne expressis verbis in der Erzählung zu erscheinen. Es werden also Gegebenheiten wirksam, die nicht bezeichnet werden bzw. die als Ganzes in Funktion treten, obwohl sie nur als Spuren, in nichtadäquater Form, von Zeichen vertreten sind und so erscheinen.

Beide Punkte bedeuten eine Modifizierung und Kritik des Strukturbegriffe, indem eine rein immanente Betrachtungsweise, die sich nur an nennbare und ad hoc nachweisbare Elemente hält, zwischen denen dann meBbare und somit garantiert objektive Zeitverhältnisse bestehen, wesentliche Bereiche übergeht.

Dazu eine weitere methodische Bemerkung: Von den verschiedenen Feldkonzeptionen soll nicht die adäquateste, dem Erzählen nächstliegende ausgewählt oder gar eine spezifisch literarische Feldart konzipiert werden. Ist es aber sowohl von der Psychologie als auch von der Sprachwissenschaft erwiesen, daß sich bestimmte Gegenstände zu einem einheitlich wirksamen Ganzen zusammenschlieBen, bestimmte Gegenstände sich für diese feldmäßige Wirkung besonders gut eignen, dann besteht ganz sicher auch die Möglichkeit, 
den Raum beim Erzählen als Feld wirken zu lassen. Er ist dabei ein Ganzes, auch wenn er nur teilweise oder bruchstückhaft beschrieben bzw. genannt wird. DaB aber manche Gegenstände sich leichter in dieser Form zusammenschlieBen als andere, nicht wesensmäßig, sondern usuell oder traditionell, ist die Grundlage für die Tatsache, daß bestimmte Räume sich dem Erzähler von vornherein anbieten. Sein Eingehen auf den Raum ist nicht in jedem Fall als originelle Schöpfung zu betrachten, sondern auf der Grundlage traditioneller Raumtypen oder -topoi. Der letztere Begriff eröffnet einen weiteren Aspekt. Wenn es dem Erzähler möglich ist, neben Wörtern und Wortverbindungen ganze Darstellungsschemata zu übernehmen, so ist es auch möglich, das von ihm Neugeschaffene als Ganzes zu erleben und somit als Feld wirksam werden zu lassen. Einem solchen Wortkontext ist der Rang eines selbständigen Realitätsbereiches zuzumessen. Ein Raum erhält seine spezifischen Eigenheiten dann nlcht mehr im außersprachlichen, mit raumbezogenen Termini erfaßbaren Bereich, sondern er erhält Merkmale spezifisch sprachimmanenter Art.

Wenn ich die folgenden Kapitel mit Raumdarstellung, Raumfiktion und Raumgenese bezeichne, so sollen damit nicht verschiedene Erzähltjpen voneinander abgegrenzt werden. Vielmehr setze ich nacheinander diese drei Konzeptionen bei einem Erzählwerk an und untersuche dann, welche Schicht des Werkes jeweils in Erscheinung tritt, und ob es eine dieser Schichten ist, die als strukturrelevantes Aufbauprinzip mit einer bestimmbaren Berechtigung angenommen werden kann.

Um den Funktionsbereich des Feldbegriffs für sprachliche Phänomene abzustecken, stelle ich die verschiedenen Konzeptionen einander gegenüber. Dabei zeichnen sich zwei Hauptrichtungen ab. Die erste sieht das Feld als semantischen Bereich; ihre Vertreter werden zusammenfassend charakterisiert von S. Ohmann 1. Der Grundgedanke, wie inh besonders Jost Trier 2 formuliert, ist der, daB es zwischen dem einzelnen Wort und dem gesamten Wortschatz einer

1 S. Ohmann: Theories of the linguistic field, in: Word 9 (1953), S. 121-134.

2 J. Trier: Das sprachliche Feld, in: Neue Jahrbücher für Wissenschaft und Jugendbildung 10 (1934), S. 428-449. 
Sprache Zwischenglieder gibt, welche das Wortmaterial in bedeutungsmäßig zusammengehörige Einheiten aufteilen. Diese Einheiten sind Felder. "Es ist dann so, daB das Wort nur ist, weil es als Glied im Ganzen des Wortschatzes sein Wesen hat, des Wortschatzes, den wir uns auch nicht als einen Schatz, Vorrat, Thesaurus denken wollen, sondern - in einer architektonischen Analogie - als gebauten und gegliederten Raum, als Gefüge, das eben in seiner Gefügtheit und Gegliedertheit die Bedeutsamkeit jeder Bau- und Raumstelle (= des Wortes) setzt und bestimmt. Wir sagen: das Wort ergliedert sich aus dem Ganzen des gebauten, gegliederten Wortschatzes, und umgekehrt gliedert sich der Wortschatz aus in einzelne Worte ...... Felder sind die, zwischen Einzelworten und dem Wortschatz ganzen lebendigen sprachlichen Wirklichkeiten, die als Teilganze mit dem Wort das Merkmal gemeinsam haben, daß sie sich ausgliedern." 1

Eine Gliederung des Wortschatzes ist in dieser Form für eine bestimmte Sprache wohl möglich, wenn auch die Funktion des Wortes in konkreten Sprechakt sich nicht auf die eines Bedeutungsträgers beschränkt. Doch muß einer Gruppe von Wörtern, deren Zusammenhalt rein auf bedeutungsmäBigen Gemeinsamkeiten beruht, der Feldcharakter abgesprochen werden, solange sie als Glied betrachtet wird. Wäre sie ein Feld, so müßte die Veränderung eines Wortes das Entstehen eines neuen Feldes, also einer neuen Gruppe hervorrufen, während die Kontinuität des Bedeutungsbereiches gerade als Merkmal der Wortgruppe verwendet wird. Daß die außersprachliche Realität der wahrnehmenden und erlebenden Psyche in der Form von Feldern erscheint, hat eine Gliederung der Wörter zur Folge, die die Realität bezeichnen sollen. Ein solches Glied kann man deshalb aber noch nicht als Feld bezeichnen. Ferner tritt der Wortschatz als Ganzes bei Trier nie in Erscheinung, sondern nur augenscheinlich Zusammengehöriges wie Hond und Greifen. "Während Hand in greifen eindeutig gegeben ist, ist greifen in Hand nicht eindeutig gegeben: die Hand kann schlagen, sich ballen, drohen, winken, klopfen, streicheln, zeigen, schreiben, kurz, sie kann in eine ganze Reihe anderer Bedeutungsbeziehungen eingehen. Sie ist

1 A.a.0., s. 430 f. 
eben keine Situation, sondern sie ist Wesen, und als solches möglicher Teilhaber an sehr verschiedenen Situationen." 1

Nun gehört aber unbestreitbar auch eine Situation vom Typ "ich greife", also der Satz, zum Wesen der Sprache. Diesen Sachverhalt versucht W. Porzig durch die Gegenüberstellung von parataktischen und syntaktischen Feldern zu erfassen 2. "Die völlig eindeutigen Zuordnungen wie die von Zahn und beiBen sind nur äuBerste Fälle, an denen uns die Beziehung bewuBt wird. Aber in der Tat haben alle Wörter gewissermaßen ein Kraftfeld um sich, in das Wörter nur ganz bestimmter Art eindringen können. Jedes Verbum kann nur einen ganz bestimmten Kreis von Subjekten und, wenn es transitiv ist, von Objekten haben; auch die sonstigen Bestimmungen, mit denen es im Satze zusammentrifft, werden durch seinen Inhalt bestimmt. Umgekehrt kann man von jeder Person oder Sache nicht alles mögliche, sondern nur gewisse Dinge aussagen, nur gewisse Handlungen kann man mit oder an ihnen vornehmen. $\mathrm{Zu}$ jedem Gegenstand gibt es eine Gruppe von Eigenschaftswörtern, die zu ihm passen, und jedes Eigenschaftswort paßt nur zu best1mmten Gegenständen. Der Kreis dieser Möglichkeiten kann kleiner oder größer sein, von der eindeutigen Zuordnung zur scheinbar unbegrenzten Allgemeinheit, aber grundsätzlich kann man ihn immer angeben, und die großen einsprachigen Wörterbücher tun das auch, weil eben nur so der Inhalt eines Wortes genau bestimmt werden kann. So stellt der Bereich der Wörter, die mit einem gegebenen Wort sinnvoll verknüpft werden können, auch ein Wortfeld dar. Zum Unterschied von den vorhin geschilderten parataktischen kann man Felder dieser Art syntaktische nennen." 3

Der Satz, daB man von einem Gegenstand oder einer Person nur gewisse Dinge aussagen kann, daß nur eine beschränkte Zahl von Eigenschaften zu ihnen paßt, mit anderen Worten: daB sie sich jeweils aus einer beschränkten Zahl von Eigenschaften zusammensetzen, gilt auch für den auBersprachlichen Bereich. So tritt auch hier

1 A.a.0., S. 442 .

2 W. Porzig: Das Wunder der Sprache, Bern 19572, besonders Kap. 4: Die Gliederung des Wortschatzes, S. $117 \mathrm{f}$.

3 A. a.0., S. $124 \mathrm{f}$. 
das Ergebnis ein, daß mit zunehmender Präzision der Differenzierung von Feldem im Sinne von Gliedern eines Bedeutungsbereiches zwar das sprachlich lexikalische Zuordnungssystem präzisiert wird, die Darstellungsfunktionen und somit der mediale Charakter der Sprache außer acht gelassen wird. Auch diese Feldkonzeption, die grammatikalische Kategorien als Ordnungsfaktoren berücksichtigt, kann wohl das Wortmaterial für die Bezeichnung räumlicherGegebenheiten für eine Sprache differenzieren, ohne jedoch dem Funktionieren dieser Wörter über ihren Zeichencharakter hinaus im aktuellen Erzählzusammenhang die Wege aufzuzeigen.

Als Ergebnis für das Problem der Raumdarstellung kann man aus dieser Konzeption der Wortverbindungen die Tatsache verwenden, daB für eine räumliche Gegebenheit, sobald für diese ein Wort parat ist, auch eine Anzahl naheliegender, verwandter oder spezifizierender anderer Wörter mitgegeben ist, die aber, und darauf kommt es an, nicht notwendigerweise den spezifisch räumlichen Charakter dieser Gegebenheit betreffen.

Dies ist in Rejmonts "Chłopi" nicht nur in einigen Raumdarstellungen der Fall, es ist sein Erzählprinzip schlechthin. Er beschreibt eine räumliche Naturgegebenheit, z.B. die Landschaft, den Wald, das Dorf usw., entfaltet dabel aber eine den Leser überraschende und beeindruckende Vielfalt von Eigenschaften, die mit der spezifisch räumlichen Aufteilung der Natur nichts zu tun haben. Diese Wörter, die auf dem Wege des literarischen Vergleichs oder einer sonstigen Figur mit in das Bild aufgenommen werden und dieses erst entstehen lassen, bauen somit den spezifischen Raum in seiner ganzen Vielfalt auf, selbst wenn manche Einzelheit, wie die genaue Analyse zeigt, sich räumlich nicht einflugt bzw. vom räumlich perspektivischen Standpunkt aus gesehen nicht möglich ist. Legt man diesem Werk eine rein darstellende, objektorientierte Konzeption zugrunde, so zeigt sich ein Autor, der der Fülle des Gegebenen und Darzustellenden kaum gewachsen ist. Diese Methode wendet der Autor nicht bewuBt als Technik an. Vielmehr kann die Analyse, die eine reine Darstellungsfunktion zugrunde legt, auf diese Weise das Phänomen der Naturverherrlichung und -idealisierung genauer beleuchten. 
Auf der anderen Seite ist auch mit dem Wort für einen Gegenstand, für welchen die räumliche Umgebung irrelevant ist, ein Feld von bedeutungsverwandten Wörtern gegeben, von denen das eine oder andere der Bezeichnung räumlicher Gegebenheiten dienen mag. Die Beantwortung der Frage, mit welcher Relevanz der Autor den Raum als abstrakte Kategorie einsetzt, ist damit nicht mehr nur Selbstzweck. Sie kann vielmehr die Motivation beleuchten, die zur Einfügung von Raumdarstellungen führt, ohne daB dieser Raum für die eigentliche Romanhandlung vonnöten ist. Der Raum kann dabei als Vehikel fungieren, das der Einführung eines Wortmaterials mit stilistischen und künstlerischen Effekten ohne Relevanz der räumlichen Kategorie an sich dient. Żeromskis "Vorfrühlingsszenen" dienen nicht der Einführung verschiedener Schauplätze, sondern ihre stilistisch und rhetorisch überreiche Ausführung isoliert sie geradezu vom übrigen Handlungsverlauf; aber sie verleihen diesem durch ihre Position an Anfang, Mitte und Schluß eine symmetrische Gestalt.

Porzig erläutert den Feldcharakter solcher Gruppen an den Beispielen der Felder: Farbe, Himmelskörper, Jahreszeiten, Witterung, Verwandtschaftsbezeichnungen u.a. Für die räumliche Problematik ist besonders das Wortfeld "Landschaft" interessant. "Namentlich wenn die Sachen ebenso wie ihre Bezeichnungen von Menschenhand geschaffen sind, also bei allen Geräten, entspricht die Gliederung der sprachlichen Felder genau der technischen Gliederung der Geräte, etwa bei den Teilen des Wagens oder den Werkzeugen in einer Werkstatt. Wenn wir dagegen das Wortfeld "Landschaft" betrachten, so entsprechen die einzelnen Glieder gewiß den Zügen der Wirklichkeit, es gibt Berge, Täler, Hänge, Mulden, Schluchten, Sättel, Kämme, Gipfel und vieles andere; aber daß sie aus der Landschaft herausgehoben und gerade so und nicht anders herausgehoben werden, das ist Menschenwerk, das die Natur in menschliche Umwelt verwandelt. Wenn man versucht, den Inhalt dieser Wörter zu bestimmen, merkt man, daB man alle andern braucht, ein sicheres Zeichen für die Zusanmengehörigkeit des Feldes." 1

1 A.a.0., S. 118 . 
Für die Gegenüberstellung einer Gliederung des Wortschatzes und einer Gliederung der Wirklichkeit bedarf es nicht des Feldes; daß aber aus der Landschaft etwas "herausgehoben" wird, was an und für sich ein konstituierendes Moment der Landschaft ist, betrifft den spezifischen Charakter des sprachlichen Gegebenseins. Und daß mit einem der Elemente alle übrigen mitgegeben sind, wobei sich durch die Veränderung des einen alle übrigen mitverändern, ist das Wesen des Feldes. Denn dieses ist nur als Ganzes wirksam, und die Gruppen, die nur gemeinsam in Erscheinung treten, fallen unter die Begriffsdefinition des Feldes.

Die spezifisch sprachliche Eigenart der feldmäBigen Verbindung ist nicht auf die Zeichen als Bedeutungsträger beschränkt. Diese Erkenntnis liegt auch den oben behandelten Feldkonzeptionen zugrunde, wenn auch Bedeutungs- und Vertretungsfunktionen des sprachlichen Systems mehr oder weniger ausschlieBlich im Vordergrund stehen. Das ist auch der Fall in der Theorie Gert Müllers 1, wenn er durch die Unterscheidung von Wortfeld und Sprachfeld das Auftreten eines Wortes im Satzzusammenhang berücksichtigt. Die gleichberechtigte Berücksichtigung beider Sprachfunktionen, der Bezeichnung und der Darstellung eines Dinges oder Sachverhaltes, ist das Verdienst von $K$. Bühler; sie findet ihren Niederschlag in der Zweifelderlehre seiner Sprachtheorie.

"Die Nennwörter fungieren als Symbole und erfahren ihre spezifische Bedeutungserfüllung und -präzision im synsemantischen Umfeld; ich schlage den Namen Symbolfeld für diese andere, keineswegs mit den Situationsmomenten zu verwechselnde Ordnung vor." 2 Und aus den Situationsmomenten setzt sich das Zeigfeld zusammen. "Beim Zeigen können sprechende Menschen nichts anderes als ausnützen, was ihnen das Zeigfeld bietet, versteht sich mehr oder weniger davon, aber nichts, was derjenige, der das Zeigfeld kennt, nicht vorauszusagen oder, wo es da ist, einzuordnen vermöchte ... Es ist also mit den Sprachzeichen so, daß sie im "Alltagsverkehr" in das Feld der Sprechsituation eingesetzt bestimmte Feldwerte

1 G. Müller: Wortfeld und Sprachfeld, in: Festschrift für Ernst Otto, Berlin 1956, S. 155-163.

2 A.a.0., S. 81. 
erhalten." 1

Als Unterschied zu den vorangegangenen Feldtheorien kann man festhalten: die Elemente, aus denen sich diese Zeigfelder zusammensetzen, sind weder ausschlieBlich im Bereich des Zeichens noch im Bereich des Bezeichneten zu suchen. Die synsemantische Umgebung eines Wortes kann zwar mit Wörtern bezeichnet, die Feldstellen also mit lexikalischen Einheiten besetzt werden, doch kann seine Wirkung als Ganzes im Sprechakt auch ohne diese Differenzierung nachgewiesen werden. Ein gleiches gilt vom Zeigfeld: die einzelnen Komponenten der Situation können in Form von Dingen und Sachverhalten spezifiziert werden, doch sind sie in der konkreten Sprachsituation nur als Ganzes für die Bedeutungserfüllung des Wortes bzw. der Wortfolge relevant.

Natürlich finden wir diese deiktischen Felder auch im Erzählwerk, wenn z.B. ein Dialog nur verständlich ist auf dem Hintergrund der räumlichen, szenischen, schauplatzeigenen Situationsumgebung oder der Situation, die sich aus dem Vorangegangenen ergibt. Aber wir finden auch die ungekehrte Erscheinung, daß das Feld nicht als Hilfsgröße, als Orientierungshilfe dient, sondern im Verlauf des Werkes aufgebaut wird, also das Ziel des Erzählens bildet. Ein Beispiel für diese Technik als Erzählprinzip ist Dąbrowskas Roman "Noce i dnie". Die zeitliche Diskontinuität der Einzelszenen lenkt die Aufmerksamkeitskonzentration von den einzelnen Geschehnisabschnitten ab. Der Raum aber, der ibnen als Schauplatz dient, wird um eine Komponente reicher, dem Leser vertrauter und steht am Ende als eine lebendige Größe vor dem Auge des Lesers, ohne daß er als Ganzes jemals von der Autorin genannt worden wäre.

Zweifellos liegt auch beim Erzählen eine Sprechsituation vor. Dabei muß grundsätzlich unterschieden werden zwischen der Situation, die sich aus dem Gegenüber von Sprecher und Zuhörer bzw. im Falle des Erzählwerks aus dem korrespondierenden point of view von Autor, Leser und Figur ergibt, und der Situation, die zu einem bestimmten Zeitpunkt im Erzählvorgang aus der Natur des erzählten Vorgangs und der damit verbundenen Zeichen resultiert. Für diese wird die ausschlaggebende Komponente von den "artikulierten

1 A.a.0., S. 83 f. 
Tönen in der Zeit", welche Lessing als die Grundlage sprachlichen Darstellens postuliert hat, gebildet. Die erste aber bildet den deiktischen Bereich, in welchem das Ich, das Hier und das Jetzt anzutreffen ist. Die verschiedenen Möglichkeiten der Bedeutungserfüllung sind damit noch in keiner Weise berührt; für alle sind jedoch Weg und Grenze dadurch festgelegt, daß alle Symbole eines Feldes und jedes Feld der Symbole bedurfen.

Von diesen Feldarten muß der Begriff des Umfeldes geschieden werden; das Umfeld ist der Bereich der Wirksamkeit von Relationen, in denen sich der bezeichnete Gegenstand befindet. "Es gehört zu den nie ganz übersehenen oder geleugneten Tatsachen, daß die Sinnesdaten nicht isoliert, sondern eingebettet oder eingebaut in wechselnde "Ganzheiten" des psychologischen Geschehens aufzutreten pflegen und von dorther wechselnde Modifikationen erfahren. Dafür bot sich der Name "Unfeld" wie von selbst an und hat sich eingebürgert ... Das Einzelne erscheint mit anderen Sinnesgleichen im Verbande und der Verband erweist sich als wirksames Umfeld." 1

In diesem Bereich, den um einen gegebenen Gegenstand sich gruppierenden verwandten Gegenständen sowie den mit einer bestimmten Vorstellung sich verbindenden naheliegenden anderen Vorstellungen, können räumliche Ordnungskomponenten wirksam werden. Daß diese räumliche Anordnung durchaus nicht gleich ist mit der Position im Raum oder dem Raumteil, welchen der betreffende Gegenstand innehat, sondern eine Konstruktion für einen ganz bestimmten Zweck sein kann, hat $\mathrm{K}$. Duncker am Modellfall des Suchens untersucht. W. Metzger 2 berichtet darüber: "In der Regel ist das Suchen (auch ein inneres) auf etwas gerichtet, was seiner Art nach ein mehr oder weniger ausgedehnter Teil eines "Feldes" oder irgendwie sonst geschaffenen "konkreten Bereiches" ist bzw. sein kann, der von den Suchenden durchlaufen wird. Der Suchbereich wird oft als die eigentliche "Sphäre", als natürliches Umfeld eines bestimmten Gegenstandes mit diesem zusammen wachgerufen; er kann aber schon

1 R. Bühler, a.a.0., s. $154 \mathrm{f}$.

2 W. Metzger: Das Raumproblem in der Psychologie, in: Stud. generale 10 (1957), S. 542-552. 
als Bestimmungsstück in der Aufgabe enthalten sein. Neben den räumlichen Feldern (einer Landschaft, einem Buch) kommen zeitliche Felder (ein Tag, ein Lebensabschnitt, eine Unterrichtsstunde) vor." 1

Die Möglichkeiten der Psyche, auch unräumliche Gegebenheiten räumlich anzuordnen bzw. räumliche Gegebenheiten in unräumliche Felder zu gruppieren, bezeichnet Duncker als topische Antizipation, bzw. die Verwandlung eines Strukturorts des Gegenstands in eine Eigenschaft. "Es genüge abschließend der Hinweis, daß der Ratende, Suchende, Nachdenkende sich im Rahmen von Aufgaben der angedeuteten Art regelmäBig in mehr oder weniger weite und bestimmte Gebiete hineinbegibt und in ihnen umherwandert; diese Gebiete findet er, - wenn wir die Sache einer sorgfältigen phänomenologischen Analyse unterziehen - durchaus nicht in sich selber vor, sondern er tritt in sie ein, er bewegt sich in ihnen und läßt sie wieder hinter sich." 2

Die Verwendung dieser Art von räumlichen Feldern als relevantes Aufbauprinzip eines Erzählwerkes wird im folgenden Kapitel, das die Raumfiktion zum Gegenstand hat, beschrieben. Ich habe den psychologischen Begriff des Umfeldes der Vollständigkeit halber hier vorweggenommen.

Die Gegenüberstellung dieser beiden Feldarten, der Felder des sprachlichen Darstellungsgerätes und der Unfelder der bezeichneten Dinge und Sachverhalte, ergibt folgendes für die Darstellung des Raumes: mit dem Zeichen ist zugleich ein Bereich abgesteckt, innerhalb dessen sich seine Bedeutung nur realisieren kann bzw. präzisieren läBt. Im Erzählwerk erscheint das Zeichen als Glied einer Sukzession und der Bedeutungsbereich als ein Feld gemäB der Definition von K. Lewin: "Eine Gesamtheit gleichzeitig bestehender Tatsachen, die als gegenseitig voneinander abhängig begriffen werden, nennt man ein Feld." 3 tberdies ist das Zeichen ein-

1 A.a.0., S. 543 .

2 A.a.0., S. 544 .

3 K. Iewin: Feldtheorie in den Sozialwissenschaften, Bern 1963, S. 273. 
gebettet in eine Anzahl anderer Zeichen, der vorausgehenden und der folgenden, welche als kontexbedingtes Umfeld zusammenwirken und den Gliedcharakter des Zeichens bestimmen. Diese beiden Komponenten hat E. Lämmert mit "Stellenwert im Ablauf" und "Vorgangsbildung" als die sich überschneidenden Funktionen bezeichnet. "Indessen ließe sich gerade von der Beobachtung der Rück- und Vorverweise aus eine systematische ErschlieBung der Zusammenhänge in die Wege leiten, die zwischen einzelnen Symbolen, Leit- und Begleitmotiven, ornamentalen und allegorischen Bildern und dem eigentlichen Erzählvorgang bestehen. Solche Erzählglieder besitzen eine spezifisch künstlerische Eigenmächtigkeit und charakterisieren die Erzählweise in hohem Maße. Sie haben ihren - oft durch Wiederholung noch markierten - Stellenwert im Ablauf; darüber hinaus ist aber ihre energetische Wirkung auf die Vorgangsbildung und ist vor allem ihre sinnverbindende und vertiefende Tendenz im Augenblick ihrer Darbietung von entscheidender Bedeutung." 1

Ein Erzählwerk wäre aufgmund dieser Bemerkung ein ungeheuer vielfältiges Gefüge von Beziehungen und Verbindungslinien, die überdies noch in Form eines hierarchischen Systems angeordnet werden müBten. Wendet man noch dazu die Feldtheorie auf den Raum an, so scheint eine zusätzliche Zahl von Verbindungen zu außerhalb liegenden und trotzdem werkimmanenten Elementen hinzuzukommen. Doch zeigt sich gerade dadurch eine Vereinfachung der Verhältnisse. Die Feldkonzeption zeigt den Autor konfrontiert mit einem Objekt, einem räumlichen Komplex. Er versucht diesen sprachlich zu erfassen, aber nicht das Ergebnis oder das Gelingen dieses Versuchs ist strukturrelevant, sondern der Versuch an sich kann zum Gestaltungsprinzip werden. Die Raumdarstellungen in Prus' "Lalka" zeigen ein betont undeutliches und unklares Ergebnis, doch der Raum als selbständige Kategorie verhilft der Figur, in deren Perspektive der Versuch verlegt ist, zu ihrem eigenen und charakteristischen Profil. Der Held versucht, Paris darzustellen. Das Ergebnis ist absolut unzureichend, aber seine seelische Situation wird aufs genaueste analysiert. In Iwaszkiewicz' Novelle "Die

1 E. Lämmert: Bauformen des Erzählens, Stuttgart 1955, S. 244. 
Mühle an der Utrata" ist es der Versuch, ein ganz eigenartiges Raumbild zu erfassen, der jeweils kurz vor dem Gelingen abbrechen muß. Der Autor bzw. die Figuren erleben zwar den Raum, doch weicht das sprachliche Formulierungsergebnis stets vom tatsächlich Erlebten $a b$, so daB es nie zu einer vollen Ubereinstimmung kommt. Erst der Schluß des Werkes bringt das Gelingen dieses Versuches, und in zweiter Linie ist dieser Moment des Gelingens der Todesmoment des Helden.

In Schulz' Werk werden auf dieser suchenden Darstellungsebene eine Reihe von Termini eingeführt. Diese erfassen mit ihrer begrifflichen Seite auch den Raum, wirken dann allerdings in ihrer spezifischen Anordnung auf ganz andere Art, die ich mit dem im folgenden Kapitel einzuführenden Begriff der Raumfiktion charakterisiere. Diese Fiktion ist aber erst auf der Grundlage der ursprünglich darstellenden Autorenfunktion möglich.

In Andrzejewskis "Bramy raju" deckt die Suche nach der objektorientierten Raumdarstellung eine Schicht von rhetorischen und literatischen Figuren auf. Die Konzentration dieses Materials weist ebenfalls über sich hinaus. Die medial entstandenen Ebenen und Sphären metaphorischer und symbolischer Art werden auf dem Weg der Raumgenese (s. 6. Kapitel) zu konstituierenden Ordnungsmomenten einer konstruierten Welt, der künstlichen Welt des ideologisch manipulierten Wunschdenkens.

Das Raumdetail ist aber nicht unbedingt durch das zugrundeliegende Auswahlprinzip charakteristisch für den Autor, indem es ihn aufgrund seiner Funktion identifiziert. Es kann auch in seiner betonten Detailhaftigkeit die Illusion des ganzen Raumes wachrufen. W. Killy beschreibt die Funktion des Details in Goethes "Wahlverwandtschaften" folgendermaßen: "Die Einzelheit an sich ist ohne Interesse, sie realisiert sich sinnvoll erst im Ganzen, und nichts wäre Goethe gleichgültiger, ja sinnwidriger erschienen als die Abbildung von einmaligem Detail und Milieu als solchem, mit welcher man seit dem 19. Jhdt. die Wahrscheinlichkeit der poetischen Erfindung zu beglaubigen sucht. Wir erfahren nicht, wie die Landschaft in Wirklichkeit aussieht. Wenn aber eine allgemeine Anschauung differenziert und dem Leser bestimmte Züge 
berichtet werden, so darf er ihres bedeutenden Wesens sicher sein." 1

Die Wahrscheinlichkeit der poetischen Erfindung darf nicht als Charakteristikum einer bestimmten literarischen Epoche angesehen werden; vielmehr ist die Ausnützung der Illusionsfähigkeit eine Technik, die bei jeder sprachlichen Raumdarstellung die Grundlage bildet. Dabei muß graduell unterschieden werden, wieviel davon ausgenützt wird. Brinkmanns Charakteristik der "echten Sachlichkeit", ebenfalls auf die "Wahlverwandtschaften" bezogen, kann sich also höchstens auf eine weltanschauliche, nicht eine darstellungstechnische Komponente beziehen: "Immer also bestimmt echte Sachlichkeit im höchsten Sinne, reine Objektivität diesen Stil, niemals empirische Neugier, der das Einzelne um seiner selbst willen wichtig wäre, niemals die subjektiven Stimmungen, Bedürfnisse, Wünsche, Leidenschaften der dargestellten Personen." Der Autor kann also die räumlich relevanten Elemente auswählen und zur Darstellung bringen, er kann ebensogut betont Unwichtiges, für den Charakter des Raumes Irrelevantes in den Erzählverlauf einflechten: beide Techniken werden vom sprachlichen Aspekt aus betrachtet elliptischer Natur sein und keine kann den Vorrang künstlerischer Raumdarstellung für sich beanspruchen. "Es gibt auch eine Hochkultur des "elliptischen" Sprechens, wobei zur Erfüllung und Präzisierung des Sinnes der Lautinseln die Feldwerte der Situation ausgenützt werden." 3

1 W. Killy: Wirklichkeit und Kunstcharakter, München 1963, s. 22.

2 R. Brinkmann: Wirklichkeit und Illusion, Tübingen 1957, S. 297.

3 K. Bühler, a.a.0., s. 88 . 
5. Die Rolle des Raumes im EntstehungsprozeB der literarischen Fiktion

In diesem Kapitel erscheint das Erzählen unter einem anderen Aspekt: als einheitlicher Vorgang, relativ unabhängig vom Erzählgegenstand. Die Autorenfunktion, sich von einer bestimmten Perspektive aus heterogenen und beziehungslosen Objekten zuzuwenden und sich von ihnen wieder abzuwenden, tritt in den Hintergrund; das Nebeneinander dieser Objekte weicht der Relevanz des Hintereinander von Erzählphasen, diese haben ihren Stellenwert im Gesamtablauf. Unter diesem Gesichtspunkt der Verlaufsbedingtheit und der zeitlichen Eingrenzung durch das Vorangehende und das Nachfolgende wird das sprachliche Material nicht mehr als durch Felder ergänzt betrachtet, sondern es bildet selber einen vollständigen Bereich, der seinerseits für folgende Erzählphasen als Feld wirksam werden kann.

Es handelt sich - gegenüber dem im vorigen Kapitel angesetzten medialen Darstellen - insofern um einen neuen Aspekt, als eine sprachliche, semantisch oder thematisch bedingte Einheit nicht mehr für sich betrachtet wird und zu ihrer Bedeutungserfüllung der Felder bedarf, die einen Kreis von möglichen Realisationen abgrenzen oder nahelegen. Den Einheiten dieser Art stehen Einheiten als ausschlieBlich sprachliche Gegebenheiten mit einer zeitlichen Begrenzung gegenüber; sie sind durch die Selbständigkeit charakterisiert, aufgrund derer sie von den nachfolgenden Einheiten der gleichen Art und der gleichen zeitlichen Größenordnung ergänzt werden und mit diesen zusammen einen einheitlichen Vorgang bilden. Räumliche Elemente, die in dieser Form an den Leser herangetragen werden, verbinden sich zu einem Ergebnis, das ich mit Fiktion bezeichne.

Dazu sollen noch einmal die Erzählphasen in Sienkiewicz' "Quo vadis" herangezogen werden. Unter dem Aspekt des raumbezogenen Darstellens findet man Einheiten, die durch ein verwandtes Wortmaterial geprägt sind, entweder lateinische Lokalitätsbezeichnungen oder Nomina und Adjektive einer bestimmten Kategorie, die auf Größenordnung und Erlebnisdimensionen ausgerichtet ist. Der Aspekt der Fiktion wird nun der Tatsache gerecht, daß ein auf diese Weise dargestellter Raum nicht von seinem Platz im Erzählvorgang iso- 
liert betrachtet und nachgeprüft werden kann, wenn nicht der zeitliche und damit strukturelle Rahmen durchbrochen werden soll. Das Voranschreiten der Fiktion als kontinuierlicher Vorgang ist durch den Aufbau einer außergewöhnlichen, exotischen Welt charakterisiert, in und durch die der Leser geführt wird. Diesen Effekt erzielt der Autor, wie unten gezeigt wird, sowohl durch Beschränkung auf ungewöhnliche Räume, als auch durch ungewöhnliche Handlungen und Ereignisse, ohne daB das Nebeneinander dieser verschiedenen Gegebenheiten durch eine erzähltechnisch gesonderte Behandlung motiviert ist. Die Raumdarstellung ist topisch vorgeprägt und trägt in keiner Weise die Eigenschaften künstlerischer Neuschöpfung. Die Folge von spannungsgeladenen, überraschenden Erzählphasen ist die Leistung des Autors, und sie resultiert in der Fiktion eines für den Leser neuen und ungewöhnlichen Raumes.

Rom ist schon von sich aus für den zeitgenössischen Leserkreis eine exotische und ungewöhnliche Welt. Warschau, der Schauplatz des Romans "Lalka", wird als dem Leser bekannt vorausgesetzt und tritt unter dem Aspekt der Darstellung nicht oder nur in Spuren in Erscheinung. Die hier auftauchenden raumbezogenen Erzählabschnitte treten nur im Verband mit dem Erlebnis der Figuren, als ihre Träume und Visionen, in Erscheinung und haben nur in deren zeitlicher Umgebung ihren Sinn. Den fiktiven Charakter dieser Räume bringt der Autor auch ganz klar zum Ausdruck, sie sind stets verbunden mit den Wendungen: es war ihm, als ob..., er stellte sich vor, daß..., usw. Man kann also sagen, die auktorale Intention ist die Fiktion einer Figur oder des Seelenzustandes einer Figur, und der Raum leistet zu dieser Fiktion einen Beitrag. Nur in diesem Zusammenhang erfüllen die Räume ihre strukturrelevante Funktion.

Beim Erzählen muß also gerade der umgekehrte Vorgang angesetzt werden als beim Sprechen, und zwar nicht als Gegensatz, sondern als Ergänzung und Weiterführung. Das gesprochene Wort wird durch die Situation ergänzt. Diese Situation ist auch der Maßstab für den Zeitpunkt, an dem es zu lokalisieren ist. Beim Erzählen ist das Wort eine selbständige, durch nichts zu ergänzende Einheit, die im obligatorischen zeitlichen Verband mit ihresgleichen auf- 
taucht, durch diese ergänzt wird, sie aber in gleichen Maße ergänzt.

Dieser Fiktionsvorgang, der Elemente verschiedenartigster Herkunft, also die verschiedensten Zeichen, zusammenfügt und zu einer zeitlichen Einheit werden läßt, kann auch räumliche Elemente, raumbezogene Zeichen integrieren; sie werden zu Hilfsmitteln der sprachlichen Fiktion. Auf der anderen Seite ist auch die Fiktion des Raumes ein sollch zeitlich ausgedehnter und begrenzter Vorgang, an dem auch Elemente sprachlicher Art teilhaben können, die für sich betrachtet keinen Bezug zu räumlichen Gegebenheiten haben. Setzt man den Raum in dieser Form in die Reihe der Fiktionsmöglichkeiten ein, so muß man genau untersuchen, wann und unter welchen Bedingungen der Autor dem Raum als Erzählgegenstand so begegnet, daB ein Weitererzählen auch außerhalb der spezifisch räumlichen Ebene dem Raum zugutekommt. Sehr gut kann man das an Reymonts Naturbildern verfolgen, wo auf dem Wege des literarischen Vergleichs eine zusätzliche Reihe von Elementen den ursprünglichen Raum überlagern und die Ausprägung des Raumes fast ausschließlich übernehmen. Sind es hier noch die neuen Gegenstände, die mit den Mitteln der literarischen Figur miteinbezogen werden, aber der gleichen Sphäre, der Natur entstammen, so können es auch Gegenstände artfremder Umgebung und in letzter Konsequenz nur die Zeichen sein, die das Fiktionsergebnis bilden.

Die Analyse am Einzelwerk kann diese Umstände und Bedingungen finden, unter denen das Nebeneinander von raumbezogenen und räumlich irrelevanten Erzählphasen zu einem einheitlichen Fiktionsvorgang integriert wird. Die Theorie kann die psychischen Bedingungen für die Tatsache erhellen, daB auch spezifisch nichträumliche Elemente eine räumliche Anordnung erfahren können, daB der Raum a uch durch nichtraumbezogene Zeichen in einem einheitlichen zeitlichen Rahmen darstellbar ist, und daß eine Korrespondenz von Räumen und seelischen Zuständen in die Struktur des Erzählens Eingang gefunden hat und hier als in der Geschichte und Tradition der Literatur geformtes Prinzip seinen festen Platz einnimmt. 
6. Die werkimmanente Raumgenese als die spezifisch literarische Erscheinungsform des Raumes

Bei der bisherigen Betrachtung des Raumes im Erzählzusammenhang wurde die Frage nach künstlerischen Effekten und ästhetischen Werten berrabt hintangesetzt oder vermieden. Dadurch gelang es, eine isolierte Analyse raumbezogener Zeichen und Erzählphasen zu vermeiden, sie also nicht als an sich problematisches objekt anzusehen, sondern als Verständnis- und Orientierungshilfen, als Integrationsbestandteile oder als Realisationen in der Tradition vorgegebener Modelle. Die Frage der Motivation stand im Vordergrund: Wer stellt den Raum dar, an welchen Positionen befinden sich diesbezügliche Wörter und Abschnitte, welche Schicht des Erzählvorgangs erhält durch ihn Merkmalhaftigkeit und Abgrenzung. Im folgenden Kapitel steht die spezifisch künstlerische Seinsweise oder Funktion des Raumes zur Debatte. Damit verbunden ist die Frage nach der Seinsweise und Gesetzmäßigkeit des Erzählwerkes überhaupt und nach der Rolle des Raumes bei der Bestimmung der spezifischen Seinsweise des Erzählens. In diesem Zusammenhang stellen sich auch die Probleme, wie der Raum aus jeder anderen Menge von Gegebenheiten hervorragt, wodurch er sich von jedem anderen Komplex zusammengehöriger Elemente unterscheidet, also letzten Endes die Definitionsfrage. Die Lösung dieser Frage wird so aussehen, daß der Raum hier, verglichen mit den vorangegangenen Phänomenen der Raumdarstellung und der Raumfiktion, nicht komplizierter oder vielfältiger in Erscheinung tritt, sondern einfacher, kompakter, eben als die Kategorie, die zur notwendigen Bestimmung jedes Realitätsbereiches vonnöten ist und der an Allgemeinheit nur die Zeit zur Seite steht.

Die zeitliche Kategorie, im Erzählwerk angesetzt, zeitigt zunächst einen einheitlichen Vorgang von einer Größenordnung und Geschlossenheit, wie sie nur hier in dieser Form vorkommt. $\mathrm{Zu}$ messen ist diese Dimension, wie die Analyse des Fiktionsvorgangs ergeben hat, nicht chronometrisch, sonderm mit verwandten oder identischen Zeiteinheiten, deren Abstand voneinander durch die Anzahl der dazwischenliegenden Einheiten bestimmt ist. Diese Art der Messung hat $K$. Knauer bei der Untersuchung lautlicher 
Erscheinungen angewendet und mit Rekurrenz bezeichnet. 1 Er greift zum Vergleich mit der Perlenkette, wenn er die Sprache als lineares Zeitmuster charakterisiert. "... es tritt, wenigstens im Grund- und Normalfall, an einem gegebenen Zeitpunkt nur ein sprachliches Ereignis ein ... Es handelt sich vergleichsweise um Perlen eines Vorrats von begrenzt vielen verschiedenen Perlen, die an einem Faden in unser Blickfeld geschoben werden, und uns sowohl einzelne Eigenschaften (GröBe, Gestalt, Farbe) wie verschiedene Anordnung zeigen. Der Begriff der Anordnung umschließt den der Rekurrenzstrecke zwischen zwei je in zu bestimmendem Sinne gleichen Perlen; diese Strecke wird z.B. durch die Anzahl der dazwischenliegenden oder eine Auswahl der dazwischenliegenden Perlen gemessen. Die Antwort auf die Frage, ob in einem gegebenen Text Rekurrenzen, in welcher Ordnung Rekurrenzen auftauchen, ist die Antwort auf die Frage nach wichtigen Eigenschaften der Kette." 2 '

Der Aspekt, der das Erzählwerk als eine geschlossene zeitliche Größe betrachtet, hat auch seine Auswirkungen auf dem räumlichen Sektor. So wie ein zeitlicher Abschnitt von dieser Größenordnung nur in einem Erzählwerk vorkommt, ist auch der damit verbundene und in dieser Zeit sich entfaltende Effekt räumlicher Art für das Erzählen spezifisch. Das Entstehen des Raumes, hier zu verstehen als Kategorie im Euklidschen Sinne, ist an einen zeitlichen Vorgang gebunden. Als dabei zugrundeliegende Zeitlinie, wie sie Knauer als Schnur symbolisiert, wird in der Psychologie das Funktionieren eines isolierten Bereiches der Psyche angesetzt. M. McLuhan 3 hat auf diese Weise die Entstehung des Raumes und des damit verbundenen point of view, der Perspektive, erklärt, daß nämlich der Mensch lemte, ein Sinnesorgan isoliext und unabhängig von allen anderen zu benutzen.

Er sieht im momentanen Erfassen und im sukzessiven Abtasten eines komplexen Wahrnehmungsobjektes nicht zwei verschiedene Techniken, mit deren Hilfe sich die Psyche dem Objekt und dessen Eigenarten angleicht, sondern es sind für ihn die entscheidenden Kriterien

$1 \mathrm{~K}$. Knauer: Die Analyse von Feinstrukturen im sprachlichen Zeitkunstwerk, in: Mathematik und Dichtung, München 1967.

2 A.a.0., s. 193 f.

3 M. McLuhan: The Gutenberg Galarg, Toronto 1962. 
für zwei verschiedene Menschentypen. Dabei entwickelt sich im ProzeB der Menschheitsgeschichte der eine, welcher auf das Abtasten angewiesen ist, zu dem, der simultan erfassen kann. Diese Entwicklung vollzieht sich in der Herausbildung der Fähigkeit zur Separation von Sinnestätigkeiten: point of view ist nur durch die Isolation eines Sinnesorgans und sein unabhängiges Funktionieren möglich. Die entscheidende und auslösende Rolle in diesem ProzeB spielt die Erfindung des Buchdrucks: "Literacy gives people the power to focus a little way in front of an image so that we can take a whole image or picture at a glance. Nonliterate people have no such acquired habit and do not look at objects in our way. Rather they scan objects and images as we do the printed page, segment by segment. Thus they have no detached point of view. They are wholly with the object, they go emphatically into it. The eye is used, not in perspective but tactually, as it were. Euclidean spaces depending on much separation of sight from touch and sound are not known to them." 1

Das sukzessive Abtasten ist also auch beim "literate man" vorhanden, wenn auch ausschlieBlich auf den Fall beschränkt, wo eine bedmuckte Seite gelesen wird. Diese Sonderstellung der Zeichenfolge ist darauf zurückzuführen, daB im sprachlichen Zeichensystem das Hintereinander der Zeichen obligatorisch ist.

Die sprachliche Darstellung ist nicht nur an einen bestimmten zeitlichen Verlauf gebunden, sie besteht aus einer Folge von Zeichen. Das Erleben des Dargestellten wird von einer Folge von Zeichen gesteuert, wobei nicht alle Zeichen, die den sprachlichen Vorgang konstituieren, an der Steuerung gleichermaßen beteiligt sind. Betrachtet man mediale Sukzession und Sukzessivgestalt als die beiden Grundmodi jeder Art von Folge, so unterscheidet sich eine Folge von Zeichen folgendermaßen von einer Folge von Erlebnissen: Dem Strukturcharakter der Psyche entsprechend kann die Alternative, welcher der beiden Modi vorliegt, für jeden konkreten Fall getroffen werden; den Ausschlag gibt der materielle Bestand der Folge (Töne, visuelle Signale usw.). Für das Erzählen ist es charakteristisch, daß für den jeweiligen

1 A.a.0., S.37. 
Sukzessionsfall auch Merkmale des anderen, korrespondierenden Modus zu finden sind. Beim Sprachkunstwerk, das aus einer geschlossenen Sukzession besteht, ist die strukturelle Betrachtungsweise gezmungen, beide Modi mit prinzipiell gleichem Relevanzgrad anzusetzen. Es hänt hier nicht vom Ermessen des Betrachterstandpunktes ab, die Medialität oder Gestalthaftigkeit einer Folge als feststehende, unveränderliche Eigenschaft des Objektes anzusehen, die eigene Perspektive in die Sukzession hineinzuverlegen und sich ihr nachvollziehend anzuschlieBen oder nicht. Vielmehr ist gerade dieser doppelte Aspekt für die Struktur des Erzählwerkes charakteristisch. Der Autor kann dieses Wechselverhältnis gestalten, indem er z.B. den einen Pol als Aufbauschema oder thematisches Modell verwendet, dem anderen durch individuelle Ausformung ästhetische Effekte verleiht.

Der Ansatz einer solchen werkspezifischen Verlaufsform und damit einer werkimmanenten leit ist nötig für die Bestimmung der spezifischen Erscheinungsform des Raumes im Erzählwerk. Die Verbindung der Kategorien von Raum und Zeit ist nicht nur ein SchluBpunkt einer Theorie von der Seinsweise des literarischen Kunstwerkes, sie hat auch ihre Bedeutung für die praktische Analjse. Die eingangs erläuterte geschlossene Einheit zeitlicher Größenordnung in Form des Erzählwerkes wirkt sich im qualitativen Merkmal der Vollständigkeit aus; diese kann sich als Abgeschlossenheit eines Erlebnisses oder einer Handlung, als Vollständigkeit aller Komponenten oder sonstwie auswirken, sie ist in irgendeiner Form mit jedem Erzählwerk verbunden. Als solche Qualität ist sie transponierbar, sie wirkt von der eigentlichen Kategorie, der Zeit, auf die korrespondierende Kategorie, den Raum, hinüber. Zwei Erscheinungsformen dieser Transposition sind dabei zu unterscheiden:

1. Innerhalb eines Erzählwerkes mit geschlossener Zeitstruktur entsteht auch ein vollständiger Raum, auch wenn dieser nur bruchstückhaft, ausschnittweise oder gar nicht sprachlich realisiert wird. Dieses Phänomen bezeichne ich als Raumgenese.

2. Zum anderen kann ein einzelner, akzidenteller Raun, der in den Erzählvorgang als Schauplatz oder mit ornamentalen Funktionen Elngang findet, repräsentativ werden für den allgemeinen Raum mit 
den Eigenschaften der Objektivität, der Allgemeingültigkeit oder der Totalität. Diesen Raum, der fakultativ im Erzählwerk strukturrelevant werden kann, nenne ich den symbolischen Raum.

An diesem Punkt, wo der Raum als universelle Kategorie betrachtet wird, der nur die Zeit ebenbürtig zur Seite steht, ist eine theoretische Betrachtung am ehesten in Gefahr, den Bereich des Erzählens zu verlassen und Grundsätze obligatorisch zu formulieren, die bei der Werkanalyse nicht anwendbar sind. Das Aufstellen dieser Prinzipien würde vom rein erzähltechnischen Sektor wegführen und auf die Intention und Selbstinterpretation einzelner Autoren und literaturgeschichtlicher Epochen eingehen. Eine solche Kunsttheorie würde jedoch den Rahmen der vorliegenden Arbeit sprengen.

Die Raumgenese, auf die ich mich hier beschränke, kann man am besten dann beobachten, wenn man alle räumlichen Motive abstreicht. Räumliche Motive sind all jene Elemente, die den Raum darstellen, etwa eine Figur, die ihn durch ihre typischen Eigenschaften verkörpert, oder ein als Person erscheinender Autor, der über den Raum berichtet, ferner isolierte raumbezogene Erzählphasen an prädestinierten Strukturstellen (Einschub, Exposition u.ä.). Läßt man diese Gegebenheiten außer acht, so erscheint ein ganz anderer Raum als der, welcher zunächst das Objekt des Erzählens zu bilden scheint. Tritt eine solche Figur oder ein solches Thema - zunächst heterogen als Informationsquelle eingesetzt - in den engeren strukturellen Rahmen ein, so erhält dieser Raum auch andere Funktionen oder Eigenschaften. Das sieht man sehr deutlich an den Zeigewörtern, die, ursprünglich nur Hilfssignale des Darstellens, zu Symbolen werden können, wie z.B. in Andrzejewskis "Bramy raju".

Um die Anwendung des Begriffs der Raumgenese in der Analyse anzudeuten, formuliere ich zwei Fragen und versuche, für jedes der im zweiten Teil behandelten Werke einen allgemeinen Ansatzpunkt und Lösungsvorschlag zu finden.

1. Wie kann der Autor, über die Einzeldarstellung hinaus, mit Hilfe eines ganzen Werkes, also im Rahmen der Werkstruktur, den Raum 
künstlerisch gestalten, ihm zu ästhetischer Wirksamkeit verhelfen? 2. Wie trägt der Raum dazu bei, eine Fülle heterogener Gegebenheiten, Zeichen oder diskontinuierlicher Zeitabschnitte, zu etwas Ganzem, Einheitlichem zu machen?

Die nun folgenden Beispiele sollen Möglichkeiten andeuten, wie diese Fragen beantwortet werden können.

1. In "Quo vadis" baut der Uberschuß an Fakten und Details immer wieder den einheitlichen Raum ab und wirkt der Raumgenese entgegen; dadurch wird es möglich, immer neue, überraschende Raumbilder einzuführen, was zur Entstehung eines hinsichtlich der Spannung einheitlichen Zeitabschnittes, der Lesezeit, führt.

2. In "Szkice węglem", ebenfalls von Sienkiewicz, ist der Blick auf den an sich selbstverständlichen Raum eine besondere, problematische Situation. Die damit verbundenen Integrationsschwierigkeiten sind repräsentativ für die allgemeingültige und -menschliche Schwierigkeit, mit neuen Situationen konfrontiert zu sein und mit ihnen fertig zu werden.

3. In der "Lalka" sind das unmotivierte Verhalten der Figuren und die sich daraus ergebenden Entwicklungsabschnitte durch nicht motivierbare Raumvisionen kompensiert. Der Ralm bildet hier eine Art darstellungstechnischer Hilfsebene.

4. In den "Chłopi" ist der Raum als "die Natur" ein praktisch unbegrenztes Gebiet, das zu jeweils neuen Schilderungsansätzen und Bildern den Weg vorzeichnet. Der Raum fungiert als allgemeinste Kategorie, deren Beschreibung nicht erklärt bzw. motiviert werden muß.

Żeromski: Przedwiośnie

5. Erhellung des allgemein menschlich Bedeutsamen als eine Sonderaufgabe wird verbunden mit der qualitativ als höher empfundenen Aufgabe, den Raum zu sehen und zu interpretieren. Der Autor hat sich diese Aufgabe gestellt. Der hier isotrope Raum bietet die Möglichkeit, symmetrische Strukturprinzipien einzusetzen, ohne daß sie einer gesonderten Motivation bedürften.

Dąbrowska: Noce $i$ dnie

6. Kurze, aus dem Zusammenhang herausgelöste Vorgangsausschnitte 
tragen zum schrittweisen Aufbau einer Welt bei. Zeitliche Diskontinuität schafft ein räumliches Kontinuum.

Iwaszkiewicz: Młyn nad Utratą

7. Durch Verlagerung seelischer Zustände in den Raum gelingt es dem Autor, eine lückenhafte Entwicklung auf dem ersteren Sektor zu einer künstlerisch geschlossenen Form zusammenzufügen, mit eigener Verlaufsform und eigenem Höhepunkt.

Schulz: Sklepy cynamonowe

8. Durch die Schaffung ganz eigenartiger und neuer Räume führt der Autor zu unerwarteten Wendungen und Schlüssen auf dem Sektor der Handlungen. Der ästhetische Effekt beruht auf dem unmittelbaren Nebeneinander von kleinsten und gröBten Kategorien. Dieses Nebeneinander dient dem Autor als Konzeption.

Andrzejewski: Bramy raju

9. Die räumliche Symbolik (Liebe als Weg, räumliche Unerreichbarkeit usw.) wird durch die sukzessive Realisierung bei verschiedenen Figuren in stets gleicher Form allgemeingültig. Die räumliche Perspektive wird zur weltanschaulichen.

Zusammenfassend kann man sagen, daß das Erzählen in demselben Sinn eine Vereinheitlichung ist, wie die Wahrnehmung des Raums die Beschränkung auf den Gebrauch eines isolierten Sinnesorganes ist. Der Raum erhält dabei nicht die Eigenschaften dieses Sinnes, so daß man von visuellem oder akustischem Raum sprechen könnte, sonderm er entsteht durch das zeitliche Funktionsschema dieses einen Organs bzw. durch die Zeitstruktur des Erzählens. Von dieser Konzeption aus kann unterschieden werden, ob der Autor sich einem vorgegebenen Erzählmodus und somit einer vorgeprägten Zeitstruktur anschließt, oder ob er isoliert geschaffene Wortkombinationen und -kontexte zusammenfügt und zu einer zeitlichen Größe werden läßt, die erst beim Lesen als solche in Erscheinung tritt. Die übergeordnete Konzeption betrachtet den Raum als Kategorie, die überall ist, wo auch die Kategorie der Zeit da ist; dieses Phänomen bezeichne ich als Raungenese, die nur möglich ist im Rahmen des Erzählens als eines Zeitkunstwerkes. 


\section{B. Anwendungsbeispiele}

1. Inkongruenz von Wortmaterial und Raumerlebnis als Mittel der Spannungserzeugung in Sienkiewicz' "Quo vadis"

Die Struktur des historischen Romans "Quo vadis" von Henryk Sienkiewicz, geschrieben 1895-96, kann vernältnismäBig einfach in Form eines Schemas dargestellt werden. Quellengrundlage und literarische Vorbilder führt Krzyzanowski in seiner Arbeit über Sienkiewicz an. 1 Hier wird auch das Aufbauschema charakterisiert. Historische Grundlage ist die Christenverfolgung unter Nero. Die zwei feindlichen Lager werden nicht durch bekannte historische Persönlichkeiten vertreten, sondern durch Randfiguren; es gibt einen positiven und einen negativen Helden, die auch in einer Liebesaffäre als Rivalen auftauchen. Der negative Held muß nach den verschiedensten Abenteuern unterliegen. ther dieses stereotype Schema hinaus beruht der literarische Effekt in folgendem: "Indem er nämlich die in seiner Phantasie entstandenen Helden mit farbigen Geschichtsereignissen verbindet, versteht es Sienkiewicz, trotz der Anwendung stets eines und desselben, nur entsprechend modifizierten Strukturschemas die Neugier des Lesers in ständiger Anspannung $z u$ halten, sie mit immer neuen Abenteuern zu beleben, die er mit geradezu unerschöpflicher Erfindungsgabe anhäuft." (S. 215) "Das Resultat dieser Geschichtskonstruktion ist eine ungewöhnlich reiche Skala ästhetischer Effekte." (S. 219) Der K ampf des Guten gegen das Böse wird auf historischer Bühne ausgetragen und endet siegreich für das Gute.

Der Schauplatz dieses Kampfes ist das Rom der Regierungszeit Neros. An den dramatischen Geschehnissen sind nicht nur die zwei Hauptkontrahenten beteiligt, sondern noch eine Reihe anderer Figuren, so daß sich eine Vielzahl von Handlungseinheiten und dramatischen Zuspitzungen ergibt. Der Leser wird also in einen ihm völlig unbekannten Raum versetzt; und das ist dem Autor, wie der große Erfolg dieses Romans zeigt, gut gelungen. Um seine Technik zu untersuchen, greife ich eine Erzählphase heraus, die den Gang des Helden Vinicius und seines Gehilfen Chilon zum Ostrianum,

$1 \mathrm{~J}$. Krzyżanowski: Henryk Sienkiewicz, in: Obraz literatury polskiej 4/2, Warszawa 1966, S. 201-240. 
einem auBerhalb Roms liegenden Friedhof, der den Christen als Iokal für thre nächtlichen Zusammenkünfte dient.

"Sie gingen durch den Vicus Patricius, den Viminal entlang zum früheren Viminalischen Tor, neben der Ebene, auf welcher Diocletian später die herrlichen Thermen erbaute. An den tberresten der Mauer des Servius Tullius vorbei gingen sie über schon fast leere Plätze und gelangten zur Via Nomentana, dort aber bogen sie nach links ab zur Via Salaria und befanden sich unter Hügeln voller Sandgruben, hie und da auch Grabstätten. Mittlerweise war es auch schon vollständig dunkel geworden, und da der Mond noch nicht aufgegangen war, fiel es ihnen nicht leicht, den Weg zu finden, wenn nicht, wie Chilon es vorausgesagt hatte, die Christen ihn selber gezeigt hätten. Rechts und links und vorn waren dunkle Gestalten zu sehen, die vorsichtig den sandigen Schluchten zustrebten. Manche von diesen Leuten trugen Laternen, verdeckten sie jedoch nach Möglichkeit mit den Mänteln, andere, die den Weg besser kannten, gingen im Dunkeln. Das geübte Soldatenauge des Vinjc ius unterschied nach den Bewegungen die jüngeren Männer von den Greisen, die sich auf Stöcke gestützt dahinschleppten und von den Frauen, die sorgfältig in lange Stolen gehüllt waren." 1

Es sind also folgende Details, die dem Leser den für ihn neuen Schauplatz charakterisieren: Vicus Patricius, Viminal, Viminalisches Tor, Thermen des Diocletian, Mauer des Servius Tullius, Via Nomentana, Via Salaria, Ostrianum; ferner Hügel, Sandgmuben, Grabstätten, sandige Schluchten, der Mond, dunkle Gestalten und Laternen. Der Autor übernimmt die Rolle des ortskundigen Kommentators, der den Leser als Beobachter der Figuren bei ihrem abenteuerlichen Gang räumlich orientiert und auf Besonderheiten aufmerksam macht.

Ist es Aufgabe der literaturwissenschaftlichen Raumanalyse, mit Hilfe dieser Angaben und durch Vergleich mit den historischen Stätten diesen Raum zu rekonstruieren? Es wäre ein schwieriges Problem, die genaue Position der angeführten Baudenkmäler festzulegen, unmöglich, ein genaues und anschauliches Bild der durch-

1 H. Sienkiewicz: Quo vadis, Krakau 1924, S. 158. 
messenen Ortlichkeiten zu geben.

Der Autor tut nichts, um dem Leser diese Rekonstruktion zu erleichtern. Im Gegenteil: Gerade die bevorzugte Verwendung lateinischer Termini, deren Bedeutung dem Leser unklar bleibt und mit denen er auf keinen Fall eine bestimmte Anschauung verbindet, läBt kein Erlebnis eines bestimmten Raumes entstehen, den man auf sich wirken lassen kann und auf welchem die Figuren sich bewegen. Das Ziel dieser Technik ist klar: Da kein Raum da ist, der dem Leser als objekt vorliegt, in dem er sich orientieren kann, wird das Raumerlebnis zeitlich eingegrenzt und in die spezielle Lesezeit hineinverlagert. Der Leser hat keine sichere Distanz von dieser Szene, die allein inm eine genaue und nachvollziehbare Anschauung der Positionsangaben und Details geben könnte. Indem ihm die Möglichkeit genommen wird, inne zu halten und sich einen den Zeichen adäquaten Raum vorzustellen, entsteht für ihn eine zeitliche Phase, in der er sich staunend mit einem ungewöhnlichen Raum konfrontiert sieht. Es handelt sich also um eine Sukzessivgestalt, deren Qualität in der Hervorrufung einer unheimlichen Stimmung liegt.

Wenden wir den Blick noch einmal auf die einzelnen Raumelemente. Die Bezeichnung der einzelnen Orte erfolgt auf Lateinisch, sie sagen über die Beschaffenheit der Gegebenheiten nichts aus, werden aber als selbstverständlich vorausgesetzt und klingen fremdartig. Die herrlichen Thermen sind zum geschilderten Zeitpunkt noch nicht da, wirken aber genauso stark wie die schon anwesenden Bauwerke. Ein gleiches gilt vom Mond, der der Situation ein unheimliches Licht verleiht, ohne aufgegangen zu sein. Die genauen Positionsangaben, links, rechts und vorne, hier und dort, geben dem Leser das Gefühl, dabei zu sein, obwohl für den analysierenden Beobachter die Szenerie durch sie ihre feste, nachprüfbare Gestalt verliert. Sie entstammen einem Bezugssystem, das um den Beobachterstandpunkt zentriert ist, und nicht einer standpunktsunabhängigen Festlegung räumlicher Relationen. Alle Gegenstände haben jedoch die Fähigkeit, eine intensive räumliche Wirkung aufrecht zu erhalten und fortzusetzen. In dem Maße, wie durch das Fortschreiten des raumbezogenen Beschreibungsvorgangs die in einem Erlebnis resultierende Wirkung sich verstärkt, nimmt die räumlich anordnende Komponente des Details ab. Je mehr eindrucks- 
volle Einzelheiten erwähnt werden und ihre Vielfalt sich zu einer einheitlichen Wirkung zusammenschlieBt, desto geringer wird die Möglichkeit und desto kleiner die Berechtigung, sie in ihrer spezifisch räumlichen Position festzulegen und eine verbindliche Anordnung vorzunehmen.

In welcher Umgebung steht ein solcher Erzählabschnitt? Wie ist er in den Verlauf der dramatischen Ereignisse eingeordnet, gibt es in ihm eine bestimmte Zäsur, die eine Ablenkung der Aufmerksamkeit auf die räumliche Umgebung gestattet, ohne daB deren Erlebnis einer ungestörten Beobachtung des konsequenten Handlungsverlaufs Abbruch tut? Zur Erhellung dieser Frage greife ich ein anderes Beispiel heraus, die Darstellung von Neros Prachtgärten. Diese Darstellung erfolgt aus der Sicht Ligias, und zwar zu einem Zeitpunkt, da sie in höchster Gefahr schwebt, aber mit einer Ausführlichkeit und Ruhe, wie man sie in einer solchen Situation nicht erwarten kann.

"Ligia sah zum ersten Mial im Leben diese herrlichen Gärten voller Zypressen, Pinien, Eichen, Olbäume und Myrten, zwischen denen ein ganzes Heer von Statuen weiB schimmerte, die ruhigen Flächen der Teiche glänzten, wo ganze Haine von Rosen blühten, vom Wasserstaub der Springbrunnen befeuchtet, wo die Eingänge zu zauberhaften Grotten von Efeu und Heinreben überzogen waren, wo auf den Wassern silberweiBe Schwäne schwammen und zwischen den Statuen und Bäumen zahme Gazellen aus Afrika umhersprangen und eine bunte Vogelschar, aus allen bekannten Iändern der Irde herbeigeholt." (S. 80)

Diese Ausführlichkeit zu diesem Zeitpunkt zeigt, daB der Auto: nicht in einer konzeptionellen Trennung von Szenerie und Handiung sein Ziel sieht, sondern im Aneinanderfügen wirkungsmäßig gleichstarker Erlebnisphasen. Die Integration erfolgt nicht durch eine Ablösung von statischen, raumbezogenen und dynamischen, handlungsfortführenden Phasen, sondern durch die Auf rechterhaltung einer bestimmten Erlebnisintensität, gleichgültig, ob sie durch eine ungewöhnliche Ortlichkeit oder ein ungewöhnliches Ereignis he:vorgerufen wird. Bei der Handlung ist es das Element der Spannung, beim Raum das der Exotik, und es sind nicht spezifisch 
räumliche Elemente, die den Blick auf das Letzere, und nicht hand lungsimmanente Kriterien, die den Blick auf das eine oder das andere lenken.

Der Leser wird also auf diesem historischen Schauplatz nicht orientiert, ausschlaggebend sind für sein Erlebnis aber die Komponenten der Exotik, der Mosaikhaftigkeit, der bunten und nicht alltäglichen Vielfalt und Phantastik. Diese Nomente steuern den Verlauf des Erlebens. Bei einem dieser räumlichen Bilder innezuhalten und sich Gedanken darüber zu machen, wie dieser Raum aufgebaut ist, oder sich seine szenische Gestalt zu vergegenwärtigen, wird also nicht die Freilegung eines Strukturzugs zum Ergebnis haben, sondern dem vom Autor beabsichtigten Effekt widersprechen. Entweder wird man den dichten Ablauf der Erzählphasen unterbrechen und die darauf beruhende Spannung zunichte machen, oder man erhielte eine Konstruktion, die in den gebotenen Fakten nicht verbindlich enthalten ist und von einem anderen Leser in ganz anderer Form realisiert werden kann. In diesem Sinne ist die Konzentration auktoralen Gestaltens auf die zeitlichen Relationen gerichtet, auf die Anordnung und tberschneidung von irlebnisphasen auf seiten des Lesers. Indem dieser außerstand gesetzt wird, sich mit den gebotenen Fakten wie mit Informationen auseinanderzusetzen und sich in diesem Raum reflektierend zu orientieren, wird er gezwungen, weiterzulesen. Ein lückenloses und atemloses Lesen ist die Voraussetzung für die ästhetische Wirkung dieses Romans.

Der Leser sieht sich in einen fremden Raum versetzt. Aber es ist kein für ihn ganz und gar unbekannter Raum. Vielmehr liegen Raumtopoi vor, die in starkem Maße das beinhalten, was der Durchschnittsleser beim jeweiligen Stand der Dinge erwartet. Er findet hier das, was inm unter dem Wort Rom bekannt ist bzw. welche unreflektierten Vorstellungen er damit verbindet, zu einem Schauplatz zusammengefügt. Würde ein solcher Leser genötigt, diesen Vorstellungsgehalt deutlich zu machen, zu beschreiben oder zu rekoastruieren, so wäre er dazu nicht imstande. Sienkiewicz'Leistung ist es nun, nicht zu analysieren, was an diesen common-senseVorstellungen richtig ist bzw. was eigentlich historisch wirklich 
vorgelegen hat, sondern diesen unbedachten Gegenheiten zum Leben und $z u$ Aktualität $z u$ verhelfen. In unserem letzten Beispiel, dem gefährlichen Gang des Helden auf der Suche nach der verlorengegangenen Geliebten, findet man die Requisiten des gefährlichen Ortes, Gräber, Schluchten, Dunkelheit; im zweiten Beispiel die Merkmale des prächtigen Ortes, Springbrunnen, Grotten, Schwäne usw. Daß das Staunenswerte vor dem konisret Beobachteten steht, zeigt die Anmerkung, daß die bunte Vogelschar aus allen bekannten Ländern der Erde herbeigeholt wurde. 
2. Merkmale des Raumes und der Charakteristik der Figur in Sienkiewcz "Szkice ręglem" und Prus' "Lalka"

Die in "Quo vadis" vorgefundene Raumfiktion zeigt, daß dem Raum ein gewisser kigenwert zukommt, das heißt, er dient nicht einem bestimmten Zweck, etwa der Orientierung des Lesers und als Verständnishilfe für die verwickelten Handlungen, sondern seine ornamentalen Funktionen stehen vor seiner nachprüfbaren szenischen Struktur zurück. Im folgenden Kapitel analysiere ich einige Beispiele, in denen die Schaffung von Typen im Vordergrund steht und die Fiktion des Raumes diesem Zweck untergeordnet ist. Gemeinsam ist den betreffenden Typen, dem Schreiber Zołzikiewicz und der Bäuerin Rzepowa aus Sienkiewicz" "Szkice Węglem", sowie den Figuren Wokulski, Izabella, Rzecki und Eęcki aus Prus' "Lalka" ein fester Stellenwert in einer bestimmten sozialen Schicht, die Zugehörigkeit zu einem gesellschaftlich fixierten Stand. Die Rolle und Dominanz dieser Zugehörigkeit innerhalb der Charaktere ist verschieden, aber in allen Fällen wird sie als eine problematische Beschränkung empfunden, teils vom Autor, teils von den Figuren selbst. Wie diese auktorale Perspektive sich zur figuralen verhält, soll an diesen Beispielen untersucht werden.

Zunächst formuliere ich noch einmal die s'rage nach der Uberschneidung verschiedener Perspektiven. Der Darstellende ist in jedem Fall der Autor; er ist somit auch verantwortlich für den Blickwinkel, aus dem der Raum gesehen und erlebt wird, für die Wortwahl, die bei seiner Darstellung Verwendung findet, und für den Grad der Angemessenheit der zu diesem Zweck ausgewählten Zeichen. Doch kommt gerade in der Lalka hinter diesen Darstellungen ganz deutlich die rigur zum Vorschein. Eigentlich ist sie es, die in diesem Raum ist, ihn erlebt und sich bemüht, ihn mit ihren eigenen Worten zu erfassen und darzustellen. Wie können diese beiden Funktionen und Beiträge, von seiten des Autors und der Figur, begrifflich geschieden werden? Wie sind beide Perspektiven zu einem einheitlichen Erzänlvorgang koordiniert?

Das Beispiel einer flachen Charakteristik bietet die Frau Rzepowa in der Novelle "Szkice Węglem" von H. Sienkiewicz, geschrieben 
1877. Sie ist eine einfache, tüchtige Bäuerin, jung und hübsch, aber $z u$ beschränkt, um den Nachstellungen und den Intrigen des Schreibers Zołzikiewicz gewachsen zu sein. Ihre Position in dramatischen Konflikt beleuchtet der Autor durch eine kleine Szene in der Kirche, wohin sie in ihrer Ausweglosigkeit hilfesuchend gelaufen ist. "Es schien ihr, als ob die Engel des Himmels sie von der Erde aufhöben wie ein armseliges Blatt, bis zum Himmel, wo es keinen Herrn Zołzikiewicz gibt und keinen Schulzen, auch keine Militärausschreibung ..." 1 Das Erlebnis des Emporschwebens ist auf negative ileise beschrieben: nicht die Vision von überirdischen Dingen charakterisiert die Situation, sondern der Wegfall der Elemente, die das Erdendasein der Figur bestimmen. Dieser negative Zug und die damit erzielte ironische Wirkung ist charakteristisch für diese Novelle. Auch der Schauplatz erhält diesen Zug durch die sprechenden Namen der Ortschaften; Barania Głowa heiBt Schafskopf und Osłowice heiBt etwa Eselsstadt. Eine höhere, das dinglich Gegebene übersteigende Sphäre wird immer wieder transparent, gelangt aber nie ganz zur erwarteten Realisierung oder wird durch den Kontrast mit banalen Dingen in ein groteskes Licht gerückt. Dies geschieht z.B. im Gespräch von zwei jungen Leuten aus dem Gutsbesitzerstand, welche durch das Dorf spazieren gehen. "Zwischen diesen Hütten, neben den Dorfkindern, den Bauern und der groben Umgebung sahen die beiden aus wie irgendwelche Wesen von einem anderen Planeten. Wie angenehm war es zu denken, daß überhaupt kein Band zwischen diesem stolzen, blühenden und poetischen Paar und dem prosaischen, animalischen Dasein des Dorfes voll grauer Realität vorhanden war. Es bestand kein Band, zumindest kein geistiges." (S. 75) Uber den Inhalt des erwähnten Gesprächs erfährt man nichts; das Wesen der behandelten Themen ist in folgendes Bild gekleidet: "Von Buch zu Buch hüpften sie, wie ein Schmetterling von Blüte zu Blüte hüpft ... So ein Gespräch schwebt wie ein Vogel zu den Bergen, in blaue Sphären ..." (S. 75 f.) Je mehr die Isolation der Figuren von ihrer Umgebung betont und unterstrichen wird, umso deutlicher verschmilzt beides zu einer mittels des Lesevorgangs anschaulichen, vorstellbaren Situation.

1 H. Sienkiewicz, Nowele wybrane, Warszawa 1949, S. 71 f. 
Am deutlichsten wird in dieser Novelle der Zusammenhang von Eigenschaften der den Raum erfüllenden Gegenstände und dem Typ der Figur bei der Schilderung der Wohnung des Helden Zołzikiewicz. Das Zimmer sieht so aus: "Es stand da ein fast nie gemachtes Bett, auf dem Bett zwei Kissen ohne tberzug, welchem die Federn ausgingen; daneben ein Stuhl, auf diesem ein Tinten$f a B$, eine Feder, Kanzleibücher, einige Hefte der "Isabella aus Spanien" in der Ausgabe von Herrn Breslauer; zwei schmutzige englische Stehkrägen, ein Glas Pomade, Zigarettenhülsen und schließlich auf einem Blechleuchter eine Kerze mit rotem Docht und mit Mücken, die im Wachs um den Docht herum versunken waren. Beim Fenster hingen einige Spiegel, gegenüber dem Fenster aber war die Kommode aufgestellt, welche die überaus elegante Toilette des Herrn Schreibers enthielt: Kniehosen in den verschiedensten Schattierungen, Westen in märchenhaften Farben, Krawatten, Handschuhe, Lackstiefel, ja sogar ein zjlinder, den der Herr Schreiber dann benutzte, wenn ein Gang in die Kreisstadt 0słowice anfiel." (S. 29) Daß der Bewohner dieses Zimmers ein eingebildeter Geck ist, der sich nur deshalb durchsetzt, weil seine sfitmenschen noch dümmer sind als er selbst bzw. weil er mehr aus sich macht, als er ist, wird dem Leser schon vorher klar, schon beim Lesen der ersten Zeilen. Hier verdichtet sich der Eindruck nochmals zu größter anschaulicher Intensität, und $z$ war mit Hilfe der spezifisch räumlichen Elemente dieses Zimmers. Hier herrscht peinliche Genauigkeit bei Angaben von Positionen: Auf dem Bett, daneben ein Stuhl, auf ihm ein TintenfaB, auf ihm ein Blechleuchter, im Wachs um den Docht, beim Fenster, gegenüber dem Fenster. Diese peinliche Genauigkeit, welche doch dem Leser zur anschaulichen Realisation dieses Zimmers nicht nötig ist, spricht lebendig für die Beschränktheit und Pedanterie des Bewohners. Trotz der angestrebten Vollständigkeit in der Erfassung aller Gegenstände sind es fast nur Kleidungsstücke, die ihren Herrn repräsentieren.

Einer ähnlichen Technik bedient sich B. Prus in der "Lalka"; der Handlungsgehilfe Rzecki, eine Randfigur, die immer wieder dazu dient, das zentrale Romangeschehen aus einer unbeteiligten Perspektive zu betrachten, ist ebenfalls durch seine Behausung cha- 
rakterisiert. Hier ist es aber nicht wie oben eine bestimmte Klasse von Gegenständen, die das Wesen des Bewohners beleuchten, sondern es sind die normalen Requisiten eines Zimmers wie Bett, Tisch, Stuhl, Schrank. Sie haben alle die gleiche Eigenschaft, die Altertümlichkeit, wie auch diese Figur, welche ihre Glanzzeit als Offizier der ungarischen Armee und als Abenteurer schon hinter sich hat. "Ein Messingleuchter für Talgkerzen, die niemand mehr anzündete, und eine Stahlschere, mit der schon niemand mehr einen Docht abschnitt ... Herr Ignacy wohnte seit 25 Jahren im Zimmerchen beim Geschäft. Im Laufe dieser Zeit wechselte das Geschäft die Besitzer und den Fußboden, die Schränke und die Fensterscheiben, den Bereich seiner Tätigkeit und die Gehilfen; aber das Zimmerchen des Herrn Rzecki blieb immer dasselbe. Es war in ihm das gleiche traurige Fenster, welches auf denselben Hof ging, mit demselben Gitter, auf dessen Sprossen sich eine Spinnwebe von vielleicht einem Vierteljahrhundert festgehängt hatte, und mit einem Vorhang von ganz bestimmt einem Vierteljahrhundert, welcher einmal grün gewesen war, jetzt aber verschossen war vor Sehnsucht nach der Sonne." 1 War es im ersten Beispiel die mosaikartige Ansammlung von Details, die Zeugnis von der Figur ablegten, so ist hier ein einzelner Zug, der alle Gegenstände umfaßt, bezeichnend für den Bewohner und trifft eine Schicht seiner Persönlichkeit. Rzecki ist ja nicht nur ein altertiumliches Requisit aus der Vergangenheit. Er nimmt am ganzen Handlungsverlauf und an allen wichtigen Geschehnissen stets lebhaften Anteil, obwohl er in einem Zimmer wohnt, "welches hinsichtlich seiner länge und der in ihm herrschenden Dunkelheit einem Grab ähnlicher ist als einer Wohnung." (S. 11) Flache Charakteristik als die Erfassung nur eines Zuges der Persönlichkeit bedeutet in diesem Fall: Der die Figur umgebende Raum ist von Gegenständen nur einer Klasse angefüllt bzw. alle diese Gegenstände haben die gleiche Eigenschaft.

Das Ergebnis der Gegenüberstellung von Sienkiewicz und Prus ist folgendes: In beiden Fällen ist die Fiktion eines Typs Hauptanliegen des Autors. Die Beschreibung des Raumes, in welchem die

1 Bolesław Prus: Lalka, Bd 1-2, Warszawa 1949, s. 11. 
beiden Typen wohnen, ist eine Technik, den betreffenden Charakterzug zu beleuchten. In der Lalka tritt dieser Erzählabschnitt stärker aus dem Rahmen der übrigen Erzählung heraus, da Rzecki als eine Zentralfigur des dramatischen Geschehens noch andere Rollen spielt als die, welche durch die Beschreibung seines Zimmers umrissen wird. Er ist also nicht nur ein alter Mann, der die Vergangenheit verkörpert, ohne lebendige Anteilnahme an den um ihn her stattfindenden Ereignissen. Zołzikiewicz ist, der Kürze der Erzählung entsprechend, ein flacher Charakter, und somit ist die Beschaffenheit seines Wohnzimmers eindeutiger festgelegt.

Beide Autoren benützen für ihr Ziel, einen ihnen vorschwebenden Typ zu realisieren, verschiedene Wege. Sienkiewicz sammelt verschiedene Gegenstände, die charakteristisch für ihn sind. Diese

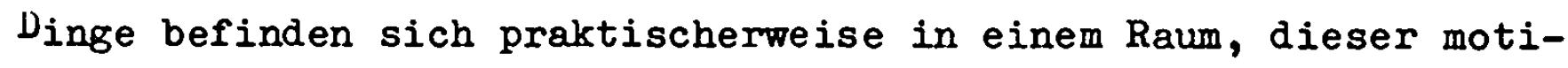
viert die Ansammlung, verschwindet aber wieder, sobald die Handlung weitergeht. Der Held bleibt, nicht der Raurn. Prus sucht den Raum seiner Figur auf und betrachtet ihn als solchen. Eine Eigenschaft dieses Raumes ist auch die der Figur, des Handlungsgehilfen Rzecki. Letzterer hat freilich auch noch andere Eigenschaften und Charakterzüge, und diese Seiten seiner Persönlichkeit treten zutage, sobald er seine grabähnliche Kammer verläßt.

Der Autor verleiht einer Figur eine bestimmte Eigenschaft, indem er diese am sie umgebenden Raum demonstriert. Einer gleichen Technik, wenn auch mit umgekehrten Vorzeichen bedient sich Prus bei der Charakteristik der Eęckis, einer sozial absteigenden Adelsfamilie.

Das Interieur ihrer Wohnung wird folgendermaßen beschrieben: "Jedes Zimmer war, ganz nach Maßgabe der Notwendigkeit, mit anderen verbunden oder bildete eine in sich geschlossene Einheit. Möbel gab es schlieblich in ausreichender Anzahl, weder zu wenig, noch zu viel, und jedes Stück zeichnete sich eher durch behagliche Einfachbeit aus als durch ins Auge springenden Zierat." ( S. 53) "Als er (Wokulski) auf den Hausherrn wartete, langweilte er sich nicht etwa, denn es umgaben ihn ja Dinge, die der Betrachtung wert waren. Gleichzeitig versetzte ihn der Anblick der Gegen- 
stände, die nicht gestern gemacht waren und noch einigen Generationen dienen konnten, in eine Art feierliche Ditimmung! " (S. 56)

Der Effekt beruht im übertriebenen und ausschlieBlichen Streben des Autors nach Präzision in der Erfassung und Beobachtung der räumlichen Gegebenheiten. Dadurch wird jedoch dieser Raum nicht plastischer, sondern versinkt in farblose Unanschaulichkeit, wobei jedoch der Leser sogleich erfaBt, was für eine Art von Interieur hier gemeint ist. Das Bild einer unerschütterlichen Ordnung scheint sich hier zu bieten, doch gerade auf Grund der tberbetonung des Ordnungsmoments bedarf es nur eines winzigen Details, um sie aus den Angeln zu heben: "... und nach einer Weile kehrte er zurück mit einem Brief auf einem silbernen, vielleicht aber auch nur versilberten Tablett." (S. 81) Präzisierung als zielgerichtete Bedeutungserfüllung ist unterbrochen und wird nicht zuendegeführt.

Von welcher Art sind die Leute, die in solchen Räumen wohnen? Mit dem Schematismus des Raumaufbaus geht der Verlust der privaten Atmosphäre Hand in Hand. Zwar nehmen die Bewohner dieser Räumlichkeit im weiteren Verlauf des Romangeschehens durchaus lebendigen Anteil an seelischen Konflikten, doch ihr wesentlicher Charakterzug ist durch diese :iohnung festgelegt; es ist die ständige Gefahr des Abgleitens in die Leblosigkeit, der ständige Kampf um die Behauptung der eigenen Individualität.

Dies beleuchtet der Autor auch in den Sätzen, mit welchen er Isabella, eine der Hauptfiguren, einführt. "Alles an ihr war originell und ausgezeichnet, inr Wuchs größer als mittel, eine sehr wohl gestaltete Figur, üppiges blondes Haar mit einer aschgrauen Nuance, eine gerade Nase, der Mund etwas zurückgezogen, Zähne wie Perlen, Hände und Füße wie modelliert ..." (S. 56)

Ist es hier der Autor, welcher seine Figuren durch diese Bilder charakterisiert, so finden sich immer wieder Stellen, wo die Figur ihre eigene Situation in Form eines räumlichen Bildes vor sich sieht. Das Verhältnis von Isabella zum Helden Wokulski schwankt bis zum Schluß des Romans zwischen Liebe und Abneigung. Sie hat immer wieder Visionen, in denen ihr diese zwiespältige Lage in Form eines räumlichen Bildes zum Bewnßtsein kommt, etwa 
durch einen Traum. "Es schien ihr, als fahre sie mit dem Wagen durch eine ihr unbekannte Gegend. Die Iandschaft ist einem riesigen Ring aus Wäldern und grünen Bergen ähnlich, und ihr Wagen befindet sich am Rand dieses Ringes und fährt ins Tal." (S. 86 f.) In der Niederung sieht sie dann Dampf und Rauch, daraus steigt die Person Wokulski's zu ihr herauf.

In diesem Bild wird nicht die Art des Verhältnisses zwischen diesen beiden Figuren vor den Augen des Iesers als eines Zuschauers demonstriert. Vielmehr findet der Versuch der Figur, sich über die eigene Situation klarzuwerden, in den Kategorien räumlichen Ërlebens statt. Nie sind es Räume, die die Figur tatsächlich und gegenständlich vor sich sieht, welche ihr in Form des Schauplatzes Warschau real vorliegen, sondern es finden Visionen statt, welche die schauplatzmäßigen Funktionen des Raumes, die Bühne und den Standpunkt auf ihr, übersteigen.

Es folst ein weiteres Bild, mit dem Prus das Verhältnis Isabellas zu Wokulski charakterisiert: "Er (Wokulski) war auch niemandem ähnlich, und wenn man ihn überhaupt mit etwas vergleichen konnte, so sicherlich mit einer Gegend, durch welche man den ganzen Tag läuft, und wo man Ebenen antrifft und Berge, Wälder und Wiesen, Wasser und Wüste, Dörfer und Städte, und wo noch, außer den Nebeln des Horizonts, unklare Erscheinungen auftauchen, die schon keiner bekannten Sache mehr ähnlich sehen." (S. 316)

Was leistet der Autor bei diesen Erzählphasen? Er fügt eine Anzahl von Elementen zusarumen, die in ihrer Kombination ein räumliches Bild ergeben. Die Auswahl der Elemente erfolgt nach dem Gesichtspunkt ihrer Fähigkeit, sich problemlos zu einem Raum zu vereinigen, sei es zu einem Interieur, einer Landschaft oder einer sonstigen Szenerie. Die Natur dieses Raumes für sich betrachtet wirft noch kein Licht auf den Sinn, welchen er verdeutlichen soll. Diesen machen erst die Zeichen transparent, die als Vertreter für diese Elemente fungieren. Und diese Bezeichnunjen entstammen dem sprachlichen Horizont der Figuren. Der Figur fällt also die Aufgabe zu, für den Raum die adäquaten Wörter zu finden, und diese symbolisieren durch ihr Verhältnis $z$ den realen Gegebenheiten deren inneren Zustand. Indem die Darstellung aus der Figurenper- 
spektive erfolgt, geben diese einen Einblick in ibre seelische Verfassung, und diese motiviert dann den weiteren Verlauf der Handlung. Der Entrurf des Raumbildes geschieht also aus der übergeordneten Perspektive des Autors, die Auswahl der Zeichen aus der Perspektive der Figuren, deren Bemühen um die Formulierung ihres Erlebnisses auf indirektem Weg Darstellungsziel ist. Der Raum selber ist durch keine Besonderheit gekennzeichnet, die thn darstellenswert machen würde.

Dieser doppelten Perspektive entsprechend erfolgt die Integration dieser Erzählphasen auf zweifache Weise. Der Autor unterbricht den normalen Handlungsverlauf und lenkt den Blick in einem Zeitpunkt auf den Raum, zu dem keine dramatische Konsequenz eine Darstellung des Raumes erfordert. Von den Figuren aus gesehen erfolgt dieser Blick auf den Raum nicht in einer Situation, wo ihr handlungsbedingter Standort von einer außergewöhnlichen Position im Raum bestimmt ist. Vielmehr erfolgen all diese Erlebnisse in Form von Visionen und Träumen, die vor den Augen der Piguren aufsteigen und denen sie mit ihren eigenen Worten Ausdruck verleihen wollen.

Diese Visionen stehen nicht nur im Handlungsverlauf isoliert da und unterbrechen dessen dramatische Folge, sie übersteigen auch den Horizont der Figuren, gemessen an Ihren sprachlichen ÄuBerungen im "normalen" Geschehen, sei es in alltäglichen Situationen oder in dramatisch bewegten Konflikten, sie stehen im Widerspruch zu ihrem Charakter und ihrer Persönlichkeit. Das hat auch die zeitgenössische Kritik Prus angekreidet, wie J. KułczJcka-Saloni berichtet: "Ein oft anzutreffender Vorwurf ist die Behauptung, der Pigur des Helden fehle es an Wahrscheinlichkeit psychologischer Art, nämlich daB Prus ibn mit widersprüchlichen Eigenschaften bedacht hat: Neben eiserner Energie, Rauheit und Standhaftigkeit im Vorgehen befahl er ihm liebesbedürtig zu sein und sentimental wie ein Schüler." 1 Diese Ungereimtheiten seiner Charaktere und die Isolation der räumlichen Visionen innerhalb des dramatischen Vorgangs hat Prus dadurch zu struktureller Geschlossen-

1 KuYczycka-Saloni, J.: BoleaIaw Prus, in: Obraz literatury polskiej 4/2, Warschau 1966, S. 166. 
heit zusammengefügt, daß er das Erstaunen darüber einer Randfigur des Geschehens selber in den Mund legt und sie so dem Romanganzen integriert. Das 18. Kapitel ist überschrieben: "Erstaunen, Vorausblicke und Beobachtungen des alten Untergebenen." Dort heiBt es, und diese Stelle ist nur stellvertretend für eine ganze Reihe von Kapiteln, in welchen durch diese Figur aus der Froschperspektive ein Gegengewicht zu den Ungereimtheiten und Sprüngen in der Entwicklung der Hauptfiguren geschaffen wird: "Für Herrn Ignacy. Rzecki kam wieder eine Epoche der Beunruhigungen und des Staunens. Dieser selbe Wokulski, der ein Jahr zuvor nach Bulgarien geeilt war, sich vor einigen Wochen wie ein Magnat mit Wettrennen und Duellen vergnügt hatte, dieser selbe Wokulski fand heute einen ungewöhnlichen Geschmack an Theateraufführungen." (S. 385)

Der den Sinnen sich bietende reale, dingliche Raum wird also für die Figur in einer bestimmten Situation plötzlich als solcher sichtbar, und es erscheint hinter den raumfüllenden Gegenständen eine andere Realität, welche für einen Augenblick einen sinnhaften Zusammenhang demonstriert. Wokulski sitzt beispielsweise auf der Tribüne in einer sehr negativen Verfassung, an einem stimmungsmäßigen Tiefpunkt, und wartet auf den Beginn des Pferderennens. "Die Frauen schienen ihm häBlich, ihre farbigen Kleider wild, ihre Koketterie widerlich. Die Männer waren dumm, die Menge ordinär, die Musik lärmend. Als er zur Galerie hinaufging, lachte er über die knarrenden Treppen und die alten Wände, an welchen Spuren des eindringenden Regens zu erkennen waren." (S. 288) Das ist die sinnlich gegebene Realität, der Raun als Komplex von Wahrnehmungsgegenständen. Der Wahrnehmungsakt der Figur wird unterbrochen, die Vision setzt ein, wobei nicht die Gegenstände transparent werden, sondern das Wahrnehmungsfeld thematisch umstrukturiert wird. "Was tun diese Galerien das ganze Jahr über? dachte er. Und er träumte, daß auf den zu Staub zerfallenen Bänken jede Nacht die verstorbenen Bankrotteure sitzen, büBende Kokotten, Hohlköpfe aller Art und Verlierer, die man sogar aus der Hölle vertrieben hat und die nun beim traurigen Schein der Sterne Wettläufe von Skeletten der Pferde betrachten, die auf dieser Bahn zugrundegegangen sind. Es schien ihm sogar, daß er in diesem 
Moment die vermoderten Kostüme vor sich sähe und den Geruch des Moders verspüre. Der Schrei der Menge weckte ihn." (S. 289)

Hier liegt ein Höhepunkt der erlebnismäBigen Intensität vor, der nicht mit einem Höhepunkt im konfliktgebundenen Verlauf der Handlung übereinstimmt. Durch inn wird eine Entwicklungsphase des Helden beleuchtet und dieser Vorgang ist verbunden mit dem kurvenförmigen Anstieg der Relevanz räumlicher Wirkungen und Erlebnisse für die Struktur dieses Romans.

Im theoretischen Teil steht die Raumfiktion nach der Raumdarstellung, als ihre Fortsetzung und Weiterentwicklung durch den Einsatz von diffizileren sprachlichen Mitteln, mit Ergebnissen, in denen die sprachlichen Eigenarten und Gestaltungsmomente überwiegen. Bei Prus zeigt sich hier eine andere Hierarchie. Der Autor fingiert einen Raum, doch ist das dadurch entstandene Objekt Ziel der Erzählung und ästhetischer Selbstzweck. Erst dadurch, daB die Figur diesen Raum darzustellen versucht im Sinn der Auswahl adäquater Zeichen (siehe oben Kap. 3), tritt er vor das Auge des Lesers, wobei das räumliche Erlebnis überlagert wird vom seelischen Konflikt der Figur, den der Leser erst hier in seiner ganzen Problematik kennenlernt. Dieser Konflikt ist gekennzeichnet durch den Versuch der Figur, ihren eigenen Standort, der seine Grundlagen in einer gesellschaftlichen Schicht verloren hat, neu zu finden. Der Höhepunkt dieses Konfliktes ist beim Helden Wokulski verwirklicht, was im folgenden Kapitel untersucht wird. 
3. Die umgekehrte Perspektive im Paris-Kapitel der "Lalka"

Bei der Benützung des Begriffs Perspektive geht man automatisch von dem Fall aus, daB ein bestimnter Raum als Objekt vorliegt. Es handelt sich dabel aber um einen Sonderfall; denn, wie z.B. McLuhan nachweist, gehören wichtige Voraussetzungen dazu, den Raum von einem bestimmten bzw. bestimmbaren Ort aus als solchen zu betrachten. Es handelt sich dabel um eine küstlich geschaffene Situation. Will man nun diesen als Objekt vorliegenden Raum darstellen, und zwar für jemand, der sich nicht in diesem Raum befindet, so ist die sprachwissenschaftich relevante Frage, welche Mttel die Sprache zur Verfügung stellt, bzw. vom Darstellenden aus gesehen, welche von den für den Raum bereltstehenden Mitteln, also Zeichen, für dieses spezielle Objekt die angemessensten sind.

Eine andere Frage lautet, und sie ist vom literaturwissenschaftlichen Standpunkt aus relevant, was der Darstellende einem nicht anwesenden Sprachteilnehmer über einen Raum mittellen will. Denn bei Situationen dieser Art, die man in jedem Erzählwerk finden kann, findet man Ergebnisse, bei denen nach den obigen Gesichtspunkten nicht adäquate Zeichen ausgewählt würden, sondern betont Unwichtiges und Irrelevantes genannt würde. Auch hier ist. ein sprachlicher DarstellungsprozeB anzusetzen, sein Ergebnis steht jedoch nicht mit dem im Einklang, was man von einer Raumdarstellung erwartet. Da für den zuerst geforderten Fall in der Wissenschaft vom medialen Darstellen der Begriff der Perspektive gefordert wird, nenne ich Erscheinungen der zweiten Klasse die ungekehrte Perspektive. Die Effekte, die mit dieser Technik erreicht werden, sind sehr verschiedenartig und müssen für jeden Fall gesondert charakterisiert verden.

Welche räumlichen Gegebenheiten der Stadt Warschau Prus für den Schauplatzaufbau des Romans "Lalka" verwendet, ist in verschiedenen Arbeiten untersucht worden 1. Die Rekonstruktion dieses Schau-

1 Godlewski, S.: Gdzie był sklep Wokulskiego? in: Gazeta Warszawska Nr. 1, 1932; ders., Adres prywatny bohatera "Lalki", ebenda Nr. 7; ders., Grzeniewski, L.B. und Markiewicz, H.: Sladami Wokuiskiego. Przewodnik literacki po warszawskich realiach "Lalki", Warszawa 1957. 
platzes aus den Fakten, wie sie im Laufe des Romangeschehens auftauchen, kann zwar eine topographische Komponente des Werkes freilegen, nicht aber alle Kriterien der künstlerischen Raumfiktion. Dies tritt besonders zutage an den Stellen des Romans, wo sich das Geschehen vorübergehend auf einen anderen Ort verlagert, und zwar nach Paris. Hier kann von einem Schauplatzwechsel gesprochen werden. Welche strukturellen Funktionen des Schauplatzes können durch die Gegenüberstellung von Paris und Warschau freigelegt werden?

Die Situation ist folgende: Der Held Wokulski ist durch die Iiebe zu einem Mädchen, namens Isabella, aus den ruhigen und geordneten Bahnen des Lebens geschleudert worden. Von Zweifeln gepeinigt, folgt er der Einladung eines Geschäftsfreundes nach Paris und erlebt die Eindrücke dieser Stadt am ersten Tag mit aller Intensität. Das Auftauchen dieses Raumes im Roman findet durch die Perspektive der Figur statt, der Autor greift in keinem Falle auf seine eigenen Kenntnisse zurück.

Die ersten Eindrücke bei der Ankunft des Zuges sind Lärm, Geschrei, Gerenne 1 (S. 7), ferner ein "Platz mit einer Menge von Omnibussen, ein- und zweispännigen Wagen, mit Kutschern, die entweder vorne oder hinten untergebracht sind." (S. 7) Wie betäubt fährt er ins Hotel, "ein ungeheures, fünfstöckiges, keilförmiges Gebäude." (S. 8) In seinem Zimmer angelangt, legt er sich zunächst aufs Bett und erlebt folgendes: "Er war eingetaucht in zwei Geräusche. Hinter den Türen, auf dem Hotelkorridor, unterhielten sich die Leute und liefen, als ob gerade im Augenblick etwas passiert wäre; hinter dem Fenster, auf der Straße ertönte ein unbestimmter Lärm, welcher aus dem Gerassel verschiedener Wagen bestand, aus Glockentönen, Menschenstimmen, Hupen, Schüssen und weiß Gott was, aber alles gedämpft und entfernt." (S. 10) Hier kann man feststellen, daß die Geräusche vom Autor nicht in ihrer räumlichen Verteilung als Mittel zur Raumschaffung charakterisiert und differenziert werden, sondern so genannt werden, wie sie der Held empfindet, wie er versucht, Bekanntes herauszuhören und so in ihrer verwirrenden Vielfalt seinen eigenen Standpankt $z u$ behaupten. Gerade

1 B. Prus: Lalka, Warszawa 1949, Bd 1-2. 
Schüsse sind nicht ein typisches Geräusch für eine Stadt. Die nächsten Eindrücke empfängt er beim anschließenden Spaziergang durch die Straßen: "Eine breite Straße, mit Bäumen bepflanzt. In einem Augenblick fliegen an inm ungefähr sechs Wagen vorbel sowie ein gelber Omnibus, beladen mit Reisenden im Innern und auf dem Dach. Rechts, irgendwo weit weg, ist ein Platz zu sehen, links - hinter dem Hotel - eine kleine Markise ..." (s. 11) Der Held versucht, von den Eindrücken Abstand zu gewinnen, indem er zählt und sich in der Richtung orientiert: sechs Wagen, rechts und links. Die Gebäude werden nicht mit dem Ziel beschrieben, in der Situation des Lesers im Laufe des Leseaktes ein Feld aufzubauen, in welchem alle Wahrnehmungsdaten ihre Bedeutungspräzision finden und in ein Erlebnis der Stadt Paris einmünden. Präzisiert wird die Situation des Helden; er will aus seinem Wahrnehmungsumfeld eine Auswahl treffen und durch das Finden von Zeichen für diese Gegenstände die Komponenten seiner eigenen Situation klarstellen, damit sie durch diese Nennung ihre Unklarheit und Unsicherheit verliert. Durch diese Technik erreicht der Autor zwar keine anschauliche Darstellung der Stadt Paris, ihrer topographischen Anordnung und architektonischen Schönheiten, aber eine anschauliche Schilderung der Situation des Helden.

"In der Tiefe links ist irgendein gewaltiges Gebäude zu sehen. Im Parterre trägt es eine Reihe von Arkaden und Bildsäulen, im ersten Stock riesige Säulen aus Stein und etwas kleinere aus Marmor mit vergoldeten Kapitellen." (S. 12) Er vergleicht das Gesehene mit Warschau: "Er erinnert sich an sein Geschäftshaus, das Schmuckstück der Stadt, errötet und geht weiter." (S. 12) Die Wesensmerkmale der Ortlichkeiten und Gebäude sind dem Bewrutseinshorizont des Helden eingeordnet. Der Autor will nur indirekt einen Eindruck dieses Raumes beim Leser hervorrufen, in erster linie aber den Eindruck auf den Helden schildern. "Die Dächer sind irgenwie originell, beladen mit Kaminen, diese wiederum voller Kaminchen und Spitzen aus Blech. Und auf den Straßen wächst auf Schritt und Tritt entweder ein Baum empor oder eine Laterne, oder ein Kiosk, oder eine Säule, die mit einer Kugel aufhört." (S. 14) Immer weiter entfernt sich das hier Gebotene vom real Gegebenen; ein Leser, der Paris kennt, wird sich nicht orientieren und die 
jeweiligen Orte nicht identifizieren können. Dafür wird das Erleben des Helden in neue Bilder gekleidet: "Wokulski scholnt es, daB er, aus kaltem Wasser herausgezogen, plötzlich ins sledende Wasser gefallen wäre, welches braust und rauscht und spritzt." (S. 15) Präzisierung des figuralen Erlebens erfolgt nicht durch die Präzisierung des Raumaufbaus, sondern durch Verstärkung des subjektiven Moments. Der Wahrnehmungsraum als Umfeld der Figur wird vom Autor nicht weiter differenziert in der Form, wie er in einem bestimmten Augenblick vorliegt, sonderm ein wahrgenommener raumfüllender Gegenstand wird Ausgangspunkt für ein neues Feld. Dadurch stellt der Autor die Situationskomponenten des Helden zusammen und schildert sein Bestreben, seinen eigenen Standpunkt zu finden, wozu er ihn in einen neuen, unbekannten Raum führt. Die Masse der neuartigen Eindrücke will ihn erdrücken, Wokulski muß sich dagegen wehren, von diesem Raum aufgesaugt zu werden. Er erlebt nicht seine Bewegung durch die Straßen, sondern die Straßen und Bilder ziehen an ihm vorüber und erschöpfen sich schlieblich in ihrer überwältigenden Neuartigkeit. "Das Ende der Menschenmassen ist nicht $z u$ sehen ... Er hört auf, auf das lärmende Gespräch zu hören ... Dann wird er taub für das Geschrei der Straßenhändler ... Dann scheint es ihm so, als ob er schon einmal irgendwo solche Häuser gesehen hätte, solch eine Bewegung, diese Cafes; später denkt er, daß es schließlich nichts Großartiges ist, und endlich erwachen in ihm kritische Fähigkeiten ..." (S. 15)

Immer wieder versucht er, das Neuartige und tberwältigende in vor. handene Kategorien einzuordnen. Unter dem Aspekt der Frage nach dem Schauplatz im Roman ist dabei festzustellen, daß in diesem Falle seine Macht und GroBartigkeit 2 war genannt, aber nicht direkt in der Phantasie des Lesers hervorgerufen werden. Der Erzählvorgang ist nicht bestimmt durch die Illusion, als ob der Leser sich in diesem Raum befände und staunend umherblicke, so daß immer neue Bilder vor seinen Augen entstehen. Die Verändemungen in der Situation, im inneren Zustand des Helden werden durch die Technik der Schauplatzverlagerung dargestellt. Der Autor koordiniert die beiden Schauplätze, indem er auch für den neuen Schauplatz gewöhnliche Formulferungen, möglichst einfache und vertraute Kategorien zu finden sucht, in welche das Wahrge- 
nommene eingeordnet werden kann. Wenn es also heiBt: "Was ist denn das schon wieder ...., denkt er, als er zur linken ein ungeheures Gebäude sieht, unvergleichbar mit irgendeinem von denen, die er bis jetzt kennengelernt hat." ( $S .16$ ), so ist man auf einen ganz auBergewöhnlichen Gegenstand gefaßt. Es heiBt aber weiter: "Es ist ein KoloB, ein Rechteck aus Stein, darin ein Tor mit halbkreisförmiger Kuppel, offensichtlich ein Tor, welches an der Kreuzung von zwei Straßen steht ... Ein paar hundert Schritt weiter ein ähnliches Tor, und zwischen thnen eine breite Straße, die sich nach rechts und links hinzieht." (S. 16) Wenn man sich nur an diese Gegebenheiten hält, hat man ein Gebäude vor sich, welches ganz uninteressant ist und das sich vorzustellen sich nicht lohnt. Vom Arc de Triomphe erfährt man nur: "Auf beiden Seiten Plätze und Paläste, und weit weg, auf einer Anhöhe, ein riesiges Tor. Wokulski fülte, daß ihm auf diesem Platz die Eigenschaftswörter und die höchsten Grade fehlen." (S. 27) Dabei hat er auch schon vorher keine angemessenen Attribute gefunden. Wesentlich ist dem Autor, den Versuch des Helden, seinen Standpunkt im Raum zu behaupten, darzustellen und ihn möglichst unangemessene, der Pracht der Wahmehmungsgegenstände nicht gerecht werdende Formulierungen finden zu lassen.

Beim Versuch, diesen Abschnitt zu charakterisieren, kann man Anklänge an das Motiv des sagenhaften Ganges durch den Garten der Versuchung finden; dafür spricht auch das Erlebnis des Helden beim Blick in den Spiegel des Hotelzimmers: "Wokulski fühlte, daB er aus diesem Zimmer fliehen mußte. Das Gespenst, vor welchem er aus Warschau geflüchtet war, war auch schon hier und stand hinter der Schwelle." (S. 11) Diese Flucht ist das Thema dieses Paris-Kapitels, nicht die räumlichen Gegebenheiten der Stadt als ästhetischer Selbstzweck. "Er ging durch die Champs Elysées und murde betäubt durch die nicht endende Reihe von Kutschen und Wagen, zwischen welchen sich Reiter und Reiterinnen durchdrängten. Er ging und vertrieb die finstren Gedanken, welche wie eine Schar von Fledermäusen über ihm kreisten. Er ging und wagte nicht, sich umzuschauen; es schien ihm, $d a B$ auf diesem Weg, der vor Pracht und Freude sprudelte, er als einziger ein zertretener Wurm sei, der seine Eingeweide hinter sich herzieht." (S. 28) Dieses außer- 
gewöhnliche Bild sticht von allen übrigen $a b$; es ist kein eigentliches Bild, weist vielmehr auf den Gesamtzusammenhang des Romans hin und ist ein Zeichen dafür, daß das ganze Kapitel des Parisaufenthaltes seine Strukturrelevanz nur als eine Stufe in EntwicklungsprozeB des Helden erhält. Das Auftauchen von Bildern, die den stilistischen Horizont der Figur übersteigen, deren Elemente aber in ihrer Zusammensetzung von der Perspektive der Figur abhängig sind, ist bezeichnend für das Werk von Prus und dient als Technik, die Situation der Figuren zu präzisieren.

Der Schluß dieses ersten Tages in Paris und die damit verbundene Folge von Eindrücken ist auch durchaus nicht durch einen Höhepunkt oder durch die Vollständigkeit der möglichen Eindrücke gebildet, sondern durch das gleiche Bild, welches von allen Gebäuden gegeben wird, und den Umstand, daB der Held die Lust verliert, weiter seine Aufnahmefähigkeit einzusetzen. "Vom Platz aus bog er in irgendeine Straße ab, wo sich nach rechts ein Garten hinzog, aufgeteilt durch eiserne Staketen und Säulen, auf denen Blumen standen, rechts eine Reihe von Häusern mit halbrunden Dächern, mit einem Wald von Kaminen und Kaminchen, mit nicht endenwollenden Ballustraden ... Er ging langsam und dachte kummervoll, daß nach einem kaum achtstünigen Aufenthalt Paris ihn zu langweilen beginnt ..." (S. 28) Die strukturelle Einordnung dieses Raumes in den Gesamtzusammenhang erfolgt dadurch, daß einerseits die mit dem Erleben des Helden verbundenen Formulierungen beendet werden, der Autor Jedoch diesen Erlebnisvorgang über die Dauer des figuralen Wahrnehmungsaktes weiterführt. Die dynamische Bewegung der Bilder, die in ihrer Wahrnehmungsfolge abbricht, geht auch beim Schließen der Augen weiter und zeugt von einem Erleben räumlicher Art, welches von einer Auslösung durch reale Gegebenheiten unabhängig, aber deshalb genauso intensiv ist. Der so erlebte Raum erhält Strukturfunktionen, die nicht mehr mit dem Begriff des Schauplatzes charakterisert werden können. "Er löschte die Lampe. Auf der Straße war es still; durch das Fenster flob ein graues Leuchten der Lampen, welches wahrscheinlich von den Wolken reflektiert wurde. Aber Wokulski rauschte und klang es in den Ohren, und vor den Augen erschienen ihm die StraBen glatt wie ein Parkett, dann wieder Bäume, von eisernen Körbchen umfaßt, 
dann Gebäude, aus ungeheuren Steinen erbaut, dann wieder eine Schar von Menschen und Wagen, unbekannt, woher sie kommen, unbekannt, wohin sie fahren. Beim Betrachten dieser furchteinflöBenden Erscheinung schlief er ein und dachte, daß jedenfalls dieser erste Tag in Paris inm fürs ganze Leben in Erinnerung bleiben werde.

Dann träumte er, daß dieses Meer von Häusern, der Wald von Standbildern und die unendlichen Reihen der Bäume über ihm sich anhäufen, und $d a B$ er allein in diesem unermeBlichen Grabgewölbe schläft, ruhig, fast glücklich." (S. 29)

Der Umbruch in der psychischen Entwicklung des Helden wird nicht dadurch verdeutlicht und demonstriert, daß die Vertrautheit mit dem umgebenden Raum schwindet, sondern indem der Autor den Helden eine Fahrt in ihm unbekanntes, fremdes Gebiet unternehmen läßt. Denn die in Paris stattfindenden Gespräche und Handlungen stehen in keinem direkten Zusammenhang mit dem auf der Warschauer Bühne sich abspielenden dramatischen Geschehen und dienen nicht unmittelbar der Weiterentwicklung und Lösung der Konfliktsituation. Kontinuität besteht jedoch im Erleben des Helden, der zum Bewußtwerden des eigenen Standpunktes an einen fremden Ort geleitet wird und seiner Orientierungsmöglichkeiten verlustig geht.

Dieser neue Raum wird nur in den Romanverlauf miteingeschaltet, soweit es die Auseinandersetzung des Helden mit ihm erfordert. Die Beobachtungsperspektive ist in den Standpunkt des Helden verlagert; dessen Waffen sind sprachliche Formulierungen, die den Raum, der ihn aufsaugen will, als objekt behandeln, d.h. ihn auf technische Details und meBbare Größen beschränken. Er sieht nur das, wofür ihm eine Bezeichnung oder eine Parallele aus der Heimat zur Verfügung steht. Ebendies sieht auch der Leser, und in der Spannung zwischen dem Raum, der in seiner Gewalt tatsächlich vorhanden ist und empfunden wird, und dem Raum, der in den Formulierungsversuchen der Figur zum Ausdruck kommt, beruht der ästhetische Zweck und die strukturelle Bedeutung des Paris-Kapitels. Das Erlebnis des Helden ist kontinulerlich, es weist über das Ende des Kapitels hinaus, es ist mit dem Schließen der Augen 
nicht vorbei. Obwohl alles ruhif ist, rauscht os in Nokulskd. Ohren. Die Bewegung geht, gegenstandsunabhängif geworden, in der Phantasie des Helden weiter und schlägt um in einen Traum, in dem der eigene Standpunkt auf nevartige und verfestigte Neise erlebt wird: "ruhiB, last glücklich." (S. 29) 
4. Integration von Schauplatz und Handlung in Reymonts "Chłopi"

Alles, was im Roman "Chłopi", geschrieben 1904-1909, geschieht, spielt sich im Dorf Lipce und seiner Umgebung ab. Doch kommt dem Leser bei der Lektüre dieses monumentalen Werkes die Beschränkung auf einen kleinen Ort zum BemuBtsein? Wohl nur, wenn er sich nach den Ausmaßen und der Natur des Schauplatzes fragt. Doch wird dieser in Form von stets neuen und originellen Landschaftsbildern und Naturerlebnissen dargestellt, so daß seine Funktion nicht allein in der Bildung eines Feldes besteht, in welchem die Handlungen der Figuren stattfinden und die mit ihrer Darstellung verbundenen Zeichen ihre Bedeutung erfüllen. So kann man 2war den gesamten Erzählverlauf einteilen in solche Abschnitte, die der Fortführung der figuralen Handlung dienen, und solche, die den Ort beschreiben, wo dieses stattfindet. Doch löst der Autor die letzteren aus ihrer situationsbedingten Abhängigkeit vom figuralen Handlungsbereich heraus, indem er der Natur eigenständiges Leben und immanente Vorgänge zukommen läßt.

Der Schauplatz wird durch ein Bild eingeführt, dessen Komponenten von einem Standpunkt zeitlich unabhängiger Natur und ohne Bindung an eine Situation des Handlungsverlaufes gesehen sind. "Stille lag auf den leeren Feldern und eine berauschende süße war in der vom Sonnendunst gedämpften Luft; im hohen, blassen Blau lagen hie und da unordentlich hingestreute, riesige weiBe Wolken, wie von den Sturmwinden angewehte und zerrissene Schneewälle.

Und unter ihnen, soweit das Auge reichte, lagen graue Felder wie eine ungeheure Schale mit eingekerbtem Rand aus dunkelblauen Wäldern, eine Schale, durch welche wie ein in der Sonne silbern aufblitzendes Gespinst ein FluB in Windungen zwischen Erlen und Uferweiden hervorflimmerte. Er staute sich mitten im Dorf zu einem riesigen länglichen Weiher und verlief weiter nach Norden durch eine Schlucht zwischen den Hügeln. Auf dem Grund der Mulde, rings um den Weiher, lag das Dorf und schillerte in der Sonne und den Farben der Gärten - wie eine rotgelbe Raupe auf einem grauen Lattichblatt ..." 1

1 w. St. Reymont: ChYopi, Warszawa 1952, Bd 1, s. 7. 
Der Standpunkt des Autors befindet sich in einer Position, die inm einen weiten Blick in diese Landschaft erlaubt. Er sieht Felder, den blauen Himmel mit weiBen Wolken, dunkelblaue Wälder, einen FluB, ein Dorf mit einem Weiher, dahinter Hügel und eine Schlucht. Neben diesen Gegebenheiten trägt das Bild noch ganz andere Komponenten. Sie sind von den räumlichen Gegebenheiten unabhängig, am Aufbau des Bildes aber wesensmäBig viel stärker beteiligt als diese Ortlichkeiten. Sie sind die für das Bild eigentlich verantwortlichen Faktoren. Diese andere Klasse von Elementen, die ebenfalls Naturgegebenheiten sind, gelangt auf dem Wege des Vergleichs in das Bild: Die Wolken sind wie Schneewälle, die Felder wie eine Schale, der FluB wie ein Gespinst, das Dorf wie eine Raupe auf einem Lattichblatt. Dabei fällt auf, daß diese Vergleichsmomente durchwegs Gegenstände sind, die sehr viel kleineren Kategorien entstammen.

Dieses unmittelbare Nebeneinander von extrem großen und extrem kleinen Gegebenheiten kann noch weiter verfolgt werden. Die Schale und der Weiher sind ungeheuer bzw. riesig, zwischen den Hügeln befindet sich eine Schlucht. Die Hervorhebung dieser Kontraste oder gar Gegensätzlichkeiten ist ein Ergebnis der Analyse, das beim eigentlichen Leseakt nicht ins Bewußtsein tritt. Die Schaffung des Bildes beruht nicht auf einem künstlichen Arrangement der vorgefundenen Raumteile, sondern ihre sprachliche Verarbeitung in Form des Vergleichs schafft erst die Komponenten, die sich zu einem Bild zusamenschlieBen, so daB das Bild nicht so sehr in räumlichen Perspektiven vor dem Auge des Lesers steht, sondern in Form von Figuren, die sich zu einem Bild vereinigen.

Daß diese literarischen Figuren erlebt werden und den ästhetischen Effekt ausmachen, wird besonders deutlich bei der Untersuchung des nächsten Bildes unter besonderer Berücksichtigung der akustischen Phänomene.

"Reihen von Frauen leuchteten auf den Kartoffeläckern rot auf ... das Kollem beim Ausleeren der Kartoffeln wurde hörbar ... an manchen Stellen wurde noch für die saat gepflügt ... Herden buntscheckiger Kühe weideten auf den Brachfeldern ... lange, aschfarbene Saatfelder begannen sich mit dem sprieBenden Getreide rostrot $z u$ färben ... Wie Schneeflocken schimmerten weiße Gänse 
auf den dürren, verschossenen Wiesen ... irgendwo muhte eine Kuh ... die Feldfeuer brannten und lange blaue Rauchstreifen zogen sich über den Ackern hin ... ein Wagen rasselte oder ein Pflug knirschte gegen einen Stein ... dann umfaBte wieder Stille für einen Augenblick die Erde, daß man das gedämpfte Plätschern des Flusses und das Klappern der hinter dem Dorf im Dikkicht der zusammengedrängten gelben Bäume verborgenen Mühle hören konnte, dann flog wieder ein Liedchen auf oder ein Schrei flog, unbekannt woher, niedrig dahin, flatterte tief über die Furchen und Rillen und versank ohne Echo im herbstlichen Grau, in den Stoppelfeldern, die von silbernen Spinnweben überzogen waren, in den leeren, verschlafenen Wegen, über welchen die Ebereschen ihre blutroten, schweren Köpfe neigten ..." (S. 9) Auch bei diesem Bild liegt die Vogelperspektive vor; man sieht nämlich mehrere Kartoffeläcker, Saat- und Brachfelder, einige dürre Wiesen, den Fluß, das Dorf und einige Wege. Im Gegensatz zum obigen Bild liegt dabei die Betonung gerade auf der Unklarheit, Unbestimmthe1t und Ungenauigkeit der Lokalisation. Aufschluß über die Technik, wie dieses Bild entsteht, ergeben die Laute, und zwar sind es folgende: Kollern, Muhen, Rasseln, Knirschen, Plätschern, Klappern, ein Liedchen, ein Schrei. Es sind durchwegs Wörter, die davon Zeugnis ablegen, daß ihr Benutzer auf die spezielle Eigenart des Geräusches besonderen Wert legt, lautmalende Verben, die mit ihrem phonetischen Bestand die Nachahmung anstreben, und das heiBt, die von der Situation und den Gegebenheiten, von denen sie ursächlich abhängig sind, freigemacht sind. Die beiden letzten Erscheinungen, das lied und der Schrei, sind zwar nicht lautmalender Natur, erfahren aber eine literarische Sonderbehandlung: Sie fliegen in einer visuell genau nachprüfbaren Richtung und verschwinden an genau fixierbaren Orten.

War schon beim ersten Bild das Ergebnis der Analyse, daß die Wirkung der sprachlichen Gestalt bedeutungsmäßig vor der realen und nachprüfbaren räumlichen Situation steht und für den Charakter des Bildes in erster Iinie verantwortlich ist, so erhält man an dieser Stelle ein neues Argument. Die Natur der hier angeführten Laute, ihre rein akustische Seite, steht im Widerspruch zum visu- 
ell Wahrgenommenen und dessen Dimensionen, wenn man die Vogelperspektive in rein räumlichem Sinne auffaßt. Von einer Stelle aus nämlich, von der man einen so umfassenden und weiten Blick in die Landschaft tun kann, ist es nicht möglich, Geräusche und Laute von subtilem Charakter wie Steineknirschen und Wasserplätschern überhaupt zu hören. Die strukturelle Funktion dieser Erscheinungen liegt also wiederum in ihrer Wirkung literarischer Art. Die Integration liegt im ZusammenschluB zu solchen Figuren und nicht in der spezifischen Anordnung zu einem einheitlichen Raum.

Eine ähnliche Irrelevanz der räumlichen Lage wahrgenommener Gegenstände tritt auch dort auf, wo das Dorf als Darstellungsobjekt den Erzählverlauf bestimmt. Auch hier tauchen verschiedene Techniken auf; das eine Mal ist das Dorf ein semantischer Oberbegriff, unter welchem eine Reihe von verschiedenen Erscheinungen und Eindrücken zusammengefaßt wird, wobei die einzelnen Bildelemente nicht notwendigerweise räumlicher Natur sind, d.h. ihre Position im Raum nicht in Form eines fixierbaren Standpunktes der Wahrnehmung berücksichtigt wird. Auch hier tritt wieder die Diskrepanz von visueller und akustischer Perspektive zutage. Der Autor sieht einerseits die Häuser in einer perspektivischen Verkleinerung, also aus groBer Entfernung, andererseits hört er Geräusche, wie sie nur in unmittelbarer Nähe wahrnehmbar sind. "Das Dorf versank in die Dunkelheit und in die tiefe, tote Stille des Herbstabends. Die Bauernhäuser wurden kleiner, als duckten sie sich zur Erde, als schmiegten sie sich an die sohläfrig geneigten Bäume, an die grauen Hecken." (S. 24) "Im Dorf war es schon dunkel, durch die angelehnten Türen und Fenster strömten in den warmen Abend der Schein des Feuers und der Geruch gekochter Kartoffeln und saurer kiehlsuppen mit Speckgrieben. Hier und da aB man auf den Dielen oder auch vor den Häusern, daß man das Kratzen der Löffel hören konnte und die Unterhaltungen." (S. 26)

Auf der anderen Seite steht die Unterordnung der Elemente unter eine Gesamtstimmung, unter die charakteristische Wirkung einer bestimmten Tages- bzw. Jahreszeit, unter einen einheitlichen, thematisch geschlossenen Vorgang, im folgenden Beispiel etwa nit 
der tberschrift: Aufbruchsstimmung am Morgen. "Im Dorf begann schon das alltägliche Treiben: Der Morgen war klar und kalt, und da der Frühreif die Luft erfrischt hatte, bewegte man sich munterer und geräuschvoller; die einen gingen in Gruppen auf die Felder zum Kartoffelausnehmen mit Hacken und Körben in der Hand und kauten noch das Frühstück; andere zogen mit dem Pflug auf die Stoppelfelder; andere hatten die Eggen auf dem Leiterwagen und Säcke voller Saatgetreide; andere schlugen den Weg nach den Wäldem ein mit Harken auf den Schultern, um Nadelstreu zu holen - so rasselte es auf beiden Seiten des Teiches und das Geschrei wurde stärker, denn in dem Wege drängten sich Vieh, das auf die Weide zog, das Hundegebell, die Zurufe, die immer wieder aus dem niedrigen, schweren Staub, der sich von den taufeuchten Wegen erhob, erschollen." (S. 43) Jean Paul d'Ardeschah übersetzt den letzten Satz folgendermaßen: "... denn auf den Wegen drängte sich das Vieh, das auf die Weide zog. Hundegebell und Zurufe drangen aus den niedrigen, schweren Staubwolken, die sich von den taufeuchtenWegen erhoben." 1 Im Original gibt es jedoch nur ein Verbum für Vieh, Hundegebell und Zurufe, die Lokalisation ist jedoch zweimal mit den "Wegen" angegeben.

Die Zurufe werden also auf folgende Weise beschrieben: "... in den Wegen drängten sich ... die Zurufe, die aus dem .... Staub, der sich von den ... Wegen erhob, erschollen! Auf diese Weise reduziert, gibt der Text unschwer zu erkennen, daß nicht die räumliche Präzisierung Intention des Autors ist. Die Lokalisation ist hier zweimal mit den "Wegen" angegeben, wodurch ein präzis orientativer Nachvollzug unmöglich wird, demalle Vorgänge spielen sich auf diesen "Wegen" ab. Am Ende dieser langen Satzperiode tauchen sie nochmals auf, und hier dienen sie als Träger des Attributes "taufeucht", wodurch das Bild um eine zusätzliche Komponente bereichert wird. Die im informativen Sinne redundanten Wörter fungieren also als Glieder, um die eine Reihe von ornamentalen Attributen gruppiert sind.

Diese Technik ist bei allen Schilderungen von Ortlichkeiten festzustellen: Die Angabe räumlicher Positionen dient nicht der Ori-

1 W. St. Reymont: Die Bauern, Düsseldorf-Köln 1956, S. 41. 
entierung des Lesers auf einem Schauplatz, sondern sie dient als Träger für weitere Attribute, welche den lebendigen Effekt dieser Bilder verstärken, ja ihn eigentlich ausmachen. Diesen Effekt bezeichne ich mit räumlicher Dynamik, obwohl das künstlerische Gestalten sich nicht auf die erwarteten oder nachprüfbaren Elemente des realen Raumes erstreckt. Doch gilt auch hier der Grundsatz, $d a B$ jede Vorstellung eine räumliche Komponente enthält, und bei den in diesem Werk auftauchenden Bildern ist die Vermittlung eines Erlebnisses der Bewegung sicher sehr groB. Dazu noch ein Beispiel einer Waldszene, in der auf seiten der räumlichen Position der Dinge keine Bewegung vorliegt, sondern äuBerste Ruhe: "Es war ein alter, mächtiger Forst. Eichen, Fichten und Birken drängten sich in einer dichten Schar zusammen, und unter ihnen schmiegte sich an die dicken Stämme das kleine Volk der Haselbüsche, Espen, der Wacholdersträuche und Hainbuchen; an manchen Stellen breiteten sich Tannenschonungen trotzig aus und streckten sich gierig nach dem Sonnenlicht.

Auf dem Weg glänzten noch überall die Pfützen vom gestrigen Gewitter, und abgebrochene Aste und Baumkronen lagen umher, und hier und da lag sogar ein kleines Bäumchen, das mitsamt den Wurzeln ausgerissen worden war, wie eine Leiche quer über dem Weg. Es war ganz still, kühl und dämmerig, es roch nach Moder und Pilzen, die Bäume standen bewegungslos wie im Anblick des Himmels versunken, und hier und da drang die Sonne durch das dichte Laub. Die Lichtstrahlen krochen wie goldene Spinnen über das Moos, über die roten Beeren, die wie geronnene Blutstropfen aus dem bleichen Gras hervorkrochen." (Bd. 4, S. 212)

Der Aufbau dieses Bildes erfolgt nach dem Prinzip der belebten Natur. Man braucht nicht auf jedes einzelne dieser Elemente hinzuweisen, um zu zeigen, daß alle Bewegungen, die auch unbewegten Gegenständen durch ihre sprachliche Gestaltung zukommen, dem Bereich menschlicher Emotionen entspringen. Aus diesem Grunde bezeichne ich diese Bewegung mit "naturimmanentes Leben". Es kommt zustande, indem selbst unbewegten Elementen eine auf uneigentlicher Redeweise basierende Komponente der Bewegung zugeordnet wird. Fragt man nach der Integration dieser Bilder in das Romanganze, 
so muß man das Verhältnis dieses naturimmanenten Lebens zum Leben der Figuren, d.h. zum dramatischen Handlungsablauf analysieren. Das oben zitierte Beispiel ist folgendermaßen eingeordnet. Antek, der gerade eine heftige Auseinandersetzung auf einer Versammlung von Bauern mitgemacht hat, gerät auf dem Heimweg durch eben diesen Forst. Er ist von der ihn umgebenden Szenerie unberührt; seine Gedanken sind bei dem vorausgegangenen Streit mit den Beamten, wenig später setzt er sich unter einen Baum und schläft ein. Auf diese Weise lösen sich den ganzen Roman hindurch dramatisch geschlossene Eimeiten ab, einerseits auf die Figuren bezogen, andererseits auf den Raum, und das bedeutet hier ausschließlich: auf die Natur. Eine Korrespondenz ist nur auf dem Sektor der Größenordnung erlebnismäßiger Kategorien zu finden. Das bedeutet, daß sich tberschneidungspunkte und Abhängigkeiten der beiden Bewegungsarten, der beiden LebensäuBerungen da einstellen, wo Höhepunkte in einer dramatischen Folge vorliegen; also bei liebe, Haß, Tod, Gefahr usw., wo das Romangeschehen sich in schicksalhaft bedeutsamer Weise zuspitzt, z.B. wie im Todesmoment des Helden Boryna: "Er blieb erstaunt stehen, es schien ihm, als bewegte sich ihm alles entgegen, die Gräser krochen, die wogenden Getreidefelder fluteten heran, die Ackerbeete umzingelten ihn, die ganze Welt erhob sich und kam auf ihn zu, die Angst packte ihn, er wollte schreien, konnte aber keinen Laut aus der zugeschnürten Kehle hervorbringen, er wollte fliehen, aber es fehlten die Kräfte und die Erde griff nach seinen Füßen, die Getreidehalme umspannten ihn, die Ackerfurchen hielten ihn zurück, die harten Schollen faBten ihn, die Bäume stellten sich ihm drohend in den Weg, die Disteln krallten nach ihm, die Steine verwundeten seine Füße, es jagte ihn ein böser Wind, die Nacht verwirrte ihn und die Stimmen, die durch die ganze Welt hallten: Bleibt! Bleibt!" (Bd. 3, S. 351) Hier bewegen sich nicht nur die Elemente der Landschaft, denen von Natur aus Beweglichkeit und Dynamik zukommt, sondern auch statische Gegebenheiten ergreifen die Aktivität. Der Raum ist nicht Ort der Handlung, sondern jedes einzelne seiner Elemente nimmt an der Handlung teil. Aber nicht der Raum als solcher wird von einer einheitlichen Bewegung ergriffen, sondern jedem Ding im Raum wird eine wirkungsmäßig 
5. Der symbolische Raum in Żeromskis "Przedwiośnie"

Bei der Untersuchung literarischer Räume und ihrer strukturrelevanten Funktionen sieht man sich immer wieder mit der Tatsache konfrontiert, daB zur Beschreibung dieser Funktionen Begriffe nötig sind, die über die spezifisch raumbezogene Terminologie hinausgehen. Man kann zwar immer nach der Orientierungsmöglichkeit, der Perspektive, der Nachprüfbarkeit und Effektivität der Positionsangaben fragen, wird aber an den künstlerischen Kriterien dieser speziell sprachlichen bzw. literarischen Raumdarstellung vorbeigehen.

Dies ist auch in Stefan Żeromskis letztem Roman "Przedwiośnie", geschrieben 1925, der Fall. Hier liegt ein historischer Raum vor, der zur Erhellung eines bestimmten Sinnes, einer tieferen Bedeutung dient. Er ist deshalb auch nie alleiniger Gegenstand einer Erzählphase oder eines Abschnittes. Vielmehr ist hier der Raum selber ein Medium, an dem ein gedanklicher Zusammenhang demonstriert wird.

Von den Beispielen solcher raumbezogener Erzählabschnitte soll zuerst die Beschreibung des Parks von Nawloc angeführt werden. Der Romanvorgang stellt die Entwicklung des Helden Cezary zu einer repräsentativen Figur im historisch-sozialen ProzeB dar. Eine Phase dieser Entwicklung vollzieht sich in Nawloc, einem alten Gut; dieses wird mit seinen Wohnräumen und Wirtschaftsgebäuden sowie den umliegenden Gebieten zum Schauplatz des Zusammenstoßes von althergebrachter Ordnung und den Ideen der sozialen Neuerung. Der Park ist nun nicht der Ort, wo dieser Konflikt ausgetragen wird, sondern seine Beschreibung dient der Veranschaulichung und Verdeutlichung der Natur dieses Schauplatzes, der ja nicht als eine einheitliche Bühne vorliegt, sondern durch häufigen Szenenwechsel bestimmt ist.

"Der Yark war sehr ausgedehnt, er reichte vom Hügel, auf dem die "Arianka" stand, bis ins Tal, bis zum Hof, der von kleinen Teichen und Wasserbassins umgeben war. Der Hof war aus Holz, aber auf eine steinerne Untermauerung gestützt, die einst einem anderen, höheren Gebäude als Fundament gedient haben mußte. Im 
Park waren lange Alleen von Weißbuchen, die sich in den Feldern erhoben, und weites Buschwerk. In einer dieser Alleen standen im Kreise morsche Holzbänke, mit welken Blättern bestreut und feucht vom Regenwasser. Alle Alleen und Wege waren von einem feuchten Dunst überzogen, der auf Cezary einen ganz bestimmten Reiz ausübte. Mit Genuß wanderte er zwischen den Weißbuchen, die wie ein Kirchenschiff beieinanderstanden, umher, ohne einer Menschenseele zu begegnen. Er umhüllte sich mit seinem luftigen Mantel und erlebte die Wärme in der Novemberluft, freute sich, berauschte sich zufrieden an seiner physischen Gesundheit und seinem Gefühl seelischer Behaglichkeit. Er sang halblaut ein Liedchen vor sich hin, welches skandalös dumm war, was den Inhalt betraf, und anspruchslos hinsichtlich seiner Form." 1

Diesen Abschnitt kann man in zwei Teile einteilen. Im ersten beobachtet der Autor die räumlichen Gegebenheiten, im zweiten die Gefühlserlebnisse des Helden. Der "feuchte Dunst" leitet zwar über in dessen Erlebnissphäre, jedoch an welchen Elementen des Raumes er seine Freude hat, wird nicht ausgefürt. Es sind Dinge, die einen interessierten Beobachter angehen, die Größe und Bauweise des Hofes, seine Geschichte und die Anlage des Parkes, aber obne Berücksichtigung erlebnismäßiger Momente. Mit diesen Zeichen wird ihr Erlebnis genannt, und auch die Art, wie dieses Ergötzen differenziert wird, läBt einen schulmeisterlichen Tonfall erkennen. Da ist die Rede vom gesunden Körper und von der gesunden Seele, von Form und Inhalt des Liedes. Die Wirkung des Raumes wird in der Form beschrieben, wie sie auf die Figur ausgeübt wird, aber sie wird nicht dem Leser vermittelt. Die Elemente des Raumes werden mit Zeichen versehen, der Abschnitt als Erzähleinheit entsteht erst durch ihre Kombination mit anderen Zeichen, welche die Erlebnissituation der Figur charakterisieren.

So wird die Landschaft, obwohl der Autor sie immer wieder beschreibt, nie anschaulicher und ästhetischer Selbstzweck. In der Erzähltechnik wirkt sich das so aus, daB der Blick des Autors erst dann auf die Landschaft fällt, wenn die Handlung in eine

1 Stefan Żeromski: Utwory wybrane Bd 5, Warszawa 1961, S. 98. 
statische, dramatisch relevante Phase gelangt, und nicht da, wo sie von der Figur zum erstenmal gesehen wird. "Cezary saß, den Rücken dem Pferd zugekehrt, und betrachtete zum erstenmal die Landschaft, die sich überraschend weit vor ihm ausbreitete. Die Felder zerfielen vor seinen Augen in zwei Teile, die sich nicht zu einer einheitlichen Ansicht verbinden wollten ..." (S. 105) Der Held befindet sich aber schon geraume Zeit in dieser Gegend. Als er das erstemal mit dem Reisewagen hindurchfuhr, hieß es jedoch nur: "Der Weg war wie geschaffen dafür, mit einem leichten herrschaftlichen Wagen und vier Füchsen schnell in die Ferne zu fahren." (S. 86)

Weitere Beispiele für diese unbildliche Raumdarstellung sind nicht nötig. Solche Abschnitte sind nie ausschließlich auf räumliche Gegebenheiten und Situationskomponenten beschränkt, da die Erzeugung einer Raumillusion nicht angestrebt wird. Vom Kriegsschauplatz führt der Autor zwar die nötigen Requisiten an, wie z.B. das Kriegsgerät, die rauchenden Brandstätten, die Ruinen; aber das Ende dieses Erzählabsatzes bildet die Beschreibung eines französischen Offiziers, der aus dem Haus tritt und mit einem Notorrad davonfährt. Durch dieses Detail, welches die räumliche Geschlossenheit des Bildes durchbricht, wird der szenische Charakter dieses Raumes hervorgehoben.

Die Verquickung von raumbezogenem Sprechakt und figuralem Wahrnehmungsvorgang, wie sie in der Erzählsituation gegeben ist, wird vom Autor formal geschieden und sprachlich getrennt realisiert. Die strukturelle Bedeutung des Raumes kommt also darin zum Ausdruck, daß ein Zusammenschluß räumlicher Bedeutungseinheiten zu einer geschlossenen Erzähleinheit immer dann erfolgt, wenn die Entwicklung des Helden in eine entscheidende Phase gelangt. Das ist in diesem Roman an den Stellen der Fall, an welchen das Leitmotiv des Vorfrülings auftaucht, nïmlich am Anfang, in der Mitte und am Schluß.

Jeder dieser Abschnitte bildet eine Struktur für sich. Die Gegenüberstellung der sich verändernden und der gleichbleibenden Situationskomponenten ist die Grundidee des Werkes. Die Strukturanalyse muß also die Gemeinsamkeit und die Unterschiede dieser 
drei Erzählabschnitte feststellen und fragen: Liegt hier eine Wiederholung von räumlichen Situationen bedeutungsvoller Art vor, oder handelt es sich um die Folge von verschiedenen Räumen, die einen EntwicklungsprozeB erkennen lassen?

Die erste Vorfrühlingsszene findet am Schwarzen Keer statt, wo der Held seine Jugendzeit verlebt: "Lange Stunden verbrachte er damit, das Meer zu betrachten, welches immer gleich und doch immer anders ist, zu welchem die Schichtungen der nackten Berge in jähem Sturz abfallen, über dem die zerzausten, abgestürzten Kiefern des Südens hängen. Als die Gräser die abschüssigen Gärten der schönen Bucht einhüllten, so daB sie in einem üppigen und leuchtenden Farbton grïn munden und dazwischen hundertfältig die Veilchen und Anemonen ihre lebendigen Augen öffneten und ihre kleinen Gesichter zeigten, erinnerte er sich an die Werke der Dichter, die man ihm in verschledenen Sprachen zu lermen befohlen hatte und die er früher gehabt hatte. Wenn sie ihren Duft verbreiteten, der immer das Gemüt bewegt, kam er zur tberzeugung, daß es nur zwei Dinge auf der Welt gibt, die nicht der Verderbnis und dem Tod anheimfallen: die ewige Wiederkehr der Blumen im Frühling und die Darstellung ihrer Wioderkehr auf die Erde in den Versen der Dichter. Er sagte jetzt der toten Mutter diese stillen, wie Veilchen durftenden Worte auf, die für niemand nützlichen, heimischen und fremden, die er früher nicht verstanden und nicht richtig eingeschätzt hatte. Er sandte ihr in die kalte Tiefe des Grabes durch diese Worte die Nachricht, daB der Früling aufs neue gekommen war, daß der kleine Vogel mit den goldenen schimmernden Federn, der Pirol, wieder da war. In glücklichen, vergangenen Tagen hatten sie sich gegenseitig auf ihn aufmerksam gemacht und seinem Gesang so gern in den Morgenstunden des Frühlings gelauscht. Er hatte sich von irgendwoher aufs neue gezeigt und in den Zweigen der einsamen Zeder mit seiner unverständlichen Sprache dem Himmel, dem Meer und der Erde das Glück der Wiederkehr des Vorfrühlings verkündet." (S. 33 f.)

Die Raumillusion ist sehr stark vom Stil beeinträchtigt; ein Zuviel an Fakten arbeitet einer anschaulichen Realisation des Gebotenen entgegen. Hervorstechend ist der Reichtum an Adverbien, nicht lokal präzisierender Art, sondern den Grad der Gefühls- 
intensität der Figur ausdrückend. Die glitzernden Farben haben nicht abbildende und veranschaulichende Funktion, sondern sie sind Träger eines Sinns. Die Schönheit wird zwar mit Worten zum Ausd mack gebracht, aber nicht so differenziert, daß sie in einem Erlebnis des Lesers resultieren könnte. Die Geruchsempfindung wird nicht illusionistisch übertragen, sonderm genannt und in den ProzeB der historischen Reflexion eingebaut: so duftet es immer. Der Blick auf den Raum ist Anstoß zu einem Sprechakt mit stilistischem Eigenleben, der sich im Romanverlauf als selbständiges sprachliches Gebilde konstituiert. Im Erzählvorgang dominiert der rhetorische Schwung über das Raumdetail. Das "kalte Grab der Mutter" schafft keine illusionsbedingte Vertikalachse, sondern wirkt metaphorisch im Sinne der Verdeutlichung des Sachverhaltes, daß nämlich das neue Leben den Tod überwindet. Dadurch entsteht eine Zeitachse: die Kontinuität des Lebens über den zeitlichen Rahmen und über den Ausschnittscharakter des Romans hinaus. Der Vorfrühling wird somit zu einer schicksalhaft bedeutsamen Situation. Im Roman wird er realisiert im Zusamenfallen der Situation des Helden mit einem historischen Augenblick der Menschheitsgeschichte, dem Vorabend der sozialen Revolution. Die tatsächlich zitierten Werke der Dichter werden nicht in den Erzählverlauf eingeflochten, sondern nur erwähnt und interpretiert. Den zweiten Vorfrühling erlebt der Held in dem Augenblick, da er das erstemal polnischen Boden betritt: "Es floB dort ein Bach, der sich in steilen Ufern durch die Niederung wand. Der Schnee war schon getaut und die ersten jungen Pflanzen begannen sich blaßgrün über das schnelle Wasser hin auszubreiten. Auf dem kaum sichtbar grünenden Wintergetreide tanzten barfuß ärmliche Knaben und spielten auf der Uundharmonika. Ihre nackten Sohlen, die schon imstande gewesen wären, auf der Tanzfläche in der Tenne zu stampfen, leuchteten im Schlamm. Der Vorfrühling hatte schon von den nächstliegenden Buden das Eis und den Schnee heruntergeblasen, sein südliches wehen erwärmte inr Inneres, welches der lange und schwere Winter, der Feind der armen Leute, mit seinem todbringenden Hauch umfaßt hatte. Abgerissene Regenrinnen, durchlöcherte Dächer, verschimmelte Wände bedeckte dieser unsterbliche Künstler, der herannahende Frühling, mit goldenen und silber- 
nen, grïnen und verschossenen Farbtönen, die er auf die Welt verteilte. Er strengte sich an, mit seinen unscheinbaren Farben diesen abstoßenden Anblick zu verbergen, welchen die Kenschen auf diesem Hintergrund der ewigen, unsterblichen Schönheit boten. Cezary schaute mit trübsinnigem Blick auf die morastigen Straßen voller grundloser Lachen, auf die Häuser von verschiedener GröBe, Form und Farbe und auf die AusmaBe, welche die Sudelei im Innern angenommen hatte, auf die Schweineställe und die Prützen, auf die Gebäude und die verbrannten Schutthaufen. Er kehrte auf den Marktplatz zurück, der von jüdischen Kramläden umgeben war, deren Türen und Fenster schon seit lionaten mit Dreck bespritzt, aber auch vorher schon ewig nicht mehr geputzt worden waren." (S. 76)

Die Motivsituation wird also wiederholt, Jedoch ibre Komponenten auf sehr abweichende Weise realisiert. Das erste Frühlingserlebnis am Meer ist beschaulich und idyllisch. Der Schauplatz der zweiten Realisation ist ein heruntergekommenes Städtchen. Die Situation beruht auf dem Kontrast des neuen Aufblühens und des halbverfallenen Bauwerks, wobei jedoch diese negative Komponente überwiegt. Die Wahrnehmungsgegenstände der Figur bilden das Material, aus welchem der Autor die Motivsituation aufbaut. Diese Fiktion erfolgt nicht in Form eines Sprechaktes der Figur, sondern als ein Sprachwerk des Autors, wofür die schwierigen und langatmigen Satzkonstruktionen sprechen. Die Situation der Figur wird transparent und läBt den allgemeinen und menschlich bedeutsamen Grundsatz erkennen, nämlich den unaufhaltsamen sozialen Abstieg an den Orten, wo ihm nicht aktiv Widerstand geleistet wird. Die Erzähleinheit entsteht durch die Verquickung von figuralem Erleben und auktoralem Interpretieren, durch die Konzentration von Raumdetails, die den Kontrast von Werden und Vergehen verdeutlichen.

Beim Erlebnis des dritten Vorfrühlings, mit welchem der Roman sein Ende nimmt, ist der Held bereits zu einer zentralen Gestalt des revglutionären Geschehens geworden. "Es war der erste Tag des Vorfrülings. Es wehte der Südwind und verwandelte die entlang der Gehsteige angehäuften Schneeberge in Ilüssigen Schlamm. In 
die. Haare, die $\mathbf{u}_{\ddot{u}}$ der, die Nasen, die Wangen und Obren der Kinder, die zur Schule eilten, blies jener trockene, welchselnde Wind. Tausende von Spatzen schwirrten fröhlich, äuBerst aufsässig, aber unerreichbar auf den Gesimsen der zerkratzten Mauern, auf den zerbrochenen Rinnen und den schwarzen Zweigen der Kastanie, die gefangen war im Hinterhof eines jüdischen Hauses. Blaßgrüne Jüdinnen steckten neugierig ihre bläulichen Nasen aus den Kellern und ihren Wohnungen in der Franziskanerstraße. In den sauberen Alleen gingen frähliche Passanten und stapften mit trokkenen Stiefeln auf dem Beton des Gehsteiges. Schon rührte sich an der Ecke ein junges StraBenmädchen in einem verfrühten Frühlingskleid, um mehr Nacktheit zum Anlocken der Männer zu zeigen. Eine Frühlingswolke 208 über der Stadt vorbei vie ein Engel Gottes, erfüllt mit gleicher Liebe für Tugendsame und Sünder. Eine Glocke ertönte von einem fernen Turm, als hätte ihr der Engel, der über den Himmel hinflog, aufgetragen, allen Elenden, Geplagten und von Krankheit Zerfressenen zuzurufen: Früling, oh ihr armen Erdenkinder!" (S. 240)

So zeichnen sich auf dem Hintergrund der Vorf rühlingsszenerie mit kontinuierlichem Phasenablauf und ewiger Wiederkehr drei Stufen in der Entwicklung des Helden ab: Zuerst die kindlich unschuldige Phase, voller Vertrauen auf die Kraft der Natur und des Lebens, dann die Enttäuschung über die zerfallenen Häuser, statt derer er auf Grund des väterlichen Yythos die utopischen "Glashäuser" (S. $14 \mathrm{ff}$. ) erwartet hatte; und schlieblich nach Uberwindung aller Rückschläge der optimistische Ausblick in eine bessere $\mathrm{Zu}-$ kunft. Gerade im letzten Abschnitt sind es nicht die einzelnen Komponenten des Schauplatzes, die auf ein Herannahen des Frühlings hinweisen, sondern das Zusammentreffen verschiedener Vorgänge. Eines dieser Details, das Vorüberziehen einer Wolke, wird durch den Vergleich mit einem Engel Sinnbild für die Idee, der Vorfrühling ist die frohe Botschaft für alle Armen.

Die Kategorie des räumlichen Erlebens rückt in den Hintergrund vor der historischen Transparenz der sinnlich gegebenen Dingwelt. Raumbezogene Erzählabschnitte sind durch stilistisches Eigenleben we lange Sätze, archaischen und überreichen Wortschatz charakte- 
risiert. Die anschauliche Wirkung des Raumes dauert gerade oder nicht einmal so lange wie die ihm gewidmete Erzähleinheit. Das bildhaft räumliche Zusammenspiel der Komponenten wird genannt, ohne den epischen Vorgang gestaltend zu beeinflussen.

Hier stellt sich neben die Frage nach den strukturrelevanten Funktionen des Raumes eine andere: Die Frage nach der Relevanz der Raumanalyse gesehen am Maßstab der Werkanalyse. Denn das Ergebnis der Gegenüberstellung dieser drei Vorfrühlingsszenen kann ohne weiteres formuliert werden, ohne daB der Begriff Raum dabei Verwendung findet. Dieses Ergebnis lautet folgendermaBen: An aufbaumäBig bevorzugte und bedeutende Stellen, nämlich an den Anfang, in die Mitte und an den SchluB des Werkes, stellt der Autor Szenen, die den Vorfrühling als Jahreszeit repräsentieren und durch merkmalhafte Gegenstände und Gegebenheiten sinnfällig vor Augen führen. An diesen Szenen demonstriert der Autor einen Sachverhalt von allgemein menschlicher Bedeutung, und zwar in Form antithetischer Reflexion: Im ersten Bild sind es Leben und Tod, die sich in der Geschichte von Mensch und Natur in stetiger Folge wiederholen. Im zweiten Bild sind es soziale Gegensätze, nämlich die Schicht der Armen und Unterdrückten im Gegensatz zum väterlichen Mythos der utopischen Glashäuser (S. $14 \mathrm{ff}$ ) und den darin beschriebenen glücklichen Menschen. Das dritte Bild verkörpert einen moralischen Gegensatz, indem der Frühling eine Dirne auf den Plan ruft, der Engel aber auch für sie die frohe Botschaft verkündet.

Hier findet man eine Anzahl von Einzelsymbolen: Das Grab symbolisiert den Tod, Blumen und Vögel das Leben. Dreck und Kälte symbolisieren eine niedere soziale Schicht, die Glashäuser den gesellschaftlichen Glückszustand. Die Dirne steht stellvertretend für die Sünder, der Glockenton für die Tugendsamen. Das zentrale Symbol ist aber nicht das jeweilige Bild oder die jeweilige Szene, die ich oben als HilfsgröBe eingeführt habe, sondern der Raum als Ganzes, als Schauplatz für die Austragung des Kampfes von Gegensätzen, der das Leben bildet. Dieses Symbol ist der Raum, und er trägt den Namen Vorfrühling. 
6. Die Erzählphase als homogenes Glied in der kontinuierlichen Genese der werkimmanenten Welt in Dąbrowskas "Noce 1 dnie"

Yit der Strukturcharakteristik "Bild einer Epoche" wird der Roman "Noce 1 dnie" von Maria Dąbrowska, erschienen 1923-34, in einen gattungsmäBigen Zusammenhang gebracht mit anderen Romanen, deren Zentralvorgang sich ebenfalls über einige Generationen erstreckt. Dies sind z.B. die "Buddenbrooks" von Thomas Mann, "Ies Thibaults" von Roger Martin du Gard, die "Forsytesaga" von Galsworthy, aus der polnischen Literatur die "Lalka" von Prus und "Nad Niemem" von Eliza Orzeszkowa. Am Beispiel der "Noce $i$ dnie" soll die Frage aufgerollt werden, wie das Bild einer Epoche im Zuge des Romangeschehens entsteht. Kann in Verlauf des Erzählvorgangs eine Sukzession festgestellt und herauskristallisiert werden, die in Aufbau eines Raumes ihr Ziel hat? Können die zeitlichen Verhältnisse des Romans Aufschluß geben über die Bedeutung räumlicher Funktionen?

Dabei kann von einer grundlegenden Beobachtung ausgegangen werden: Das Entstehen von räumlichen Wirkungen mit anschaulicher szenischer Geschlossenheit wird vermieden. Als Beispiel dafür soll die Szene dienen, welche einen Spaziergang der Heldin Barbara mit ihrem Sohn Piotrus durch eine Landschaft beinhaltet.

"Oft gingen sie einfach vor sich hin und schauten sich um, das war alles. Sie stiegen ein wenig auf die waldige Anhöhe, un dann auf einem schmalen Pfad, der sich durch ein farbenprächtiges Dikkicht dahinzog, wieder hinunterzugehen. Die vom Tau glänzenden Spinnweben kitzelten im Gesicht und die feuchten Blätter raschelten vernehmlich unter ihren Tritten und verbreiteten betäubende Düfte.

"Merkst du, wie die Blätter duften?" lieB sich die Mutter vernehmen; dann aber wandten sie sich um, der feuchte und schwarze pfad murde sandig, der Wald öffnete und lichtete sich, hier und dort zogen sich vertrocknete Heidefelder hin, und auf diesen muchsen Birken, deren intensiver Glanz, den sie um sich herum verbreiteten, die Augen blendete, so daB das Blau des Himmels dunkler erschien. 
"Dort, siehst du, wurde GroBväterchen Klemens krank", erklärte Frau Barbara, "dieser Platz heiBt Gryczól."

Manchmal hörten sie in der träumerischen Stille von der Höhe ein Klopfen, und dann verbargen sie sich und suchten wit angehaltenem Atem den Specht.

"Dort, dort," flüsterte die Mutter, starr auf den Kopf des Vogels blickend, der geschickt vom Stamm weghüpfte und deutlich auf dem Hintergrund des feuchten Himmels zu sehen war. Und sie nahm den Knaben vor sich oder hob ihn sogar ächzend in die Höhe, damit er dahin blickte, wo auch sie hinschaute.

"Dort," flüsterte sie, "auf dem zweiten Baum, auf dem zweiten, unter dem vertrockneten 7weig. Tiefer, tiefer. Siehst du? Siehst du, mein Söhnchen?"

"Siehst $d u$... Söhnchen," antwortete er hingerissen.

Ein anderes Mal trafen sie ein Eichhörnchen. Wie es flink und geräuschlos von einem Stamm zum anderen eilte und von Ast zu Ast,.. Bis schlieblich die Zeit kam, da alle Blätter abfielen, ihre letzten Dïfte hergaben und vermoderten, die Bäume aber zeigten ihre Schönheit von einer anderen Seite, eine gewissermaßen unvergängliche, durchgeistigte Schönheit der nackten Xste und Zweige ..." 1 Diese Szene aus dem Leben der beiden Figuren kann als Modellfall für den Aufbau der werkimmanenten Welt dienen. Sie bietet ein sehr anschauliches Bild, obwohl sie weder eine zeitliche noch räumliche Geschlossenheit aufweist. Die raumfüllenden Gegenstände, Blätter, Wald, Stämme, diste, Zweige, Heidefelder, Hügel, Spinnweben, tauchen nur in der Mehrzahl auf; sie sind von verschiedenen zeiten erlebt und entstammen nicht einem bestimmten, einmaligen Wahrnehmungsraum. Der zeitliche Ablauf ist ohne konsequente Reihenfolge, ja ohne Relevanz des Hintereinanders: Oft ... dann ... manchmal ... ein andermal ... Trotz dieser Irrelevanz der Reihenfolge tritt beim Leser keineswegs eine zeitliche Desorientierung ein, die Entwicklung läuft vielmehr im Sinne eines langsamen und stetigen Vertrautwerdens mit dieser Welt, so daß der Leser mit dem

1 Maria Dębrowska: Noce $i$ dnie, Warszawa 1953, Bd. 1, S. 79 f. 
Fortschreiten der Kapitel immer mehr von der mit dem Handlungsverlauf verbundenen Räumlichkeit wiodererkennt, sich zu erinnern glaubt, dort schon einmal gewesen zu sein.

An dieser Stelle soll der Begriff "Welt", wie er in der theoretischen Betrachtung des Romans immer wieder auftaucht, untersucht werden. Im Roman wird hinter dem Einzelnen, Privaten etwas Allgemeingültiges sichtbar, das Individuelle wird transparent und läßt Gegebenheiten großer, umfassender, totaler Ausmaße durchscheinen. Diesem Umstand versucht die Analyse durch den Begriff Welt gerecht zu werden: Der Roman ist eine Welt für sich, im kleinen.

Frank 0. Maatje sagt in seinem "Versuch einer Poetik des Raumes" 1 folgendes: Die Welt im sprachlichen Kunstwerk wird durch die Lese-, Rezitier- oder Aufführungszeit konstituiert ... Dichtung ist nur insofern "Zeitkunst", als sich die Realisierung des vom Dichter Geschriebenen erst in der Zeit der Lektüre, der Rezitation oder der Aufführung vollzieht... Die Welt, die Zeit-Raumstruktur des Sprachkunstwerkes, existiert einfach nicht, wenn keiner, kein Leser, kein Vortragskünstler, kein Schauspieler das Werk aktualisiert. Die Welt-im-Werk ist nicht dauernd und in concreto gegenwärtig." 2

Hier hat das Subjekt Welt drei Prädikate: konstituieren - relisieren - aktualisieren. Wenn es auch schwer ist, sie bedeutungsmäBig genau zu fixieren und gegeneinander abzugrenzen, so zeigen doch alle drei, daß es sich um Vorgänge handelt, deren genauer Verlauf durch die zeitliche Struktur des Werkes bestimmt und gelenkt wird. Diesen ProzeB, den ich Raumgenese nenne, kann man im Roman "Noce $i$ dnie" aufs genaueste verfolgen.

Die Naturgegebenheiten, die den Schauplatz bilden, sind, wie die überwiegende $\mathrm{Zahl}$ der Verben zeigt, mit einer Bewegung versehen. Sie verlieren dadurch ihre feste und einmalige Gestalt, was noch unterstrichen wird durch ihr Auftauchen in der Mehrzahl: Blätter, Stämme, Äste, Zweige, Heidefelder, Hügel, Spinnweben. Aber es ist noch eine andere Erscheinung, die nicht einen einmaligen, ad hoc

1 In: ZRL 11 (1968), Heft 1 (20), s. 5-23.

2 A.a.0., S. 5 ff. 
vorliegenden Wahrnehmungsraum entstehen lassen. Der Vorgang, der sich auf diesem Schauplatz abspielt, zeigt keine in sich geschlossene Handlung. Deren zeitlicher Ablauf ist ohne konsequente Reihenfolge, ohne Relevanz des Hintereinander. Seine genaue Fixlerung in dieser Hinsicht wird geradezu unmöglich gemacht durch die Temporalangaben: oft ... dann, manchmal, ein andermal. Trotz dieses Verzichts auf zeitliche Orientierungshilfen tritt beim Leser keineswegs eine Desorientierung ein, er wird vielmehr im Laufe des Weiterlesens immer vertrauter mit dieser Welt, so dab er mit dem Fortschreiten der Kapitel immer mehr von der mit dem Handlungsverlauf verbundenen Räumlichkeit wiedererkennt, sich zu erinnern glaubt, dort schon einmal gewesen zu sein.

Die konsequente Auflockerung des zeitlichen Zusammenhalts von Vorgängen ist nicht nur Merkmal dieses Spaziergangs, dessen Verlauf ohnedies nicht zielgerichtet und damit abhängig von einer bestimmten Reihenfolge ist. Als ein weiteres Kapitel kann der Anfang des zweiten Kapitels dienen, wo unter der Uberschrift: "Wieczne martwienie", "endloser Gram", eine Schilderung der Kinderwelt vorliegt. Lose Szenen sind aneinandergereiht in Form der direkten Rede, ohne Zusammenhalt eines einheitlichen Schauplatzes, aber zusammengehalten durch den Strom der Schilderung und Erzählung. Dies verdeutlichen die jeweiligen Anfänge der Absätze: "Ein andermal fragte sie den Mann ..." (S. 172 f.) "In einem Frühling ..." (S. 173 ff.) "Eines Tages ..." (S. 175) "Als die herbstlichen und winterlichen Fröste hereinbrachen ..." (S. 175) Es handelt sich wohl um Ausschnitte, wenn man eine chronometrische Zeitlinie zugrunde legt, doch kann man diese Zeitbehandlung nicht als Raffung bezeichnen, da ja, zumindest im kleineren $\mathrm{Zu}-$ sammenhang, keine zwingende zeitliche Konsequenz beobachtet wird. So erhalten diese Glieder den Charakter von Beispielen, welche erläutern und anschaulich sichtbar machen, wie es in dieser Welt aussioht. Es tauchen Personen auf und werden auf das genaueste charakterisiert, und sie können wieder abtreten, ohne im Gesamtverlauf der Handlung eine entscheidende Rolle gespielt zu haben, wie z.B. das Kindermädchen Jozia, von dem es bei ihrem Verschrinden vom Schauplatz heiBt: "Und so verschwand ihre Spur, und die Angelegenheit versank in tausend anderen, die herankommen, sich 
drängen, die Gedanken in Anspruch nehmen, den Schlaf von den Augenlidern vertreiben und dann vorübergehen, aber den Lauf des menschlichen Lebens unmerklich lenken." (S. 176)

Diese Diskontinuität des erzählten Vorgangs - wobel Vorgang hier nicht als wissenschaftlicher Terminus zu betrachten ist, sondern als vom Autor konzipierte Handlung mit festliegendem Ablauf wurde schon in Sienkiewicz' "Quo vadis" festgestellt und die damit verbundene Aufrechterhaltung der räumlichen Wirkung nachgewiesen. Doch handelte es sich dort um die Unterbrechung dieses Vorgangs, während im vorliegenden Roman eine in dieser Form konzipierte Handlung nicht faßbar wird. Dafür ist der Verzicht auf zeitliche Orientierungshilfen verantwortlich. Dieser Mangel an zeitlicher Orientierung wird aufgehoben durch das Entstehen eines Raumes, nicht des Ortes der einzelnen Handlungsabschnitte, sondern eines übergeordneten, der vom Leser miterlebt wird und der inm das Erlebnis einer illusionsmäßigen Versetzung in eine geschlossene Welt oder in eine bestimmte historische Epoche vermittelt.

Deshalb erfahren die im Verlauf der einzelnen Erzählphasen auftauchenden Handlungsabschnitte keinen konsequenten Abschluß. Sie bilden aber eine Komponente des Raumerlebnisses. Keines dieser Glieder zeichnet sich vor den anderen durch eine besondere, betont räumliche Wirkung aus. Die Intensitätskurve dieser Wirkung kennt kein Auf und $\mathrm{Ab}$. Durch die Aufrechterhaltung der Wirkung über die wechselnden Ereignisse durch einen großen Zeitraum hindurch entsteht eine Welt, "ein breites Bild des Lebens einer ganzen Epoche, imponierend ebenso durch die Breite des historisch gesellschaftlichen Hintergrundes wie durch die Eindringlichkeit und Tiefe des Blicks auf den Menschen, durch Wahrhaftigkeit und Reichtum der Charaktere." 1

1 Matuszewski, R.: Literatura polskaw latach 1918-1955, Warszawa 1961, S. 111. 
7. Sukzessive Differenzierung des fiktiven figuralen Wahmehmungsfeldes und Genese der Momentsituation. Entmedialisierung der Sprache in Iraszkiewicz' "Młyn nad Utratą"

Jarosław Imaszkiewicz leitet seine Novelle "Die Mühle an der Utrata", geschrieben 1936, in Ichform ein, wobei er den fiktïven Charakter der Erzählung hervorhebt. Auf einem Dorffriedhøf in der Nähe von Warschau hat er einen Grabstein entdeckt, wellcher das Grab von zwei Freunden ziert, die kurz hintereinander gestorben sind. Ihre Namen sind ihm bekannt, sein Interesse erwacht, er stellt Nachforschungen bei Frounden und Bekannten dier beiden an und versucht, aus den bruchstückhaften Nachrichten ihren Schicksalsverlauf und die Entwicklung ihres Verhältnisses zueinander zu rekonstruieren. Die Konfliktsituation dieser Fiiguren und ihre Entwicklung ist Gegenstand der Darstellung. Wesentliche Komponenten dieser Situation werden von räumlichen Elementen gebildet, d.h. sie werden an landschaftlichen Bildern so demonstriert und verdeutlicht, daß die augenfälligen Handlungen und die damit verbundenen Sprechakte nur im durch sie gebildeten Umfeld ihre Bedeutung erfüllen und ihren Sinn erhalten.

Der Aufbau des Werkes erfolgt nun nicht so, dab zu der dramatischen Entwicklung der beiden Helden und ihres Verhältnisses ein Raum in Form eines Schauplatzes hinzufingiert wird, etwa mit dem Ziel, die Anschaulichkeit zu erhöhen und den Vorgang plastisch vor Augen zu führen, also beim Leser die Illusion zu erzeugen, er wäre als Augenzeuge beim Geschehen anwesend. Ein solcher Schauplatz ist wohl vorhanden und wird auch beschrieben, doch erschöpft der Raum seine Funktion nicht als schauplatzmäBiges Umfeld. Die Verönderungen in der Persönlichkeit der Helden und die Entwicklungsprozesse werden in einer Reihe von repräsentativen Situationen beleuchtet, an deren räumlicher Komponente dfe Fiktion ansetzt. Die Berechtigung, diesen Raum zu beschreiben und zu analysieren, liegt nicht darin, daB dieser mit all seinen Merkmalen und Eigenschaften Aufschluß gibt über die Figur und inren augenblicklichen Zustand, so daB der Autor, hätte er mehr Einzelheiten über das Leben der Helden gemußt und erfahrez 
odier iäre er gar als dritter dabeigewesen, den Raum nicht nötig ge:habt hätte. Die Situationen als Entwicklungsphasen der Figure:n sind vielmehr selbst räumlicher Natur und bilden in ihrer Abifolge eine wesentliche Komponente im konsequenten Verlauf diese:r Entwicklung. Diese könnte also mit dem Vokabular der psychisc.hen Analyse beschrieben werden, doch wïrde dadurch der strukturelle Bereich der Erzählung durchbrochen.

Dais Wechselverhältnis von seelischem Zustand und räumlicher Situation zeigt etwa folgende Stelle: "Weil er vor Zorn nicht wuBte, was er tun sollte, trat Karol auf den Balkon hinaus. Im Augenblick des Sonnenunterganges wurde der halbe Himmel plötzlich rot, purpurfarbene Aderchen flogen über den grauen Grund, und alles vor der Vorlaube, Ulmen, Gebüsch und die zwei roten Wirtschaftsgebäude, alles erstarrte in diesem Schein. Ställe und Scheunen standen im Dickicht versunken wie Schiffe im Schlamm eines grünen Kanals. Und in diesem gewöhnlichen, alltäglichen Anblick bemerkte Karol eine Bewegung oder die Möglichkeit einer Bewegung. In Kauern der Gebäude, in der Neigung der wilden Fliedersträuche spürte er etwas wie Erwartung." 1 Man kann nicht sagen, daß der Zorn sich in irgendeiner Form auf das räumliche Bild überträgt, so daß jeder Komponente des Bildes ein konstituierendes Moment des figuralen Seelenzustandes entspräche. Der Augenblick der Erregung wird auf den Raum projiziert; vom in diesem Augenblick erlebten Wahrnehmungsfeld der Figur werden einige Stellen, deren Momentancharakter besonders augenscheinlich ist, ausgewählt, mit einem Symbol versehen und zu einem Erzählvorgang zusammengefaßt. Durch diese Zergliederung des momentanen Zustands wird die Ubertragung der räumlichen Wirkung auf den Leser geleistet. Der Raum der Figur wird in dessen Illusion aufgebaut und gestaltet.

Auf den Raum wird an besonderen Stellen des dramatischen Vorgangs eingegangen, ohne daß das Ziel die Erfassung und Differenzierung dieser Besonderheit wäre. "Dieser Kuß tauchte die ganze Landschaft in eine andere Farbe. Die paar Kiefern, der Teich, die Wihle, sogar Karols Gesicht - sahen anders aus." (S. 307) Weder

1 Jarosław Imaszkiewicz: Opowiadania 1918-1953, Bd. 1, Warszawa $1956, \cdot S .310 \mathrm{f}$. 
wird gesagt, was an dem KuB Besonderes war noch in welchen Erscheinungen sich die räumlichen Gegebenheiten verändert haben.. Die AuBergewöhnlichkeit bildet eine eigenständige Situationskomponente, die für die Bedeutungserfüllung der weiteren Situationen und der damit verbundenen Symbole die Feldwerte bereiitistellt.

An den letzten Beispielen tritt deutlich zutage, daB der Farbue beim Aufbau dieser Räume eine große Bedeutung zukommt. Farbem sind mit jeder räumlichen Situation der Novelle verbunden, doch sind es nicht die Farben an sich, welche am Aufbau beteiligt sind, sondern jedesmal ganz außergewöhnliche Farbtöne, welche die Einmaligkeit und Merkwürigkeit der Situation hervorheber. in vielen Fällen sogar ausmachen. Die Nennung der Farben entspricht keiner Differenzierung des Wahrnehmungsraumes, sonderm einer wesentlichen Komponente des Situationsfeldes unter dem Aspekt des Momentancharakters. Für diese Farbtöne einige Beispiele: "So wuchsen die Blumen, wie Gott es fügte, und erfül1ten die Vertiefung um den Teich mit einem grünrosa Glanz." ( $S$. 271) "Gegen den aprikosenfarbenen Bezug hoben sich sehr deutIich sein grünlicher Teint und das aschgraue Haar ab. Die breiten schwarzen Brauen hatte er mit dem Ausdruck von Unruhe und Ermïdung zusammengezogen. Er blickte starr auf einen kleinen silbernen Gegenstand." (S. 277) Hier wird sogar deutlich, daB die Situation nicht aus Komponenten schauplatzmäßiger Art zusammengesetzt und aufgebaut ist, sondern der Autor erwähnt nur Elemente, die sich durch einen besonderen Farbton auszeichnen. "Im Salon, inmitten der weiBroten Kretonnebezüge der alten Möbel und der grünlichen Spiegel mit den stark vergoldeten Rahmen stand Desmond King." (S. 289)

Immer sind die Landschaftsbilder von solchen Farbtönen bestimmt, die nicht in ihrem simultan wirksamen Zusammenspiel harmonisch wie in einem Bild aufeinander abgestimmt sind, sondern in ihrem Charakter als $\mathrm{Zwischentöne} \mathrm{übereinstimmen.} \mathrm{Die} \mathrm{Bedeutungserfül-}$ lung der Zeichen, die einen solchen Farbton andeuten, erfolgt nicht durch die Zusammenschau mit anderen Farben in einem Bild, sondern im zeitlichen Feld des Lesevorganges, wo sie einen Wert 
darstellen und so mitwirken an der Konstitution einer momentanem Impression. "Hinter dem grünen Wäldchen kroch an dem vor Hitze leicht erblaBten Himmel langsam eine runde, GroBe Wolke hervor, die mit ihrem Violett ein Gewitter ankündigte." (S. 290) "Und mit einer beredten Geste zeigte er auf die in diesem Augenblick schwarze Strömung, die unten dahinrauschte, tintenfarben mit dem ersten grünen Glitzern. Karol war augenblicklich nüchtern und faßte den Kommissar unterm Arm." (S. 293) Die Wirkung dieser Bilder beruht nicht auf der Komposition von Griin und Violett bzw. Grün und Schwarz, sondern auf dem konzentrierten Stimmumgserlebnis, welches seine Gespantheit durch das fluktuierende, schwer fabbare Wesen der Farbe erhält. Noch deutlicher ist dies in folgendem Beispiel ausgefüht. "Vor Abend brachen die Preunde nach Hause auf. Es war eine warme masowische Dämmerung, die Ränder des Himmelsgewölbes verschmolzen in einem leichten blauen Nebel mit dem Horizont. Uber die Wiesen, auf denen riesemgroße Störche standen, strich ein kühler, geheimnisvoller Hauch, als sickerte die kalte Luft von irgendwoher in schmalen, verzweigten Bächen herein. Der Klee durftete stark, die Grillen zixpten darin in unerhörter Anzahl. Die Felder wirkten wie mit einer dünnen Schicht dieses Gezirps überzogen. An dem Ort, wo der verwischte Nebeldunst des Horizonts in die grünliche Kuppel des Firmaments überging, steckte ein kleiner Mond, ähnlich einem obgeschnittenen Fingernagel. Und dann fuhren sie bereits in den Garten ein, zwischen Bohnen, Rosen und Levkojen." (S. 308) Diese merkwürdige Verschwommenheit und Unklarheit des Eindrucks kann nicht zurückgeführt werden auf die Unfähigkeit des Autors, cen dem Auge sich bietenden Gegebenheiten mit den Mitteln der Sprache gerecht zu werden. Die Grillen in unerhörter Anzahl, der abgeschnittene Fingernagel und die unklaren Positionsangaten haben sich vom handgreiflich Gegebenen abgelöst und bilden ein selbständiges Feld, für dessen Stellen nicht die Zugehörigkeit zu einem bestimmten Raum relevant ist, sondern ihr Zusamnenwirken zu einem spezifisch neuen, überraschenden Erlebnis, as einer Situation mit Augenblickscharakter. Die Sprache dient richt als Medium, eine vorliegende Situation zu erfassen, sondern erst im sprachlichen ProzeB wird die Situation manifest. In dieser Hinsicht wird die Situation zu einer strukturellen 
Einheit, da sie sowohl dem erzählten Vorgang als auch dem Erzählvorgang und dem korrespondierenden Leseerlebnis zugeordnet werden kann.

Die Abhängigkeit der Erlebnissituation von ihrer sprachlichen Realisation zeigt sich besonders deutlich dort, wo sie das Bewußtsein der eigenen Existenz betrifft und so von der Figur selbst formuliert wird. Hier tauchen eine Reihe von Bildern auf, bei denen die Konsistenz und Körperlichkeit der Wahrnehmungsdinge von der Figur in Zweifel gezogen werden. Sie erhalten dadurch den Charakter von etwas Künstlichem, ihre Eigenschaften erschöpfen sich nicht im sinnlich Gegebenen, sondern beruhen auf einer Interpretation, die den Grad ihrer Realität untersucht. "Angst und Unruhe packten ihn, er öffnete das Fenster, aus dem er auf die Blechdächer der Nachbarhäuser hinaussah, und ihm war, als wohnte er in solch einer grauen, blechernen Welt, wo die verbogenen Metallsilhouetten so tun, als flösse durch sie der Strom des Lebens. Ein weißgrauer Himmel tat sich vor inm auf, künstlich und unwirklich." (S. 296) Der räumliche Aufbau, die räumliche Ordnung werden dadurch erschüttert, daß das Material der Dinge seiner Zuverlässigkeit und Stabilität beraubt wird. Die existentielle Unsicherheit findet ihren Ausdruck darin, daß die betreffende Erlebnissituation der Figur in bestimmten Augenblicken einen Höhepunkt, eine momentane Konzentration erreicht. Dann wird von den in dieser Situation vorhandenen raumfüllenden Gegenständen das besonders gesehen und vom Autor ausgewählt, was seiner Natur nach vergänglich und unsicher ist, oder es wird diese Eigen. schaft den jeweiligen Elementen verliehen: "Und in diesen grünen Landstrichen steckten graue Berge und gläserme, gleichsam versehentlich hingestreute Seen ... Der unablässig blaue Himmel hing tief über den Bergen, die wie aus Stoff oder dichtgewebter Seide gemacht sdhienen." (S. 327) "Es war ein schöner, sehr warmer Maitag. Der Himmel füllte sich mit großen Wolken; sie standen hoch und von der Sonne beleuchtet wie Modelle unirdischer Berge. Flüchtig und weiß veränderten sie ihre Gestalt, wïhrend sie durch das nicht allzu grelle Blau wanderten. Der Teich glitzerte sauber und bläulich." (S. 353 f.) Strukturell bedeutsam ist die erlebnismäBige Intensität, welche durch die ständige 
Wiederholung von Bildern dieser Art aufrechterhalten wird, wodurch der ganze Schauplatz der figuralen Handlung in dieses unsichere licht gerückt und in Mitleidenschaft gezogen wird. Nicht das imaginäre, flüchtige und keinen Halt bietende Raumbild wird dadurch repräsentativ für diese Welt, sondern die Möglichkeit, daß jedes Bild als Zugang zu dieser Welt einen solch unsicheren und unwirklichen Charakter erhält.

Dem Aufbau der Novelle kommt man nicht näher, wenn man die Techniken, mit welchen der reale Zusammenhalt des räumlichen Bildes zur Auflösung gebracht wird, vollständig sammelt und in ihrer Verschiedenartigkeit gegenüberstellt. Man muß analysieren, wie der dramatische Handlungsverlauf vom Charakter dieser Bilder gelenkt und bestimmt wird.

Die Novelle endet mit dem Tod der beiden Helden. Dieser Tod steht schon von Anfang an fest, ihre Grabsteine bildeten ja die Einleitung. Aber die einzelnen Handlungsabschnitte haben für sich betrachtet keinen Zusammenhang mit dem Tod und führen in keiner Weise mit folgerichtiger Konsequenz auf dieses Ende hin. Man kann im Verlauf des Geschehens kein Motiv entdecken, welches auf den Tod hinweist. Der Erzählung eines zufälligen Bekannten vom Selbstmord seiner Frau steht das Erlebnis der Geburt gegenüber; auf diese Weise motiviert der Autor die Frage, welche die Figur Karol das ganze Leben hindurch beschäftigt: "Was ist der Mensch?" Das Problem ist: Läßt sich trotz dieses unmotivierten Todes eine Entwicklungslinie herausschälen, als deren Endglied dieser Schluß angesehen werden kann?

Einen Hinweis gibt hier eine Gesprächsszene zwischen dem Helden Julek und einer Randfigur, dem Neger Desmond King. "Sie sind ein Dichter - sagte er zu Julek und sprang ein wenig in die Höhe wie ein ungefiederter Hahn, - Sie wissen es! In der Poesie gibt es solch einen Augenblick, in dem Sie plötzlich für eines Lidschlags Dauer eine andere Welt erblicken. Und Sie sehen auch, daß alles nur ein Schatten jener anderen, wirklichen Welt ist. So wie im Gebet. Sie sprechen die einfachsten Worte von der Welt, wie jene berühmten Worte:

All night have the roses heard

The flute, violin, bassoon ... 
und plötzlich fühlen Sie ein Zittern, Sie sehen die Rosen, aber nicht die, welche vor dem Fenster blühen, sie hören Flöten und Fagotte, aber nur jene wahrhaften, wie bei Giotto. Ja, ja, das 1st banal, was ich spreche, banal, aber wahr, banal, aber wahr..." (S. 303 f)

Dieses Augenblickserlebnis ist ein Leitmotiv der Novelle. Und wie man auf der einen Seite den Eindruck hat, man müßte nur lange genug das räumliche Bild betrachten, um seiner Unwirklichkeit auf die Spur zu kommen, gibt es Augenblicke, in denen eine andere Wirklichkeit blitzartig aufleuchtet und das eigentlich Gesehone in den Bereich der Unmirklichkeit rückt. Der Charakter der räumlichen Situation ist bestimmt durch die Möglichkeit eines solchen Augenblicks, welcher nicht selber erscheinen muß. "Er schaute stumm auf diese rote Beleuchtung, und für einen Augenblick schien es ihm, als wïrde die Landschaft ihm ihr Geheimnis enthüllen, wenn er nur ein Wort spräche. Aber der Augenblick der Erwartung verging, die Frage, die Karol in der Tiefe des Geistes verbarg, kam nicht an die oberfläche und er erhielt keine Antwort. Der Vorhang der Maja bebte gleichsam, und dann erstarrte er sofort." (S. 311) Hier besteht die Bedeutung des auktoralen Sprechaktes in der Transposition der Situation von der Figur auf den Leser, wobei der Charakter der Situation den Ausschlag gibt und nicht die Differenzierung der einzelnen Komponenten. Im Mitelpunkt des Beschreibungsvorgangs steht der Augenblickscharakter, auf inn wird jedesmal bei seinem Auftauchen hingewiesen, das Auftauchen des Augenblicks wird vom Autor szenisch vorbereitet. Die Augenblicke in ihrer Gesamtheit konstituieren eine Folge, welche alle dramatischen Verlaufseigenschaften in sich birgt und mit dem Ende der Novelle einen folgerichtigen AbschluB erhält. Die Veränderung, die dabei zwischen den einzelnen Momenien stattgefunden hat, wird zwar häufig erwähnt, wie z.B. an dieser Stelle: "Und wieder befand er sich am Teich, ungefähr an der gleichen Stelle wie vor einigen Tagen, wieder sah er von ferm. wie Desmond die Angel in das von grüner Entengrütze bedeckte Wasser auswarf, es näherte sich sogar wieder ein Gewitter ode: schlechtes Wetter, und doch war der innere Augenblick ein völigg anderer." (S. 300) Der Abstand, der zwischen diesen Augenblichen 
Liegt, ist nicht zu messen mit der Zeit, welche den Ablauf der Hilandlung bestimmt, etwa den Verlauf des Konfliktes zwischen den beiden Freunden. Das Augenblickserlebnis ist in keinem Falle ursächlich mit diesem Geschehen verbunden. Der vordergründige Fhasenablauf mit der Richtung auf die Lösung des Konfliktes zwischen den Helden ist ein homogenes und gestaltloses Geschehen, welches an sich keinen bestimmten AbschluBpunkt anstrebt und auch keinen erhält, sondern nur die Grundlage für die Herausbildung von Phasen bildet.

So ist der Schlußpunkt auch nicht der Tod, sondern der Todesaugenblick, in welchem Karol das angestrebte Ziel, den Anblick der eigentlichen Realität, erreicht: "In letzter Anstrengung drehte er sich auf den Rücken und sah über sich eine hohe weiße Wolke und die violetten Querwölkchen davor und den Himmel ohne Grund und Ende, der im Blau verschmolz. Das dauerte kaum den Bruchteil einer Sekunde, aber er fühlte und verstand all das, was er Zeit seines Lebens nicht verstanden hatte, und vor allem jene Nacht in Werfen. Der Vorhang der Welt wich, zerriB, verschwand, und er sah - nein, er sah nicht einmal - sondern er berührte mit den Fingern, durch die sich das farblose Wasser ergoB, das lebendige Geheimnis, welches für ihn schon aufhörte, ein Geheimnis zu sein. Es war nur die Süßigkeit ohne Maß und die große Ruhe, von der nur der Tod einen Begriff gibt." (S. 356 f.) Karois Freund ist schon vorher gestorben, und zwar unter ebenso unglücklichen Umständen, wie Karol seinerseits das ersehnte Glück erlebt. Darauf beruht der gegenläufige Bewegungsaufbau der Novelle: Ein gläubiger und ein ungläubiger Mensch bewegen sich, was den Grad ihrer existentiellen Sicherheit betrifft, aufeinander $z u$ und entfernen sich mit umgekehrten Vorzeichen wieder voneinander. 
8. Der thergang des Zeichenkontextes in eine selbständige räumliche Situation in Bruno Schulz' "Sklepy cynamonowe" und "Emeryt"

Der Raumaufbau in Werk Bruno Schulz' bietet der Analyse die beesondere Schwierigkeit, daß der Autor selber nur interpretieremderweise auf den Raum eingeht. Es läßt sich kein einheitlicherr, lokalisierbarer Autorenstandpunkt feststellen, da dieser sich ausschlieBlich im sprachlichen FormulierungsprozeB manifestiert und sich in ständiger Bewegung befindet. So bildet jeder Satz eine neue Situationskomponente, die für die Bedeutungserfüllumg des folgenden Satzes relevant ist; dieser Satz wird wiederum Ausgangsbasis für den folgenden.

Der Autor selbst verwendet beim Erzählen vorzugsweise Begriffe der interpretierenden Terminologie. Er erscheint in der Ichform als eine Figur, die an den stattfindenden Ereignissen nicht alktiv beteiligt ist, sondern als beobachtender Kommentator danetbensteht. Der Literaturwissenschaftler kann also nicht seinerseits die vorgefundenen Phänomene analysieren und interpretieren, olhne daß sich seine Funktionen mit der des Ichautors überschneiden würden. Diese terminologische Grundschicht des beim Erzählen verwendeten Wortmaterials bildet also das primäre Objekt der wissenschaftlichen Analyse. Die Tatsache, daß neben dem betont distanzierten Kommentator eine Fiktion von Räumen, Figuren und Vorgängen als Ergebnis dieser Art von Erzählen vorliegt, wie sie an Ungewöhnlichkeit und Phantastik kaum zu überbieten ist, ist die spezifische Eigenart in Schulz' Werk. Die Art, wie diese beiden sich ausschließenden Gegebenheiten, die Beschreibung von etwas konkret Vorliegendem und die Fiktion von vollständig Neuem, Einmaligem, nebeneinander existieren und zu einem einheitlichen Erzählvorgang verbunden werden, sei vormeweg folgendermaßen umrissen:

Die verwendeten interpretativen Termini, deren Wesen es ja ist, stets den gleichen, fest umrissenen und vorher festgelegten Sachverhalt $z u$ betreffen und einer bestimmten Kategorie anzugehören, werden vom Autor in einer abbildenden Zuordnung realisiert und 
erlebt. Der wahrgenommene Gegenstand wird als solcher genannt, doch er wird bereits im Augenblick der Nennung in einen dynamischen sprachlichen EntfaltungsprozeB hineingezogen. Damit wird seine anschauliche Realisation in Dimensionen verlagert, die über seine ursprünglichen Eigenschaften und den ursprünglichen semantischen Feldwert des Begriffs weit hinausgehen. Diese Erscheinung bezeichne ich mit "semantischer Perspektive".

In einem Kapitel der "Zimtläden", welches mit "Pan" betitelt ist, findet sich die Schilderung eines Gartens. Dieser ist zunächst angefüllt mit Wahrnehmungsobjekten, wie man sie in einem Garten erwartet: Birnbäume, Apfelbäume, Gras, Ährenbüschel, Petersilie, Möhre, Efeu, Taubnessel, Wegerich. Stellt man sich jedoch nur diese Gegenstände allein zu einem Garten zusammengefügt vor, so ist das Ergebnis durchaus nicht gleich dem, welches durch die entsprechenden Sätze im Zusammenhang der Erzählung entstanden ist. Hier heißt es: "Dort war ein großer, verwilderter, alter Garten. Hohe Birnbäume, ausladende Apfelbäume wuchsen dort in selten mächtigen Gruppen, überschüttet mit silbernem Rascheln, mit einem brodelnden Netz weiBlichen Glitzerns. Uppiges, vermischtes, nie gemähtes Gras bedeckte mit flaumigem Pelz das Terrain. Es gab dort die gewöhnlichen Wiesengrashalme mit gefiederten Ährenbüscheln; es gab die zarten Filigrane der wilden Petersilie und der Möhre; die runzligen und rauhen Blättchen des Efeus und der Taubnessel, die nach Minze dufteten; die bastartigen, glänzenden Wegeriche, vom Rost gesprenkelt und in Rispen dicken roten Breis emporschießend." (S. $83 \mathrm{f.}$ )

Hier sind keine weiteren raumfüllenden Gegenstände zu den ursprünglichen Gartenrequisiten hinzugetreten, wohl aber eine große Anzahl von weiteren Worten, die für den Charakter dieses Raumes sehr viel bedeutsamer sind. Die Zeichen für die Requisiten bilden nur ein Gerüst, welches eine um vieles reichhaltigere und ajwechslungsreichere Anzahl von Worten stützt. Diese betreffen dle Konsistenz der Requisiten, ihre Oberfläche, wie sie sich dem Tastsinn bietet, und verleihen ihnen etwas Auffallendes, ein über das Pflanzliche hinausgehendes Leben. Dann geht der Autor auf den dzrüber befindlichen Luft- und Himmelsraum ein. Daß dieser aller- 
lings darüber befindlich ist, kommt nicht zum Ausdruck, vielnehr vermischt er sich mit der Pflanzenwelt. Er ist nicht ein leerer, unendlich weiter Raum, sondern hat gegenständlichen, nandgreiflichen Charakter. "Dies alles, filzig und flaumig, waar nit milder Luft durchtränkt, mit blauem Wind unterfüttert und nit Himmel angefüllt." (S. 84) Der Standpunkt des Autors ist nicht mehr der des beobachtend Wahrnehmenden, sondern sein Staandort wird ein Teil dieses Raumes, der mit den raumfüllenden Geggenständen in direkter Berührung steht: "Wenn man sich ins Gras Idegte, war man bedeckt von der ganzen blauen Geographie der Wolkeen und der schwimmenden Kontinente, atmete man die ganze weitläuffige Landkarte des Himmels." (S. 84) Die Gegenstände des Raumes verwandeln sich in Materie, die sich vermischt und durchdringtt: "Von diesem Umgang mit der Luft bedeckten sich die Blätter und 1 Triebe mit zarten Härchen, mit einem weichen Anflug von Flaum,, mit rauhen Borsten und Häkchen, wie zum Einfangen und Festhal-ten des vorbeifließenden Sauerstoffs. Dieser zarte und weiBlicche Anflug verschwägerte die Blätter mit der Atmosphäre, gab ihm dden silbergrauen Schimmer von Luftwellen, von schattigen Grübeleieen zwischen zwei Sonnenblitzen." (S. 84)

Nun ist eine neue Art von Raum entstanden, das visuelle Nomentt ist dem des. Tastens gewichen. Das Ergebnis dieser Berührungen wird wieder Gegenstand der Betrachtung, aber in einer Form, wiie sie nur durch die Vermischung der Sinneswahrnehmungen zustande 3 kommen konnte. Es taucht jetzt eine neue Pflanze auf, die sich 1 nicht in die traditionellen botanischen Kategorien einordnen läBt, sondern der Umwandlung des Luftraumes in einen Körperraum entspringt: "Doch eine dieser Pflanzen, gelb und voll milchigeen Safts in den blassen Stengeln, mit Luft aufgeblasen, trieb auas ihren leeren Trieben nur eitel Luft, eitel Flaum in Gestalt ge:fiederter Milchkugeln, die im Windhauch zerfielen und sich lauztlos in die blaue Stille saugten." (S. 84) Zwar läßt sich in dileser Pflanze unschwer der Löwenzahn wiedererkennen, doch sind seine Dimensionen dermaßen erweitert, daß er nur mit den Kate-gorien von Himmel und Erde erfaßt werden kann. Jedes einzelne der Elemente, aus denen sich der Garten zusammensetzt, wird zum konstituierenden Glied einer Welt, einer Totalität. 
Wenn die Analyse sich immer wieder der Wendung bedient: jetzt ist dies oder das geschehen, so soll damit zum Ausdruck gebracht werden, daß eine Entwicklung schrittweise verfolgt wird. Dabei mu beachtet werden, $d a B$ auch der Autor eine Fntwicklung am Darstellungsobjekt aufzeigt, und daß die Sätze der interpretierenden Analyse oft in gleicher Form auch vom Autor selbst geprägt werden. Daß beispielsweise der Garten sich als Raum in den Kategorien universaler und weltweiter Dimensionen bewegt, wird auch vom Autor selbst gesagt, und zwar dort, wo er den Weg beschreibt, auf dem man in den Garten eindringen kann: "Es war das entfernteste Kap, das Gibraltar dieses Hofes, das verzweifelt mit dem Kopf gegen den blinden Plankenzaun aus waggrechten Brettern schlug, welcher auch die letzte Wand dieser Welt abschloB ... Mit einem FuB auf dem Brett stehend, das wie eine Brücke über die Pfütze gestürzt war, konnte sich der Gefangene des Hofes in waagrechter Haltung durch den Spalt zwängen, der ihn in eine neue, luftige und weite Welt hinauslieB." (S. 83)

Während die Analyse das vorgefundene Material zu ordnen und zu differenzieren versucht und sich deshalb abstrahierender Begriffe bedient, die einen Komplex zusammenfassen und so immer wieder dem gleichen räumlichen Tatbestand zugeordnet sind, verwendet der Autor die nämlichen Begriffe, un alle Möglichkeiten ihres Bedeutungsbereiches sprachlich zu realisieren. Indem er diese Köglichkeiten bis an ihre Grenzen ausschöpft, gelangt er schließlich zu räumlichen Gegebenheiten, die mit der Natur des ursprünglichen Darstellungsobjektes keine Verbindung mehr haben, sondern einen neuen, eigenständigen Kosmos bilden. Nach der Darstellung der überdimensionalen Pflanze setzt eine Raumfiktion ein, in welcher der tbertragungsvorgang von gegenständlichen Elementen in die Sphäre der Totalität, des weltweiten Aspekts bereits vollendet ist und der Standpunkt des Autors sich im Erleben universaler Kategorien bewegt: "Der Garten war ausgedehnt und in einige Seitenarme verästelt und besaß verschiedene Zonen und Klimate. Auf der einen Seite war er offen, voll Milch des Himmels und der Luft, und dort war der Himmel mit dem allerweichsten, allerzartesten Grïn unterpolstert. Doch im gleichen Maße wie der Garten in die Tiefe eines langen Seitenarmes abfiel und sich in den 
Schatten zwischen die Rückwand einer verlassenen Sodawasserfabrik verkroch, verdüsterte er sich merklich, wurde er aufbrausend und nachlässig, lieb sich wild und schlampig die Haare wachsen, wütete in Brennesseln, sträubte sich in Disteln, räudete sich in allerhand Unkraut, bis er ganz am Ende zwischen dien Wänden, in einer breiten und rechtwinkligen Bucht, alles MaB verlor und in Wahnsinn verfiel." (S. 84) Das räumliche Situatiionsfeld hat sich in den Bedeutungsbereich krankhafter menschlicher Emotionen verwandelt. In diesem findet die weitere Entwicklung statt, in diesem bewegt sich der weitere Erzählvorgang, in dessen Verlauf alle Merkmale und Eigenschaften eines Typs auftauchen, der am Ende der Erzählung Gestalt annimmt und Leben gewiinnt. Es wird nicht klar, ob es sich bei diesem "Pan ohne Flöte" um einen Landstreicher handelt oder um ein Tier, bzw. eine Pflanze. Er ist ein Teil der Natur, wie sie im Verlauf der Beschreibuns entstanden ist.

Ein weiteres Beispiel für diese Raumfiktion, bei der aus Zeichen ein völlig neuer Raum geschaffen wird, kann auch in Schulz' Gestaltung des imaginären Himmelsraums, eines stets wiederkehrenden Raummotivs, gesehen werden. Horizont, Wolken und Licht als Begriffe zur Bezeichnung wesensmäBig grenzenloser Gegebenheiten und nicht einzelner, raumfüllender Gegenstände sind für diese Art der Raumfiktion besonders geeignet.

Die Novelle "Emeryt" zeigt das Autoren-Ich in der Situation eines alten Mannes, der nach seiner Pensionierung nochmals in die Schule eintritt und sich wieder in die Welt versetzt, die die Situation des Schuljungen bildet: "Unter den Bänken herrschte tiefes Schweigen. Wir tauchten kichemd hinab, wanderten auf allen Vieren, beschnupperten uns wie die Tiere und führten in der Dunkelheit flüsternd die üblichen Transaktionen durch. Niemals werde ich diese seligen vormorgendichen Stunden vergessen, während es hinter den Scheiben langsam hell wurde." (S. 336)

In dieser friedlichen und glücklichen Situation bricht der Sturm los, ebenfalls ein Leitmotiv im Werk Schulz'. Der Sturm ist zunächst ein Naturereignis, wie es mit dem Herbst ganz selbstverständlich verbunden ist. Diesmal werden nicht seine Auswirkungen 
auf der Erde, in der Stadt, also in Bereich der raumfüllenden Gregenstände beobachtet, wie dies im Kapitel "Sturmwind" aus den "Zimtläden" der Fall ist. Diesmal ist sein Wirkungsbereich der Himmelsraum, welcher zuerst mit allen Erseheinungen und Verändierungen beschrieben, dann aber zu einem eigenständigen und wirkungsvollen Raum wird, welcher die Situation des Pensionisten prägt und inn in seinen Bereich hineinzieht. Es ist von einem ganz bestimmten Tag die Rede, an welchem diese Umgestaltung des Raumes stattfindet. "An diesem Tag wurde der Himmel schon frühzeitig gelb und spät. Er war auf diesem Hintergrund in trübgrauen Linien imaginärer Landschaften, großer und nebliger Einöden modelliert, die sich perspektivisch als immer kleiner werdende Kulissen von Hügeln und Falten entfernten, ineinander verschwammen und erst weit im Osten zerbröckelten, wo er sich plötzlich wie der wallende Rand eines davonfliegenden Vorhangs losriB und einen weiteren Plan, einen tieferen Himmel, eine Lücke von erschreckender Blässe zeigte - das blasse und bestürzte licht der fernsten Ferne, farblos und wasserklar, mit dem wie in endgültiger Verblüffung dieser Horizont endete und sich schloB. Wie auf den Stichen Rembrandts konnte man während dieser Tage unter diesem Streifen helle mikroskopisch deutliche Gebiete sehen, welche sich - übrigens nie gesehen - jetzt hinter diesem Horizont erhoben, unter dieser hellen Spalte des Himmels, mit grell blassem und panischem Licht überflutet, wie aus einer anderen Epoche und einer anderen Zeit emporgetaucht, wie ein dem sehnsüchtigen Volk nur einen Augenblick lang gezeigtes gelobtes Land." (S. 336 f.)

Das Verschwinden der konkreten Situation eines Beobachters, der beim Gang in die Schule den Blick vorüberge aend zum Himmel lenkt und dann seine Eindrücke schildert, kommt an der gegensätzlichen zeitlichen Bestimmung zum Ausdruck. "An diesem Tag" heiBt es zuerst, einige Sätze später aber "während dieser Tage"; schon am Morgen wird der Himmel "gelb und spät", d.h. er trägt die Anzeichen der Abenddämmerung. Die ständigen Bewegungen und Formveränderungen der Wolken werden durch den Versuch, eine Bewegungskomponente präzis zu erfassen, verlangsamt. Hier fängt die Umgestaltung an. Die erlebnismäßigen Situationskomponenten werden mit Zeichen versehen. Da sich das Wahrnehmungsfeld in stän- 
diger Veränderung befindet, wird eine bestimmte Phase, ein Augenblick dieser Bewegung festgehalten. Die Zeichen, die dieser momentanen Situation zugeordnet werden, entstamen einem anderen Bereich, in welchem nicht diese schnellen Veränderungen stattfinden und die deshalb leichter zu fixieren sind. Es ist der Bereich des Bühneninventars: Kulissen, Vorhang, Plan, Perspektive, Bühnenbild, Hügel und Falten.

Doch finden auch in diesem Wahrnehmungsfeld Bewegungen statt, die durch den thergang in einen anderen Bedeutungsbereich abermals fixiert werden: Ein Rembrandtstich mit einer Landschaftsdarstellung wird interpretiert. Im nächsten Abschnitt kommt zum Ausdruck, daß diese Symbole nicht mehr den Gegebenheiten des Wahrnehmungsumfeldes in einer bestimmten Situation des Betrachters zugeordnet sind, sondern durch ihre Kombination im Rahmen eines Sprechaktes eine eigenständige Situation bilden, deren Komponenten real erlebt werden. Diese bilden das Ausgangsfeld für die Bedeutungserfüllung weiterer Vorgänge, die mit den ursprünglichen Darstellungsobjekten, also Sturm oder Bühnenbild keinen Zusammenhang mehr haben. "In dieser hellen hiniaturlandschaft war mit wunderbarer Schärfe zu sehen, wie sich auf wellig gewundener Bahn ein in solcher Ferne kaum sichtbarer Eisenbahnzug bewegte, der sich zu einem Streifchen silberweißen Rauches aufplusterte und in helle Nichtigkeit zerrann." (S. 337 f.) 
9. Das Zeigwort als Orientierungssignal für die Figur und zeitliche Desorientierung des Lesers in Andrzejewskis "Bramy raju"

Die Genese des symbolischen Raums im Roman "Bramy raju" von Jerzy Andrzejewski 1, geschrieben 1959, kann nur auf der Grundlage der Zeitgestaltung, nämlich der vollkommenen Auflösung der chronologischen Proportionen und der Manifestation einer werkimmanenten Sukzession untersucht werden. Nur von der strukturellen Relevanz dieser Sukzession her kann die Konstruktion einer Welt und die darin wirksame Symbolik beleuchtet werden. Welche Sukzessionen können in diesem Werk, welches auf den ersten Blick eine amorphe Ansammlung von Zeitabschnitten bietet, festgestellt und voneinander abgehoben werden?

Da ist zunächst die historische Zeit, welche den Abschnitt von 1204 bis 1213 umfaßt. Im Romanverlauf tauchen von den historischen Fakten, die im Motto angedeutet werden, nur sehr wenige auf, und diese in sehr veränderter Form. Ein Hirt verkündet eine göttliche Botschaft und löst damit den Kinderkreuzzug aus. Der historische Schauplatz, also Frankreich, ist nur auf Grund einiger Ortsnamen zu identifizieren. Der totale MiBerfolg des Zuges liegt außerhalb des zeitlichen Rahmens des Romangeschehens. Der Grund für den Kreuzzug, der im Motto als "religiöses Traumdenken" bezeichnet wird, ist vollkommen umgestaltet. Die illusioristische Natur dieses Traumes bildet die zentrale Wirklichkeit ces Romans; man kann sie jedoch nicht mehr als religiös bezeichren.

Ife Folge der entscheidenden, schicksalsschweren Ereignisse konstituiert sich in einer Reihe von Nächten, deren chronologisches Eintereinander und erlebnismäßige Einmaligkeit im Zuge der Entlaltung des Romangeschehens nicht eingehalten sind. Es handelt sich un die Nacht der Zerstörung von Byzanz, die Nacht der Hochseit Agnieszkas und zugleich des schicksalhaften Zusammentreffens cer Piguren Ludwik und Jakub sowie Aleksy und Blanka. In dieser racht, deren genauer Verlauf schwer zu rekonstruieren ist, da sie nur im Spiegel der Erinnerung der fünf Kinder von Cloyes er-

- J. Andrzejewski: Bramy raju, Warszawa 1963. 
scheint, stirbt der Graf Ludwik eines mysteriösen Todes. Drei Tage später ist die Nacht der Offenbarung und des Aufbruchs, fünf Wochen später die letzte Nacht der allgemeinen Beichtzeit, von der es heißt: "Und sie gingen die ganze Nacht." (S. 111)

Genauso gut kann man den im Romanverlauf erfaßten Zeitabschnitt als den letzten der drei Beichttage ansetzen, welcher vom ersten bis zum letzten Satz reicht und in Form der fünf Beichten den BewuBtseins- und Erlebnisinhalt der Hauptfiguren aufrollt, unterbrochen nur von einigen Landschaftsbildem und den Gedanken des Mönches. Wesensmerkmal dieser Folge ist, daB alle Glieder den gleichen Sachverhalt betreffen und dieser nur in der $\mathrm{Zu}-$ sammenschau aller Glieder vollständig erfast werden kann. Dabei wird einerseits bei keiner Beichte auf den Inhalt der vorangehenden zurückgegriffen als auf etwas, was der Leser schon weiB, so daß sich im Lesevorgang eine Reihe von Wiederholungen ergibt. Andererseits ist der Inhalt keiner Beichte aus sich selbst heraus ohne die anderen verständlich. Zur Bedeutungserfüllung einer Beichte sind nicht nur die anderen als Situationskomponenten vonnöten, sondern auch die synsemantischen Umfelder der Zentralbegriffe, die in einer unkonventionellen und eigenwilligen Weise umstrukturiert sind und nur dadurch eine Bedeutungspräzision erfahren, daß sie von allen Figuren in der gleichen Bedeutung und für die gleichen Sachverhalte verwendet werden. Das Hintereinander der Fakten, wie es in einer solchen Beichte in Erscheinung tritt, ist bestimmt durch Assoziation und Erlebnisfolge des betreffenden Kindes und nicht durch eine Reihenfolge, wie sie für die Erfassung ihrer zeitlichen Position am günstigsten wäre.

Vom Inhalt des Romans, also vom Verlauf des Kreuzzuges her, ist zu bemerken, daB die Folge: Offenbarungsidee - Aufbruch - Weg Ziel, im Erzählvorgang eine Umkehrung erfährt. Das Ziel, das Grab Christi in Jerusalem, tritt als erstes in Erscheinung, und 2war in zweifacher Ausführung: als goldenes Tor in der $V_{i s i o n}$ der Kinder, und als Wïste mit den letzten zwei überlebenden Knaben im Traum des Mönches. Der Gang des Zuges durch die wechselnde Szenerie der Landschaft findet in verschiedenen Bildern seine Realisation, die wahren Motive der Kinder sind erst an SchluB 
der letzten Beichte klar zu erkennen.

Die Schilderung der Bewegung des Zuges ist beschränkt auf einige Hinweise, die sich über den ganzen Erzählvorgang verteilen. Dabei wird nicht eine marschierende Kinderschar beschrieben, sonderm das eintönige Geräusch der Schritte und die Details der mitgeführten Requisiten, das langsame Schwanken der Fahnen, Baldachine und Kreuze über den Köpfen der Kinder, ab und zu ein Glokkenton. Doch diese Einzelheiten verstärken nur die Unaufhaltsamkeit und Eintönigkeit dieser Bewegung, die keinen Einschnitt und keine Geschwindigkeit kennt. Der genaue Verlauf dieser Bewegung kann nur an einer Stelle bis in alle Phasen hinein verfolgt werden, nämlich da, wo sie beim dramatischen Höhepunkt des Geschehens für einen Augenblick zum Stillstand kommt. Der Mönch bleibt mit erhobenen Händen stehen, um den Zug aufzuhalten.

" ... mein Sohn - sagte der alte Mann und hielt in der Mitte des Weges an, er stand in der dichter werdenden Dämmerung riesig und schwer in seinem groben, braunen Habit, und als er sich mit dem Gesicht zu den Gehenden umwandte, hielten die ersten an, dann die, welche nach ihnen kamen, nach einer Weile war diese Beruhigung bis zu den letzten im Zug durchgedmungen, die jetzt schon fast unsichtbar waren, es wurde still ..." (S. 108)

Nach einem Augenblick des Erstarrens wird der Mönch von der Wucht des sich wieder in Bewegung setzenden Zuges überrannt und in den Boden gestampft.

Bei den bisher angeführten Sukzessionen handelt es sich um Einheiten, welche den Zeitablauf zergliedern, dabei aber immer von einer Linie ausgehen, deren Vorhandensein erst die Grundlage für eine zeitliche Anordnung und somit für eine Zeitgestalt bietet. Dabei konnte aber keine klar umrissene Anordnung festgestellt werden, da die verschiedenen Tatbestände, durch deren Folge sich eine Linie konstituieren könnte, durch tberlagerung und die Auflösung der kausalen Kette sich bei dem Bemühen um eine genaue zeitliche Lokalisierung im Romanzusammenhang nicht in einer eindeutigen Position festlegen lassen. Der Autor arbeitet der Orientierung des Lesers entgegen, indem er völlig verschiedene Sachverhalte übergangslos, d.h. im syntaktischen Zusammenhang formal 
nicht voneinander abgehoben, nebeneinanderstellt.

Durch die anakoluthe Konstruktion erreicht er, daß der Bewußtseinsinhalt und das Wahmehmungsumfeld zu einem einzigen Feld zusammenfließen: "... nur er blieb in seiner Hütte zurück, aber als es eines Abends geschah, daB er herauskam, der Schatten, der sie ständig mit seinem Schweigen begleitet hatte, da sie unverändert mit niedergeschlagenen Augen ging, der Schatten, der gleich inr gefangen war in einem Netz, welches aus dem Schatten des Dickichts und dem Glanz der unsichtbaren Sonne gebildet war, füllte sich plötzlich mit einer fast plastischen Körperlichkeit: er ist klein und nur wenig größer als ich ..." (S. 17) Daß es sich hier um ein Nebeneinander von Wahrmehmungsgegenständen und BewuBtseinsinhalten handelt, wird dem Leser erst klar, wenn er diese Zeilen mehrmals liest. Weder die Folge des Erinnerungsaktes noch die Folge des Wahrnehmungserlebnisses der Figur bilden eine Zeitlinie, anhand welcher die Position der Tatbestände eindeutig festgelegt werden kann. Es fehlen die dazu nötigen zeitlichen Orientierungssignale, welche die Einschnitte bezeichnen, sie einer bestimmten Zeitlinie zuordnen und den Wechsel von einer Linie zur anderen markieren.

Dafür ist eine andere Art von Einschnitten zu beobachten, die vom Bühnen- und Szenenwechsel unabhängig ist und anschaulich geschlossene Handlungseinheiten anscheinend willkürlich zerteilt. Es handelt sich dabei um immer wieder in den Erzählstrom eingeflochtene Formeln, die einen Punkt auf einer Iinie markieren. Diese Orientierungssignale dienen aber nicht dem Verständnis des Lesers, sondern tauchen im Zusammenhang mit dem Bemühen der Figur auf, Vergangenes zu rekonstruieren. Daß der Autor dabei nicht die Orientierung des Lesers anstrebt, zeigt sich in der ständigen Wiederholung dieser Formeln, welche die Klarheit der angedeuteten zeitlichen Relationen wieder zunichte macht. Es handelt sich um die Orientierungssignale vom Typ: "Jetzt herrscht noch Dunkelheit, aber in einer Weile wird es dämmern" (S. 10); "... immer habe ich vor dem Einschlafen ein Gebet gesprochen, jetzt aber, da ich Cloyes verlassen habe ..." (S. 13); "... ein Weilchen schwieg er, dann sagte er ..." (S. 24). 
10. Das Prinzip der revertierten Metaphorik zur Umstrukturierung der Sphären des Dinglichen und des Begrifflichen in Andrzejewskis "Bramy raju"

Wenn einerseits die Genese des werkimmanenten Raumes an die Sukzessivität des Erzählvorgangs gebunden ist, andererseits mit jedem Sprechakt auch die Möglichkeit der Genese einer räumlichen Ordnung gegeben ist, so ist es nach Freilegung der verschiedenen Sukzessionen schwierig, für den Roman "Bramy raju" einen greifbaren Anfang der räumlichen Darstellung zu finden. Zumindest in Schriftbild und Satzbau läBt sich nicht eindeutig eine erste Erzähleinheit finden, ein Anfangsglied, welches dem Sprechakt Gestalt und Geschlossenheit verleiht. Auf der zweiten Seite finden sich einige Sätze, die eine Landschaft darstellen. Ist diese Landschaft ein Schauplatz des Romangeschehens, so ist zu fragen, in welchem Maße dieser Raum für die Bedeutungserfüllung der Beichten relevant wird.

"... die ferne Sonne glomm gleichgültig über den Räumen von Schatten, Feuchte und Stille, mächtiger als ihr ferner Glanz war die Dunkelheit der gewaltigen Stämme und Wurzeln, Blätter und Äste, in der Morgendämmerung, da das Licht, noch scheu und brüchig, langsam über die Flächen von Grün und Schweigen aufstieg, schrien im Dickicht des Urwalds frühe Vögel, auch als die Abenddämmerung fiel, schrien sie, und in den Nächten, wenn sie gingen, um die Zeit der Beichte nicht zu unterbrechen, in den Nächten also, die angefüllt waren vom Rascheln der Tausende nackter Füße, drang zu inm das klägliche Rufen der Käuzchen, in der Finsternis schwankten lautlos die schwarzen Kreuze, Fahnen und Baldachine ..." (S. 8)

In diesem Abschnitt sind verschiedene Möglichkeiten der Schaffung eines Raumes angelegt. Von einem Standpunkt aus, welcher diesen dbschnitt aus seinem Zusammenhang herauslöst, können folgende Sechniken der sprachlichen Raumbehandlung festgestellt werden:

"Die ferne Sonne glomm gleichgültig ..."; der Autor verleiht einem Element der Landschaft eine Aktivität, eine eigentümliche Form des Lebens, ohne es zu personifizieren. Die Position der Sonne findet gesonderte Erwähnung, durch den räumlichen Abstand 
wird gleichzeitig die Art des Verhältnisses der betroffenen Gegenstände zueinander charakterisiert.

"Sie brannte über den Räumen von Schatten, Feuchtigkeit und Stille, von Grïn und Schweigen." Die zusammenfassenden Abstrakta fungieren nicht als Zeichen für einen Komplex, da sich in diesem keine weitere Untergliederung feststellen läBt, sondern als Zeichen für homogene Bereiche. Es handelt sich um das bemußte Sehen in den Kategorien einer räumlichen Ordnung. Das Verschwinden des Details kommt in den Worten: "die Dunkelheit der gewaltigen Stämme und Wurzeln, Blätter und Aste" zum Ausdruck. Die Dunkelheit wirkt räumlich, die Körper verlieren ihre Grenzen und werden nur im Zusammenhang als Raumteil gesehen, dessen gewaltige Dimensionen noch durch Kontrastwirkungen verstärkt werden, nämlich das klägliche Rufen der Käuzchen. Auch in diesen Raumteilen ist das Prinzip der lebendigen, kräftemäBigen Auseinandersetzung wirksam; die Dunkelheit ist stärker als das Licht, dieses ist noch brüchig und scheu. Licht und Dunkelheit deuten ein antithetisches Prinzip an, welches das Wortmaterial des ganzen Romans durchzieht. Diese Antithese führt im Zusammenhang mit dem Prinzip der lebendigen Auseinandersetzung $z u$ einer Gegenüberstellung von Himmelsraum und Erdraum und damit zur Entstehung einer Vertikalachse. Die Nacht ist voll vom Geräusch der Tausende nackter Beine; damit wird einerseits der Raum der Nacht mit etwas Unkörperlichem, mit Geräuschen angefüllt, wodurch diese eine plastische Gegenständlichkeit erhalten. Die Menschen treten andererseits nur durch Geräusche in Erscheinung, die einzelne Figur nur in der Masse, wodurch sie der Tendenz der Entindividualisierung und Verleblosung unterliegt.

Die Analyse könnte sich des Vorwurfs der tberinterpretation nicht erwehren, wenn sie nicht eine Reihe von Ergebnissen der Gesamtbetrachtung vorwegnehmen wïde. Von dieser Warte aus zeigt es sich, daB jedes einzelne dieser Merkmale, wie sie bei diesem ersten Auftauchen der Landschaft festgestellt wurden, sich durch den ganzen Roman hindurchzieht und dab nicht nur die einzelnen Landschaftsbilder davon betroffen werden, sondern der gesamte Romanverlauf von ihnen beeinfluBt, ja sogar gelenkt wird. So bedeuten 
auch die weiteren Landschaftsbilder nicht jeweils nur einen kurvenförmigen Anstieg der Relevanz räumlicher Ordnungsfaktoren, sondern die Herausbildung eines ganz unkonventionellen, eigenständigen Situationsfeldes, in welchem allein die Zentralbegriffe der Beichten ihre spezifische Bedeutung erfüllen. Die Konsequenz, mit welcher diese Darstellungstechniken weiterhin angewendet werden, ist für den Aufbau des Umfeldes wichtiger als die Weiterentwicklung der Naturereignisse. Diese veranschaulichen das Fortschreiten der Zeit, die Bewegung des Zuges, welche durch die homogenen Geräusche des Marschierens nicht zum Ausdruck kommt. Die konsequente Anwendung dieser Darstellungstechniken resultiert in der Aufrechterhaltung einer bestimmten räumlichen Wirkung und somit in der Kontinuität des situationsbedingten Umfeldes.

Als nächstes Landschaftsbild folgt die Vision des Mönches von der Hüste, in welcher er die letzten zwei Uberlebenden des Kreuzzuges sieht: "... sogleich begann der andere vor sich hinzugehen, und an seinen Bewegungen erkannte ich augenblicklich, daß er blind war, Gott - dachte ich - laß mich von diesem Traum erwachen, immer noch nicht konnte ich das Gesicht des blinden Knaben sehen, er ging einsam durch die tote und von der Sonne verbrannte Wüste, mit unbeholfenen Händen griff er in die Leere, als suche er für sie eine Stütze, und jener, der mit den ersterbenden Lippen schon den Wüstensand berührte, konnte noch sagen: es tagt, ich sehe die gewaltigen Mauern und Tore Jerusalems, die golden sind von einem Licht, von dem $i c h$ nicht weiB, woher es kommt, ob von den Mauem, Toren und Türmen oder aber von einem goldenen Schein, der über ihnen Luft und Himmel umfaßt, ... als dieser blinde, zarte und blondhaarige Knabe immer noch vor sich hinging und mit den Händen die leere Luft so annührte, als berührte er wirklich Mauern, sein Gesicht zu mir wandte ..." (S. $10 \mathrm{f}$.)

Noch radikaler ist der Raum hier reduziert auf die Fläche der Erde, noch intensiver wird so der Eindruck der Bewegung, die sich von Ausgangspunkt und Ziel befreit hat und, diesmal auch anschaulich sichtbar, nur noch aus sich selber lebt. Das Ziel der Bewegung des Zuges wird in einer dinglich greifbaren und körperlichen Form realisiert; es ist die anschauliche Realisation des Topos von der goldenen Pforte, die zur Ewigkeit, zum besseren Jenseits 
führt und als das Symbol für die Nähe des Heils hier in einer konkreten Ausführung vorliegt. Das Verschwimmen und Ineinandergehen von gegenständlicher und übertragener Sphäre wird durch die Tatsache verstärkt, daß dieses Bild einerseits ein Traum des Mönches, andererseits die Stadt Jerusalem in der Vision einer Figur im Augenblick des Todes ist. Dieselbe Vision vollzieht sich im selben Wortlaut eine Seite später noch einmal, und zwar unter so unklaren Umständen, $\alpha a \beta$ man auf Grund der Worte nicht entscheiden kann, um wessen Erlebnis es sich handelt: "... es dachte Jakub: ich hörte jedes Wort, welches er, in der Dunkelheit neben mir liegend, sprach, und zum erstenmal sah ich die gewaltigen Mauern und Tore Jerusalems ..." (s. 11) Erst am Ende des Romans wird der Leser erfahren, wann und wo dieses Gesicht stattgefunden und wer neben Jakub gelegen hat und welche Worte diese Person gesprochen hat. Dies ist aber keine Wiederaufnahme des Beginns, an welchen der Leser sich dann erinnert und wodurch sich die Aufbauform schlieBt, sondern eine formelhafte Wiederholung von Worten, welche überfigural in jeder Beichte auftaucht. Die Verselbständigung der Bewegung des Zuges kommt im dritten Landschaftsbild wieder zum Ausdruck; auch hier vermischt sich das den Sinnen der Figur sich Bietende mit einem visionären Ausblick auf die Zukunft, welcher die Zeit als einen Gegenstand mit lebendiger, dynamischer Natur sieht: "... er sah vor sich, als er jetzt an der Seite des alten Beichtvaters ging und nichts mehr vor ihm war, nur jener Waldpfad, der ihr gemeinsamer Weg war, ein vom Gras überwachsener, durch die gewaltigen Mauern des Urwalds dringender Pfad, erst jetzt, als er zum erstenmal nach dem Auszug aus Clojes an der Spitze des Zuges ging und niemand ihm den Weg, den sie zu bewältigen hatten, verstellte, wurde er sich der Vielzahl von Tagen und Nächten und ihres gleichförmigen Ablaufs bewußt, und sogleich, als er das dachte, begriff er, daB die Tage und die Nächte, die ihren endlosen Weg abmessen werden, keine geduldige Gleichgültigkeit mitbringen werden, sondern in der Hitze, wenn der Himmel über ihnen brennt und sie jeglichen Schattenstreifens beraubt sein werden, in der schweren Hitze, in den Stürmen und den über den entblöBten Räumen wild strudelnden Regengüssen, in den hartnäckigen Regenfällen und in all dem 
anderen, was die Erde und der Himmel Tas und Nacht mit sich bringen können, werden diese Tage und Nächte, deren Vielzahl sich gar nicht erfassen läBt, sie verfolgen und quälen, nur selten werden sie die Kinder mit wohlwollenden Gaben beschenken, um sie dann wieder, nach kurzen Stunden der Erleichtemung und der Sicherheit, aufs neue in den Abgrund der Elemente zu stoßen ..." (S. 24 f.)

Hier ist nicht ein bestimmtes, hic et nunc stattfindendes Naturereignis beschrieben und die Folge von Ereignissen in ihrer realiter stattfindenden Form; sondern gerade die Zusammenfassung aller möglichen Ereignisse zu einem einheitlichen Eindruck bewirkt das lebendige, personale Prinzip in der Natur und die Vergegenständlichung der Zeit. Diese wird zu einem dynamischen Gebilde, dem, ohne daß eine Personifizierung vorläge, auch Gefühlsregungen der größten Dimensionen zukommen. Hier fangen die Gewichte an, sich zu verschieben. Die Sphären des Dinglichen und des Lebenden, welche literarisch in metaphorischer und somit verdeutlichender oder veranschaulichender Weise sich vermischen, so daß im sprachlichen Darstellungsvorgang ein Element der einen Sphäre in die andere übergeht, gewinnen in der neuen Umgebung Gestalt und Leben und greifen aktiv in den Romanverlauf ein. Dieser ProzeB geht so weit, daß am Ende des Romans der Ubergang von einer Sphäre in die andere vollendet ist.

In der nächsten Landschaftsbeschreibung wird das raumimmanente Leben zu einem Höhepunkt geführt: die Spannung entlädt sich in einem Gewitter. Das aktive Bewegungsmoment der Elemente, welches mit seinen Dimensionen den erlebenden Menschen überwältigt, ist vorherrschend, so daB dessen Erlebnis in gleichen Intensitätsgraden verläuft: Panik und Entsetzen. "... Und als sie in Panik ihre Augen erhob, sah sie vor sich: den Himmel, der mit seiner ungeheuren Größe den ganzen Horizont umschloß, dunkle und schwere Wolken sammelten sich dort, weiter unten, plötzlich von den ungeheuren Mauern des Urwalds befreit, breitete sich die weite Niederung aus, flach und vom drohenden Schein der schweren Wolken gesättigt, sie erblickte einen kurzen, eiligen Blitz, der das unbewegliche Dickicht der Wolken zerriB, unten sah sie grüne Teiche, einsame Bäume, es umfing sie eine Luft, die leichter und 
weiter war als die Luft, die sie bisher geatmet hatte, der Weg fiel nach unten $a b$, wo inmitten der flachen Niederung sich in unbeweglichem Grün grüne Teiche öffneten und wo aus der grauen und unbeweglichen Erde einsame Bäume wuchsen, von neuem brach ein ungeduldiger Blitz durch die Wolkenmassen, schwarze Krähen lösten sich von den letzten Bäumen an Rande des Urwaldes und flogen in schwarzem Schwarm niedrig über die Erde, sie flogen zu den grünen Teichen, ein ferner Donner wälzte sich irgendwo sehr weit weg ..." (S. 44)

Den Schlüssel zum Wesen dieses Bildes bietet das Zeigwort "plötzlich"; seine Funktionen an dieser Stelle, da die weite Ebene, von den Mauern des Urwaldes befreit, sich ausbreitet, sind sowohl deiktisch als auch symbolisch im Sinne der Differenzierung Bühlers, indem sie nicht nur hinweisen auf einen bestimmten ort in einem begrenzten Bereich, sondern daß sie diesen Ort auch nennen, daß also mit der Aktualisierung dieses Signals im konkreten Sprechakt die Möglichkeit einer Situationserweiterung um eine zusätzliche Komponente gegeben ist. Anzeichen für die Ausnutzung dieser Nöglichkeit sind besonders auf dem Sektor der Zeitgestalt zu suchen. Denn hier kann durch Häufung und regelmäßige Wiederholung sowohl eine Situation oder ein komplexes Objekt gleich welcher Art dargestellt, als auch eine Sukzession eigenständiger Art aufgebaut werden.

Im Fall der Figur Blanka, die an der Spitze des Zuges den Urwald verläßt und eine weite Ebene vor sich sieht, zeigt das Wort "plötzlich" einerseits auf einen Punkt der Zeitlinie hin, welche von der Kette ihrer Wahrnehmungserlebnisse gebildet wird. Andererseits wird die momentane Situation, in welcher allein das Zeigwort seine Bedeutung erhält, genannt und dem Leser vor Augen geführt. Die Komponenten dieser Situation werden nicht von Wahrnehmungsgegenständen allein gebildet, vielmehr werden diese nur durch das ihnen innewohnende Bewegungsmoment für die Bedeutungserfüllung des Zeigworts "plötzlich" relevant. Wo in diesem Wahrnehmungsraum tatsächlich etwas Unbewegtes auftaucht, wird es nur im Zusammenhang und als Kontrast zu einer Bewegung genannt. Die 
unbeweglichen Teiche "tun sich auf", aus der unbeweglichen Erde "wachsen" einsame Bäume. Die auktorale Fähigkeit zu intensivem Erleben einer Räumlichkeit auch da, wo kein realer und den Sinnen zugänglicher Raum gegeben ist, lenkt hier den Erzählverlauf. Sie steuert die Fiktion und läBt den Szenenwechsel vom Waldweg zur Ebene zu einem dramatischen Geschehen werden, welches nun seinerseits wieder die Handlung der Menschen mitreibt und ihren Verlauf bestimmt.

Seinen Ausdruck findet dieses gegenstandsunabhängige Raumerlebnis im zentralen Thema, der unerfülten Liebe, welche von den Figuren nicht nur räumlich erlebt wird, sondern als räumliche Kraft das Handeln bestimmt und letzten Endes den Aufbruch nach Jemusalem verursacht. Durch das "plötzlich" wird nicht nur ein Punkt auf der Zeitlinie des erzählten Vorgangs bezeichnet, es ruft die emotionalen Situationskomponenten der Figur im Erlebnisbereich des Lesers wach, wodurch die Darstellung momentaner, gleichzeitiger Vorgänge möglich wird und in Form von dramatischen Höhepunkten die Situation des Iesers mit der der Figur zusammenfällt. In der Beichte der Blanka sieht das so aus: "... plötzlich, wie aus langwährender Starrheit geboren, riB sich der Wind 108 , eine gelbe Staubwolke stob über die flache Niederung, ich habe gelogen - schrie Blanka - alles habe ich gelogenl, sogleich, ohne ein Wort zu sagen, erhob der alte Mann die Hand und schlug uiber ihrem Kopf das Zeichen des Kreuzes, Aleksy Melissen atmete in diesem Moment vor Erleichterung auf ..." (S. 47 f.) Die Gleichzeitigkeit all dieser Vorgänge, die durch die einheitliche Aktionsart der Verben noch unterstrichen wird, ist nur möglich auf Grund der räumlichen Situation, durch deren momentanen Charakter die Situationsentbindbarkeit der Darstellung realisiert ist. Die am Raum festgestellten Wesenszüge kommen nicht nur in den Landschaftsbildern zum Ausdruck und verhelfen diesen zu einem eigenständigen dramatischen ProzeB. Die Techniken, räumliche Situationen als Darstellungsfeld zu verwenden, gelangen auch an geistigen und mentalen Tatbeständen zur Realisation, durchziehen das ganze Verk und lenken den Verlauf des Geschehens.

Dem Bestreben, unkörperlıch zu sehen, die Grenzen der Körper über diese hinaus $z u$ verlängern und zu verbindenden, den Raum einteilen- 
den und gestaltenden Iinien bzw. Kräften zu machen, folgt das Auswahlprinzip, welches nur noch kollektiv, unkörperlich und in letzter Konsequenz überindividuell sieht. An die Stelle der verlorengegangenen Körper und Gegenstände treten Begriffe und Symbole und übernehmen deren raumaufbauende Funktionen, obwohl sie keine Körper und Gegenstände, keine Objekte sind, sondern das Verhältnis von Subjekt und Objekt bzw. die Art dieses Verhältnisses betreffen. Darin kommt das zentrale Thema der ideologischen Verirrung zum Ausdruck. Der Charakter des Raumes bietet erst die Grundlage und die Voraussetzung für diese Umgestaltung.

An die Stelle der Körper treten zunächst Sammelbegriffe, Abstrakta, Pluralia: "... die Hütte, die er sich am Rande des Urwalds errichtet hatte, erhob sich über den Wiesen, und wenn er davorstand, konnte er die ganze Weide überblicken, oft wünschte ich, ich könnte unsere Weide mit seinen Augen erblicken, mit Augen, die rein sind und für deren Reinheit ich kein wahres Gleichnis finden kann, sie sind rein; als sich die ersten Schatten auf die smaragdene Niederung zu legen begannen und der Himmel sich mit Violett und Stille gesättigt hatte, in den Wiesen zirpten die Grillen und die Vögel riefen einander vor dem Schlaf im Eichendickicht ..." (S. 16) Wiese, Weide, Niederung und Eichendickicht sind räumliche Gegebenheiten ohne feste Begrenzung und Kontur. Die Vögel und die Grillen tauchen nur in der Mehrzahl auf und sind nicht fest lokalisierbar. Die Abstrakta Violett und Stille werden schon mehr in den Bereich des Körperlichen hineinverlagert durch den Vorgang des Sättigens, des Aufsaugens, der sich mit ihnen verbindet. Vollkommen zum Gegenstand ist die Reinheit geworden, die als Begriff in keiner Weise fest umrissen und definiert ist, aber lokalisiert und ausgetauscht werden kann wie ein greifbarer Gegenstand.Alle abstrakten Begriffe sind mit diesem Merkmal verbunden; dafür einige Beispiele: ".. und so habe ich alle Hoffnungen und Sehnsüchte in diese Kinder gelegt, die zu einem Ziel streben, welches sie und mich und alle Menschen auf Erden übersteigt, Gott, sei bei diesen unschuldigen Kindern, ich, dem keine Sünde fremd ist und der ich bis zum letzten Atemzug alle Verirrungen kenne, ... ich kenne ebenso gut den Boden 
dunkler Abgründe wie die imaginären Schimmer der Sehnsucht ..." (S. 9) "... seine Worte kreisen ohne UnterlaB in uns und außer uns ... damals verstanden wir sie noch weniger als jetzt, da diese Worte sich in den Weg verwandelt haben, der sich vor uns herzieht, und in ein Ziel, das wir uns nicht vorstellen können, aber welches unser Ziel wurde, und welches irgendwo im unbekannten Land und unter einem unbekannten Himmel vor unseren Augen schärfere Gestalt annimmt in Form von Toren und Mauern einer unbekannten Stadt ..." (S. 29 f.) "... und wenn man sagen kann, daB man die unbekannte Zukunft vor sich sieht, so habe ich sie damals gesehen, und obwohl in mir nur Finsternis und Verzweiflung waren, kein Glaube und keine Hoffnung, nur Finsternis und Verzweiflung, denn in dieser Stunde war meine Liebe zu Maud nur Finsternis und Verzweiflung ..." (S. 31 f.) Die Situation, in der diese Zeichen ihre Bedeutung erfüllen können, lst nicht die der Figur, die thren inneren Zustand klären und durch Bilder verdeutlichen will, sondern eine reale Spannung zwischen antithetischen Begriffen, die sich in einer räumlichen Bewegung entlädt. Sie wird durch die ständige, konsequente Wiederholung und die Tatsache, daB sie von allen Figuren in gleicher Weise erlebt und realisiert wird, zum konstituierenden Moment im Aufbau einer räumlichen Ordnung. Dazu kommt, daB hinter keinem dieser Begriffe eine fest umrissene Vorstellung steht, sondern gerade die Unklarheit über die eigentlich übertragene Natur der Begriffe diese erst befähigt, als räumliche Kräfte zu wirken.

Die Verkörperlichung ist eine Technik, die der Autor bei der Gestaltung seiner Welt anwendet. Es ist weiterhin eine Tätigkeit der Figuren auf Grund ihres ideologischen Wunschdenkens. Denn diese Wesenszüge werden nicht nur bei der Gestaltung des Schauplatzes, der Umwelt der Figuren relevant, sie wirken auch auf die Figur ein und bilden im GesamtprozeB der Auseinandersetzungen eine entscheidende Komponente. Dies wird besonders deutlich am Verhältnis der Einzelfigur zum Zug, zur Kinderschar. Obwohl sich die füf beichtenden Kinder als erste zu dieser Art von Geneinschaft zusammengeschlossen haben, sehen sie den Zug, wie er zur Zeit der Beichte vorliegt, stets als ein Kollektiv, als einen Gegenstand if Wahrmehmungsraum, so daB ihr Beobachtungsstandpunkt 
stets auBerhalb dieses Zuges liegt. "... und obwohl in Reichweite eines Armes niemand neben ihr war, fühlte sie, daB so, wie die Schatten des Urwaldes und der Widerschein der unsichtbaren Sonne das steife WeiB inres Kleides umwebten und umstrickten, ihr Körper von der unsichtbaren und schweigenden Menge umstrickt war, sie wuBte, daB dort hinter ihr, die sie an der Spitze des Zuges ging, zwischen den Schatten des Urwaldes und dem Widerschein der unsichtbaren Sonne schwarze Kreuze, Fahnen und bunte Baldachine schwankten und unter ihnen wie ein gewaltiger Atemzug der eng zusammengedrängte Haufe heller und dunkler Kinderköpfe dahinglitt ..." (S. 15) Die Kinder treten nur als Masse in Erscheinung, die Bewegungen sind nicht einzeln, sondern kollektiv; ein einziger Wille wird von diesem Wesen verkörpert: "... plötzlich drang zu ihr eine Bewegung aus dem ihr folgenden Zug, sie hörte etwas, das ihr wie ein Seufzer der Erleichterung oder der Vermunderung schien, gleichzeitig aus der Brust von Tausenden auggestoBen ..." (S. 43) Diese Betrachtung der Masse als Teil des Raumes ist nicht nur die Interpretation eines Autors, der mehr weiB als die beteiligten Figuren und aus dem überlegenen Urteil zu diesem Erlebnis gelangt. Die Interpretation dieser Massenerscheinung wird von der Figur selbst geleistet: "Das Bedürfnis nach Gewalt und Grausamkeit zerreiBt die Natur des Menschen, der Mensch läuft ihm davon, er läuft der Einsamkeit davon, er fürchtet sie und schämt sich ibrer, in der Herde dann ist er stark und rasend, er übt Gewalt aus und verbreitet Gewalt, er ist blind, er ist taub, aber er ist stark, weil er rasend ist ..." (S. 65)

Der Zug kommt dann so zustande, daB nicht eine einheitliche Kraft im einzelnen Kind wirkt und sie alle zusammentreibt, sondern eine Bewegung, die alles in ihren Sog hineinreiBt und der keines widerstehen kann. Ein Merkmal dieser Kraft ist die Ausschaltung der Sinnestätigkeiten in bestimmten Situationen. Die Kinder sind nicht an sich blind oder taub, sondern sie sehen nichts um sich herum als nur die anderen Kinder. Aleksy z.B., eine der Hauptfiguren, sieht diese Herde und den Wahnoinn, der sie vereint; er sieht sie wie einen Teil der Landschaft, er sieht ihre Blindbeit, und trotzdem schlieBt er sich ohne Zögern an: "Endlich erblickte ich sie, unter dem gewaltigen Himmel in solcher Vielzahl, das un- 
ten die ganze Ebene in langsamen Wellen zu fließen schien, Kreuze, Fahnen und Baldachine schwankten in der Sonne und erhoben sich über diese fließende Ebene, sie aber bewegten sich gleich langsam vorwärts, mit der unermeBlichen Menge von Köpfen eine Einheit bildend ..., ich sah die Gesichter von tausend Mädchen und Knaben, rot und verschwitzt, wit weit geöffneten Mündern, mit Mündern, welche sangen, aber die mir, der ich vorbeiritt, vorkamen, als seien sie vor einem diese zarten Körper in grauweißen Kleidern und groben Dorfkitteln lähmenden Erstaunen geöffnet, ... sie gingen zusammengedrängt, Gesicht an Gesicht, Arm an Arm, Körper an Körper, in eine gewaltige und blinde Herde gezwängt, blind, weil sie weder den Himmel noch die Erde sehen konnten, nur die Köpfe und Arme der vor ihnen Gehenden, sie gingen gedrängt, als schöpften sie aus der Gedrängtheit ihrer Körper die Kräfte für den weiteren unbekannten Weg ... (S. 80 f.)

Der innere Abstand, der aus dieser Beschreibung und Interpretation des Wahrgenommenen spricht, hindert die Figur jedoch nicht, sich diesem Zug ohne Zögern anzuschließen. Das Feld, welches durch die zentralen Begriffe des auktoralen Sprechaktes gebildet wird und dem Leser zum Verständnis und zur Orientierung dient, ist für die Figur zu einer räumlichen Situation geworden, in die sie sich hineinbegeben und in der sie sich bewegen kann.

Die Sphären des Räumlichen und des Unräumlichen erfahren eine grundsätzliche Unstrukturierung. Räumliche Elemente wie Teile der Landschaft und materielle Körper werden im Sprechakt des Autors durch Abstraktion, Zusammenfassung unter Begriffe sowie Dynamisierung ihrer konkreten Gegenständlichkeit beraubt. Sie sind nicht mehr Objekte von Wahrnehmung und Erlebniskraft, sondern greifen aktiv und bestimmend in die Sphäre des wahrnehmenden Menschen ein. Dadurch geraten geistige Sachverhalte und Begriffe auf die Objektseite, werden zu Dingen und plastischen Gegenständen, an welchen die räumlichen Kräfte zur Auswirkung komnen. Diese Umformung, welche zunächst am sprachlichen Material nachgewiesen wurde, wirkt nicht nur auf den Leser, welcher sich jit dem Sprachwerk konfrontiert sieht, sondern das Endergebnis lst eine autonome Realität, in welcher die Romanfiguren sich bewegen und von welcher ihr Leben geprägt wird. Am deutlichsten 
kommt das zum Ausdruck am Wort "Liebe"; diese wirkt im Romanverlauf nie als ein Motiv, eine Kraft, die die Handlungen der Figuren lenkt, sondern wird behandelt wie ein raumfüllender Gegenstand, der lokalisiert und begrenzt werden kann: "... nicht die Liese zu dir hieß mich zu deinem fermen Grabe gehen, sonderm eine andere Liebe ist in mir, eine Liebe, die alle meine Gedanken ausfüllt und in meinem ganzen Körper ist, in meinem Mund, meinen Händen und Augen, sie ist in mir, diese Liebe, als wäre ich sie selbst mit allem, was ich bin, die Liebe, die meine Gedanken und meinen Körper ausfüllt, hat mich geheißen, mein Elternhaus zu verlassen ..." (S. 14) Was hier auf den ersten Blick, an dieser einen Stelle wie ein Wortspiel oder eine Metapher aussieht, erweist sich im Romanverlauf nicht nur als eigenständige Kraft oder geschlossene, unabhängig vom spezifischen Ubertragungserlebnis wirksame Sphäre, sondern als die zentrale, werkimmanente Realität. Aus der Manipulation mit Begriffen wird die Manipulation der Realität, dies ist nur möglich auf der Grundlage der spezifisch auf die Bedeutungserfüllung dieser Begriffe zugeschnittenen Umfelder in Form eigenständiger räumlicher Situationen. Dazu dient die Herausbildung einer ganzen Reihe räumlicher Motive, die man zwar mit traditionellen Oberbegriffen bezeichnen kann, die aber in jedem Fall, dem spezifischen, werkimmanenten Bedeutungsfeld entsprechend, in neuartiger Ausführung realisiert sind. Es tauchen die Wotive Blindheit, Einsamkeit, Irrweg, Echo, Flucht und Grab auf. Die Einsamkeit ist aber nicht ausgeformt als die Isolation der Figur innerhalb eines begrenzten Ortes, sondern allein die genaue Ortsbestimmung ist schon die Abgrenzung gegenüber dem Ort, wo die andere Figur sich befindet und somit ausreichend für den Nachweis und das Erlebnis der Einsamkeit. Die ständige Angabe der Figurenposition vom Typ "nicht weiter als eine Armeslänge entfernt, vor, neben oder hinter einer anderen Figur" ist bereits die Realisation des Einsamkeitserlebnisses der betroffenen Figuren. Ebenso ist es mit der Blindheit; diese ist nicht die organisch bedingte Unfähigkeit zu visuellen Wahrnehmungsakten, sondern mit jedem figuralen Wahrnehmungsakt ist das Bewußtsein verbunden, das eigentliche Wesen der Dinge nicht erfassen zu können, durch den Gesichtssinn keinen Zugang zur Welt zu haben. Auch die Flucht ist auf diese Weise realisiert; sie ist 
nicht Bewegung weg von einem bestimmten Verfolger, von dem sie eine bestimmte Richtung erhält, sondern jede Bewegung der Figur ist mit den Erlebniskomponenten der Flucht verbunden.

Die Schematisierung der Positionssignale im deiktischen Feld bewirkt Verselbständigung der Stellen des Symbolfeldes. Die Partikel fungieren nicht als Zeighilfen, sondern als Symbole. Dies führt zur Genese eines räumlichen Leitmotives, welches mit seiner durchgehenden und konsequenten Realisation im Erzählvorgang eine Sukzession sui generis bildet: die gegenstandsunabhängige Positionsbeschreibung, die relativierende Lokalisierung sowohl zeitlicher als auch räumlicher Art. Dies reicht von der formelhaften Wiederkehr der Einschnitte vom Typ: ein Weilchen schwieg er, dann sagte er ... usw., bis zur Grundsituation der Figuren und somit dem eigentlichen Leitmotiv: Der Mensch reißt sich von seinem Ausgangspunkt, seiner Heimat los, bricht auf in die Fremde und kann das Ziel nicht erreichen. "... und als er, schon fremd und fern weiter vor sich hinging, mit Mühe die Erde mit den bloBen Füßen berührend, stand sie allein, schon losgerissen von jenem Menschen, aber noch nicht umfaBt von den Schatten, die allmählich zu lebenden Menschen wurden ..." (S. 22 f.) 
Der Raumbegriff und die damit verbundenen Termini erfüllen in der Wissenschaft zwei Funktionen. Sie kommen erstens dort zur Anwendung, wo ein Raum vorliegt und wo sein Zustandekommen, seine Merkmale und Grenzen erfaßt werden sollen. Sie dienen also der Analyse von Objekten, deren räumlicher Charakter außer Zweifel steht bzw. auf einem definierten Weg gewonnen wurde. Zum zweiten findet sich der Raumbegriff dort, wo ein bestimmter Realitätsbereich untersucht werden soll und der Raum als von der Wissenschaft konstruiertes Modell eingesetzt wird, welches der Schaffung von Grenzen, Einheiten und Schichten dient. Unter diesen beiden Aspekten, dem Raum als problematischem Untersuchungsgegenstand auf der einen Seite und dem Raum als analytischer Hilfsebene zur Untersuchung problematischer Gegenstände auf der anderen Seite, kann man jeden raumbezogenen Terminus betrachten.

Während nun für die Wissenschaft allgemein genau angebbar ist, ob ein solcher Terminus in der einen oder in der anderen Funktion benützt wird, ob als semantische Größe zur Bezeichnung räumlicher Gegebenheiten oder ob als Teil einer wissenschaftsinternen Konstruktion, ist eine solche Trennung für den erzählenden Sprachgebrauch nicht möglich.

Ein Beispiel mag illustrieren, wie in der literarischen Analyse beide Nöglichkeiten vorhanden sind, den Kaumbegriff fruchtbar einzusetzen, jeweils aber ganz verschiedene, einander widersprechende Ergebnisse erzielt werden. Die Beispiele stammen aus der Interpretation von Eichendorffs "Taugenichts".

H. Meyer 1 bezeichnet den Raum dieser Novelle als offen und charakterisiert ihn durch das Fehlen von Raumachsen: "Schall hat eminent raumbildende Funktion ... akustischer Tiefenraum ..., in welchem sich Ferne und Nähe, Höhe und Tiefe miteinander vermählen und verschmelzen ... Deshalb suggeriert der Schall eine Einerleiheit der unterschiedlichen Orte im Raum und übt er eine entgrenzend-vereinheitlichende Wirkung aus ... Denn diese Eestimmtheit (der Achse Wien-Rom) ist nur äußerlich-vordergründig und in ästhetischer Hinsicht nur scheinbar; in Wirklichkeit wird alles,

$1 \mathrm{H}$. Meyer: Raumgestaltung und Raumsymbolik, a.a.0., S. $627 \mathrm{f}$. 
was einer Verfestigung der Raumachsen nur entfernt ähnlich sähe, willentlich ferngehalten, weil sie der Glückseligkeit des freien Schweifens nur im Wege wäre." 1 Dadurch entsteht"allseitige Offenheit und das märchenhaft Utopische eines Raumes jenseits von fester Richtung und bestimmtem Lokal." Dieser Dimensionslosigkeit als Ergebnis der Analyse von Eichendorffs Raum steht das Ergebnis Alewyns gegenüber, der gerade in Gegliedertheit, Achse und Raumzone das Wesen der Landschaft dieses Werkes erblickt. "Ferne ... liegt durchaus in der Landschaft und nicht im Unendlichen, wie überhaupt die Kategorie des Unendlichen dem Lebensgefühl der empfindsamkeit oder der Philosophie des Idealismus entstammt und der eigentlichen Romantik durchaus fremd ist. Das sogenannte Unendliche, in seiner säkularisierten Form ein Euphemismus für das Nichts, kommt bei Eichendorff als Begriff kaum vor ... Eichendorffs Landschaft ist dagegen die endliche und geGliederte Landschaft." 2

"Wir werden bemerken, wie die zuerst zusammengeschrumpfte Landschaft sich ausdehnt, indem auf der Achse, die durch Bewegung dargestellt wird, Abstände eingetragen und damit Raumzonen abgesteckt werden, die den Zwischenraum ausfüllen zwischen dem Hier und jenem anderen Rande, an dem die Ferne liegt. Indem Landschaft so erfüllt wird, wird sie zugleich gedehnt, die Ferne wird, indem sie vermittelt wird, zugleich hinausgerückt. So entsteht Raumtiefe, und damit erhält der Raum seine wichtigste Dimension." 3 Wlan muß also die Wege genau von einander trennen, um solche therschneidungen und Doppeldeutigkeiten zu vermeiden, und sich stets darüber im klaren sein, ob man die räumliche Terminologie benützt, um Eigenschaften des Ërzählwerks zu charakterisieren, wie dieses sich etwa dem Raum gegenüber verhält, oder un, ausgehend von einem rorhandenen Raum, zu analysieren, wie dieser mit speziell sprachichen Mitteln und Techniken entstanden ist.

ine weitere Zweiteilung schließt sich an diese an: Die Frage rach künstlerischen Werten und ästhetischen Effekten des im Herk

1 H. Meyer, a.a.0., S. 208 f.

2 R. Alewyn, a.a.0., S. 57.

j R. Alewyn, a.a.0., S. 58 . 
vorgefundenen Raumes muB getrennt werden von der Frage nach dem technischen Aspekt. Dieses letztere Problem, welches von mir vorrangig behandelt wurde, kann man allgemein so formulieren: Wie muß eine sprachliche Gegebenheit von der Größe eines Romans mit hunderten von Seiten überhaupt beschaffen und organisiert sein, um noch als eine einheitliche Gegebenheit verstanden $z u$ werden und wirken zu können? Wie ist die Fülle von mit dem Erzählwerk verbundenen Fakten angeordnet, welche Erfordernisse stellt die speziell buchmäßige Speicherungsform? Die Sprache erlaubt freilich ausschließlich eine sukzessive bzw. sequentielle Anordnung des Materials. Daß daneben in der Sprache auch Ordnungsschemata simultan wirksamer, räumlicher Art vorliegen, düfte außer Zweifel stehen; mit dem Problem räumlicher Beziehungen im für die Sprache verantwortlichen Teil des Gehirns beschäftigt sich besonders die lieurolinguistik 1 .

Zielt man sogleich auf die künstlerischen Effekte des literarischen Raumes hin, so gerät man leicht in den Fehler, Art und Intensität rüumlicher Wirkungen in eine direkte, ursächliche Beziehung zur Adäquatheit raumbezogener Zeichen und Erzählphasen zu bringen und einen wirkungsvollen Raum dadurch einer genaueren Analyse zu unterziehen, daß man ihn möglichst genau rekonstmuiert. Am Beispiel des Romans "Quo vadis" von Sienkiewicz war zu sehen, daß die intensive Raumwirkung gerade durch die Ungenauigkeit der Raumdarstellung verursacht wird, so daß Adäquatheit der raumbezogenen Zeichen ein diaßstab der Analyse wäre, der dem Phänomen nicht gerecht wird.

Das semantische Problem kann zwar einen ersten Ausgangspunkt bilden, der noch von sprachwissenschaftlicher Art ist: Der Raum ist ein Gegenstand, der von Zeichen vertreten wird. Diese können mit semantischen rategorien charakterisiert werden. Sie können aus dem Erzählverlauf herausgenommen und isoliert betrachtet werden. Auch der Autor wählt, wenn man eine entsprechende Ebene ansetzt, isolierte Zeichen aus. Diese Untersuchungsebene habe ich durch den Begriff Adäquatheit abgegrenzt. Es geraten aber immer nur

$1 \mathrm{Vgl.A.R.} \mathrm{Lurija:} \mathrm{Problemy} \mathrm{i} \mathrm{fakty} \mathrm{nev} \mathrm{rolingvi} \mathrm{stiki,} \mathrm{in:} \mathrm{Teorija}$ rexevoj dejatel'nosti, Moskva 1968, S. 198-218, besonders $S$. $208 \mathrm{f}$. 
Zeichen ins Blickfeld, also zeitlich nicht ausgedehnte Größen. Die Notwendigkeit, zeitliche Einheiten zu finden, wird durch folgenden Gedanken motiviert:

Prinzipiell stehen für die Analyse sprachlicher Objekte drei Wege offen. Sie kann einsetzen, wenn das Sprechen bereits vorbei ist und das Produkt als strukturierte Menge von Zeichen vorliegt. Sie kann vor dem konkreten Sprechakt einsetzen und die Möglichkeiten und Grenzen abstecken, innerhalb derer zuküftige Sprechereignisse stattfinden und sich bewegen werden. Und sie kann das Sprechen während seines spezifischen Verlaufs, in statu nascendi beobachten, und dann werden die Entscheidungen wichtig, die steuernd wirken, und die Größen, die vom Sprecher als relevant empfunden werden, nicht vom sprachwissenschaftlichen biodell her gesehen relevant sind. Adäquat ist hier, was der Sprecher als angemessen empfindet, und nicht was die Semantik als adäquat bezeichnet. Ein anderes Beispiel: Ein Sprecher kann eine unvollständige und wenig geglückte iormulierung durch einen zusätzlichen Sprechakt ergänzen oder gar außer Kraft setzen. Solche logischen Inkonsequenzen sind keinesfalls seltene Ausnahmefälle. Die Analyse kann dann aufzeigen, wie solche "Umbrüche" von der Sprache bereits vorgesehen, aie Mittel zu inrer Realisierung im Sprachsystem enthalten sind und die Verbindung von heterogenen Sprechteilen zu einem geschlossenen Sprechakt möglich ist.

Die zuerst genannte Betrachtungsweise, die bisher den weitaus bevorzugtesten Platz einnahm, ist deshalb für das Erzählen unzureichend, weil ein Zeitraum "nach dem Sprechakt" beim Erzählen gar nicht vorhanden ist, da er ja per definitionem bereits wieder vom Erzähler okkupiert ist. Will man dennoch kleinere zeitliche Einheiten heraussegmentieren, so muß man diese genau absichern, um durch diese Isolierung nicht wesensmäBige Veränderungen zu verursachen.

Sucht man also beim Phänomen Erzählen nach zeitlichen Einheiten, so muß man sich dessen bewußt sein, daß diese nicht zeitlich veiter untergliedert werden können, indem man sich diese Einheit als aus Zeichen bestehend vorstellt, denn diese sind zeitlich ausdehnungslos. hian kann einem Zeichen einen zeitlichen Stellenwert zuschreiben, wenn man ihm entweder eine außersprachliche 
Zeitlinie zugrunde legt oder ihm eine vorgangsbildende Funktion zubilligt.

Ich vollziehe den Schritt zur zeitlich ausgedehnten Einheit in der auf das raumbezogene Erzählen konzentrierten Analyse nicht durch den Rückgriff auf psychische Vorgänge auf der Seite des Erzählers oder des Zuhörers, sondern durch folgende Konstruktion: Bemüht sich der Autor um adäquate Darstellung eines Gegenstandes oder Vorgangs, indem er semantisch optimale Zeichen auswählt, so ist er, gemäß dem Charakter des Erzählens als Folge von Sätzen, nicht von Zeichen, gezwungen, eine weitere, meist zahlenmäßig stärkere Zeichenmenge $z u$ den ausgewählten hinzuzufügen, die, gemessen am ursprünglichen Objekt, redundant sind. Für die Erzühlung als Kunstwerk schließe ich jedoch den Sachverhalt der Redundanz aus, da die Analyse zeigt, daB an diesen redundanten Orten Informationen untergebracht sein können, die für das Wesen des Erzählverlaufs von charakteristischer oder gar ästhetisch relevanter Bedeutung sind. Diesen Fall habe ich untersucht an Beispielen, wo der Raum das primäre Darstellungsobjekt ist.

Dieses Modell ist aber prinzipiell umkehrbar: Ein Autor stellt einen beliebigen Gegenstand oder Vorgang dar. Am Ende dieser Erzählphase sei dieser vollständig dargestellt. Dann ist, auch wenn der Raum mit keinem Wort erwähnt wird, eine räumliche Wirkung auf den Leser im Verlauf des Lesens festzustellen. Diesen Vorgang nenne ich Raumgenese. Da der hier entstandene Raum, gleichgülig, ob er von Zeichen unvollständig oder gar nicht vertreten wird, vollständig ist, spreche ich von einer Transposition des Nerkmals der Vollständigkeit von der zeitlichen Einheit der Erzählphase auf den Raum in Hinblick auf seine leserbezogene Wirkung.

Auf der Grundlage dieser theoretischen Ergebnisse, die den Platz des Raumbegriffes im terminologischen Apparakt der literarischen Analyse aufzeigen, wobei Raumanalyse einerseits bedeutet, daß ein dargestellter Raum befragt wird, wie er entstanden sei, andererseits aber das Erzählwerk unter dem beschränkten Blickwinkel der Raumanalyse betrachtet wird, hat der Teil der praktischen Anwendungsbeispiele Ergebnisse auf folgenden Sektoren gezeitigt: 


\section{Schichtung des Werkes}

Bei der Frage nach den relevanten Schichten des Werkes kann die Beschränkung auf den Raumaspekt als heuristisches Prinzip dienen. Hier ist von entscheidender Bedeutung, ob eine semantisch einheitliche Schicht für den Charakter des werkspezifischen Raumes verantwortlich ist, oder ob der Raum an definierbaren Stellen auftritt. Als Stellen kommen in Frage die Punkte im Handlungsablauf, an denen sich der Raum in einer besonderen Gestalt zeigt, oder die Punkte im Erzählablauf, die für die Einfügung einer Raumdarstellung geeignet sind, wobei der Raum durch seinen ungewöhnlichen Charakter oder seine hervorragenden lierkmale das Interesse auf sich zieht.

2. Die epische Integration

Unter diesem horizontalen Aspekt taucht die Frage auf, wie raumbezogene Erzählphasen in den Verlauf der gesamten Erzählung integriert sind oder wie verschiedene heterogene Erzählphasen (heterogen in bezug auf diskontinuierlichen Handlungsablauf oder thematische Uneinheitlichkeit) durch den Raum zu einer Einheit werden.

\section{3. Ästhetische Kriterien}

Unter diesem Gesichtspunkt muß entschieden werden, ob der. Raum - sein Wesen oder seine spezifisch sprachliche Gestaltung - ästhetisch relevante Kriterien trëgt oder Wirkungen ausübt, oder ob der Raum einen Beitras dazu leistet, einem ästhetisch wirksamen Objekt zum Status eines Erzählwerks zu verhelfen.

4. Spezifizierung des Erzählwerkes

Bei sprachlichen Ereignissen ist zu entscheiden, ob sie als in einem bestimmten Raum stattfindend betrachtet werden und wie sich dieses räumliche Umfeld auf die Bedeutung und den Verlauf dieses Ereignisses auswirkt, oder ob diese der Erfassung und Darstellung einer räumlichen Gegebenheit dienen. Spezifisch für das Erzählen ist die Tatsache, daß Funktionen der einen Art auch Auswirkungen auf dem anderen Sektor zeitigen.

5. Erzähltraditionen

Werden aufgrund der ausgeführten Entscheidungen kiodelle (z.B. 
Topoi, Darstellungsschemata $u_{.}$ä) freigelegt, so muß man entscheiden, ob es sich dabei um aus der Erzähltradition entnommene Modelle handelt und auf welchem Sektor dieser hodelle individuelle Ausformungen, Weiterentwicklungen und neue Ausdrucksregister zu finden sind.

Diese fünf Schritte wurden aufgrund der Erfahrungen, die bei der Analyse der vorliegenden Werke gemacht wurden, als die wirksamsten durchgeführt. Wie sie zu einem geschlossenen Untersuchungsprogranm anzuordnen sind, kann generell für das Objekt Erzählwerk nicht festgelegt werden. Die spezielle Schrittfolge muß sich nach dem jeweiligen Untersuchungsgegenstand und -ziel richten. 
Aldridge, J.W. (Hrgb.): Critiques and Essays on Modern Piction, New York 1952.

Alewyn, R.: Eine Landschaft Eichendorffs, in: Euphorion 51, 1957, 47-60.

Asher, J.A.: $\quad$ Turning Points in Kafka's Stories, in: Modern Language Review 57, 1962, 47-52.

Auerbach, E.: Mimesis, eine Geschichte der literarischen Eroberung der modernen Wirklichkeit, Bern 1946.

Bachelard, G.: Poetik des Raumes, München 1962.

Békésy, G.v.: Experiments in Hearing, übers. von E.G. Wever, New York 1960.

Bennett, E.K.: A History of the German Novel, Cambridge 1934. Berstl, H.:

Das Raumproblem in der altchristlichen Mialerei, Bonn, Leipzig 1920.

Bertalanffy, L.v.: Die Entdeckung des Raumes, in: Zeitschrift für Ästhetik 20, 1926, 307-311.

Bezzel, Ch.: Natur bei Kafka, Nürnberg 1964.

Bischof, N.: Psychophysik der Raumwahrnehmung, in: Handbuch der Psychologie I/1, 1965, 307-408.

Börsch-Supan, E.: Das Motiv des Gartenraumes in Dichtungen des 19. und frühen 20. Jahrhunderts, in: DVj 39, $1965,87-124$.

Bollnow, O.F.: Mensch und Raum, Stuttgart 1963. Bonaparte, $\mathbf{M}_{.}$: Time and the Unconscious, in: International Journal of Psycho-Analysis 21, 1940, 427-468. Bonnot, B.R.: Le roman du temps, a propos de V. Woolf et de James Joyce, in: Journal de Psycholo gie 53, 1956, 454-472.

Booth, W.C.: Distance and Point-of-view, an Essay in Classification, in: Essays in Criticism 11, 1961, 60-79.

Bourbeck, C.: Die Struktur der Zeit in heutiger Dichtung, Berlin 1956.

Brinkmann, R.: Wirklichkeit und Illusion, Tübingen 1957. Bühler, $K_{\bullet}$ : Sprachtheorie, Stuttgart ${ }^{2} 1965$. 
Buland, M.:

Burger, F.:

Cassirer, E.:

Chevalley, A.:

Claude l, P.:

The Presentation of Time in the Elizabeathan Drama, Yale University 1946.

Perspektive und Allgemeines uber Raumprobleme, in: Handbuch der Kunstwissenschaften 5 , 1913, 103-120.

Mythischer, ästhetischer und theoretischer Raum, in: Beilageheft zur Zeitschrift für Asthetik 25, 1931, 21-36.

Temps, Histoire, Roman, in: Revue de litterature comparee, Band 2, 1928, 205-241. Art poltique, connalssance du temps, Paris 1913.

Conrad-Martius, H.: Die Zeit, München 1954.

: Der Raum, München 1958.

Cowen, R.C.:

Die Dildhaftigkeit in der Lyrik Gottfried Kellers, Diss. Göttingen 1961.

Cruikshank, J.: Gide's Treatment of Time in "La Symphonie Pastorale", in: Essays in Criticism 7, 1957, $134-143$.

Daiches, D.: Dorsch, F.: Dunne, J.W.:

The Novel and the Modern World, Chicago 1960. Psychologisches Wörterbuch, Bern 1963. Buiten de grenzen van den tijd, 's-Gravenhage 1935.

Entretiens sur les notions de genèse et de structure, sous la direction de M. de Gandillac, L. Goldmann, J. Piaget, Paris 1965.

Fellmann, F.: Style formulaire und epische Zeit im Rolandslied, in: Germanisch-romanische Monatsschriften 43, 1962, 337-360.

Frank, J.: Spatial Form in the Modern Novel, in: Critiques and Essays, ed. W. Alridge, New York 1952. Fränkel, H.: Die Zeitauffassung in der archaischen griechischen Iiteratur, in: Zeitschrift für Asthetik 25, 1931, 97-117, auch in: Wege und Formen frühGriechischen Denkens, München 1955, 1-22.

Fraisse, P.: Zeitwahrnehmung und Zeitschätzung, in: Handbuch der Psychologie I/1, 1965, 656-692. 
Friedmann, N.: Point of View in Fiction, in: PMLA 70, 1955, $1160-1184$.

Frey, D.:

Gotik und Renaissance als Grundlagen der modernen Weltanschauung, Augsburg 1929.

Gerz, A.: Rolle und Funktion der epischen Vorausdeutung im mhd. Epos, Berlin 1930.

Gestalthaftes Sehen, Ergebnisse und Aufgaben der Morphologie, ed. F. Weinhand, Darmstadt ${ }^{2} 1967$.

Glassner, R.: Studien zur Geschichte des französischen Zeitbegriffs, München 1936.

Goldmann, L.: Problèmes d'une sociologie du roman, in: Cahiers internationaux de sociologie 32, 1962, 61-72.

Gombrich, E.H.: Art and Illusion, New York 1960.

Gougenheim, G.: La notion de fréquence, in: La classe de Francais, Junt 1954, 325 ff.

Griesebach, C.: Zeitbegriff und Zeitgestaltung in den Romanen Chretiens de Troyes und Hartmanns von Aue, Diss. Freiburg 1956.

Hager, $W_{\bullet}:$

tber Raumbilduns in der Architektur und in den darstellenden Künsten, in: Studium Generale 10, 1957, 630-645.

Hahn, I.:

Raum und Landschaft in Gottfrieds "Tristan", München 1963.

Hähner, R.:

Raum und Zeit im altfranzösischen Rolandslied, Diss. Freiburg 1951.

Heger, K.:

Die Bezeichnung temporal-deiktischer Begriffskategorien im französischen und spanischen Konjugationssystem, Tübingen 1963.

Hellwig, B.: Raum und Zeit im Homerischen Epos, Hildesheim 1964.

Herzog, 0.:

Zeit und Raum, das Absolute in der Kunst und Natur, Berlin 1930.

Hess, G.: Die Landschaft in Baudelaires "Fleurs du Lal", Heidelberg 1953.

Holl6s, I.:

ther die Phasen des Selbstbewußtseinsaktes, in: Zeitschrift für ärztliche Psychoanalyse 5, 1919. 93-101. 
Höllerer, W.:

Humphrey, R.:

Ivins, $W_{\bullet}:$

$-\quad:$
Jammer, $M_{\bullet}:$
Jantzen, $H_{\bullet}:$

Jaus , R . :

Jensen, H.:

Johnson, E.E.:

Kamphausen, A.:

Kayser, W.:

Kerkhoff, E.:

Kijm, J.M.:

Killy, W.:

Klages, I.:

Klein, J.:

Kleiner, J.:
Gottfried Keller: "Die Zeit geht nicht", in: Die deutsche Iyrik, hrgb. V. B.V.Wiese, Düsseldorf 1957, 2, 201-217.

Die Bedeutung des Augenblicks im modernen Romananfang, in: Romananfänge, Iiterarisches Colloquium, Berlin 1965, 344-377. Stream of Consciousness in the Modern Novel, Berkeley und Los Angeles 1962.

Art and Geometry, Cambridge Massachusetts, 1946.

Prints and Visual Communication, London 1953. Das Problem des Raumes, Darmstadt 1960. Uber den kunstgeschichtlichen Raumbegriff, Sitzungsbericht der Bayr. Ak. d. Wiss., phil.hist. Abt. 5, 1938, Neudruck Darmstadt 1962. Zeit und Erinnerung in Marcel Prousts "A la recherche du temps perdu", Heidelberg 1955. Der sprachliche Ausdruck für Zeitauffassungen, in: Archiv für die gesamte Psychologie 101, 1938, 289-336.

Tense Significance as the Time of Action, Language Diss. publ. by the Iing. Soc. of America Nr. 21, 1936.

Der Raum in der deutschen Kunst, Neumünster 1949.

Das sprachliche Kunstwerk, Bern 51959. Ausdrucksmöglichkeiten neuhochdeutschen Prosastils, Diss. Groningen, Amsterdam 1949. I'expérience du vide, in: Situation, UtrechtAntwerpen 1954, 150-171. Wirklichkeit und Kunstcharakter, München 1963. Die Sprache als Quell der Seelenkunde, Stuttgart 51959.

Das Raumerlebnis in der Lyrik Eichendorffs, in: Zeitschrift für Asthetik 29, 1935, 52-61. The Role of Time in Iiterary Genres, in: Zagadnienia rodzaj6w literackich, 2, 5-12. 
Klopfer, P.:

Knauer, K.:

Kobel, E.:
Die beiden Grundlagen des Raumschaffens, in: 1 Zeitschrift für Ästhetik 20, 1926, 311-317. Die Analyse von Feinstrukturen im sprachlichen Zeitkunstwerk, in: Mathematik und Dichtung, hrgb. v. H. Kreuzer und R. Gunzenhäuser, München ${ }^{2} 1967$.

Untersuchungen zum gelebten Raum in der mhd. Dichtung, Zürich 1950.

Krzyzanowski, J.: Henryk Sienkiewicz, in: Obraz literatury polskiej 4, Warszawa 1966, 201-240.

Kułczycka-Saloni, J.: Bolesław Prus, in: Obraz literatury polskiej 4, Warszawa $21966,145-200$.

Langen, A.:

Verbale Dynamik in der dichterischen Landschaftsschilderung des 18. Jahrhunderts, in: Zeitschrift für deutsche Philologie 70, 1948/ 49, 249-318.

Lämmert, E.: Bauformen des Erzählens, Stuttgart 1955. Lapicque, Ch.: Essais sur l'espace, l'art et la destinée, Paris 1958.

Lerle, E.: Das Raumverständnis im Alten Testament, Stuttgart ${ }^{2} 1955$.

Lessing, G.E.: Kritik und Dramaturgie, Stuttgart 1957.

Lewin, $\mathrm{K}_{\text {: : }}$

Feldtheorie in den Sozialwissenschaften,

Bern 1963.

Lohner, E.:

Thematik, Symbolik und Technik im Werk William Faulkners, Diss. Bonn 1951.

Lubbock, P.:

The Strategy of Point of View, in: Critiques and Essays on Modern Fiction, ed. W. Alridge, New York 1952, 9-30.

McLuhan, M.: Space, Time, and Poetry, in: Explorations 4, $1955,56-62$.

The Gutenberg Galaxy, Toronto 1962.

Lützeler, H.: Vom Sinn der Bauformen, Freiburg 1938.

Luxenburger, M.: Die Zeitgestaltung in Wolframs von Eschenbach "Parzival", Diss. Bonn 1949.

Maeterlinck, M.: La vie de l'espace, Paris 1928. Matz, W.:
Der Vorgang im Epos, Hamburg 1947. 
Mehmel, F.:

Nendilow, A.:

Merkel-Nipperday,

Metallmann, J.:

Metzger, W.:

Mey, H.:

Meyer, H.:

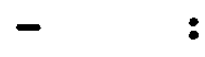

$-\quad:$

Meyerhoff, H.: Moritz, A.:

Müller, Gert:

Müller, Günther:

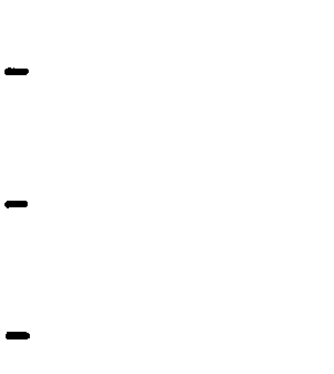

Müller, Hella:
Virgil und Appoĺonius Rhodius. Untersuchungen über die Zeitvorstellungen in der antiken epischen Erzählung, Hamburg 1940. Time and the Novel, New York 1952.

.: Gottfried Kellers "Martin Salander", Untersuchungen zur Struktur des Zeitromans, Göttingen 1959.

Problemat struktury $i$ jego dominujące stanowisko w nauce wspolczesnej, in: Kwartalnik filozoficzny 11, 1933, 332-353.

Das Raumproblem in der Psychologie, in: Studium Generale 10, 1957, 542-552.

Studien zur Anwendung des Feldbegriffs in den Sozialwissenschaften, München 1965.

Zum Problem der epischen Integration, in: Trivium 8, 1949, 299-318.

Raum und Zeit in Wilhelm Raabes Erzählkunst, in: DVj 24, 1950, 236-267, zit. nach lleuabdruc in: Zur Poetik des Romans, hrgb. v. V. Klotz, Darmstadt 1965.

Raumgestaltung und Raumsymbolik in der Erzählkunst, in: Studium Generale 10, 1957, 620-630. Time in Literature, Los Angeles 1955. Die Raumstruktur in Immermanns "Epigonen", Diss. Göttingen 1955.

Wortfeld und Sprachfeld, in: Festschrift für Ernst Otto, Berlin 1957, 155-163.

Die Bedeutung der Zeit in der Erzählkunst, Bonn 1947.

: Uber das Zeitgerüst des Erzählens, in: DVj 24: 1950, 1-31.

: Zeiterlebnis und Zeitgerüst in der Dichtung, in: Studium Generale 8, 1955, 594-601.

: Aufbauformen des Romans, Groningen-Djakarta, 1953.

Naturillusion in der Innenraumkunst des späteren 18. Jahrhunderts, Diss. Göttingen 1957. 
Müller, Karl:

Muiller, Kurt:

Ohmann, S.:

Onimus, J.:

Oppel, $\mathrm{H}_{\bullet}:$

Ott, K.A.:

Pabst, W.:

Panofsky, E.:

Parry, I.:

Pascal, R.:

Pehnt, W.:

Perpeet, J.:

Plessow, G.:

Pollmann, I.:

Poullion, J.: Poulet, G.:
Raum und Zeit in der Sprache, in: Wissenschaftliches Beiheft zur Zeitschrift des deutschen Sprachvereins 50, 69-115.

Der Aufbau figural-optischer Phänomene bei sukzessiver Reizung, Frankfurt 1963.

Theories of the Linguistic Field, in: Word 9 , 1953, 121-134.

I'Expression du temps dans le roman contemporain, in: Revue de littérature comparée, 28 , 1954, 299-317.

Die Zeitgestaltung in Hamlet, in: Jahrbuch für ̈̈sthetik 1951, 145-160.

Uber die Bedeutung des Ortes im Drama von Corneille und Racine, in: Germanisch-romanische Monatsschriften 42, 1961, 341-365.

Funktionen des Raumes in der modernen französischen Literatur, in: Universitätstage 1960, $30-46$.

Die Perspektive als "symbolische Form", in: Vorträge der Bibliothek Warburg, 4, 1924/25, 258-330.

Space and Time in Rilke's Sonnets, in: Modern Language Revue 58, 1963, 524-531.

Tense and Novel, in: liodern Language Revue 57 , 1962, 1-11.

Zeiterlebnis und Zeitdeutung in Goethes Lyrik, Tübingen 1957.

Von der Zeitlosigkeit in der Kunst, in: Jahrbuch für Ästhetik, 1951, 1-28.

Gotische Tektonik im Wortkunstwerk, liünchen 1931.

Arnot Daniel und die Entdeckung des Raumes, in: Germanisch-romanische Honatsscinriften 15, 1965, 2-14.

Temps et roman, Paris 1949.

Etudes sur le temps humain, 1-2, Plon 1950. La distance interieure, Plon 1952. 
Porzig, W.:

Ruberg, U.:
Das Wunder der Sprache, Bern 21957. Raum und Zeit im Prosa-Lancelot, München 1965.

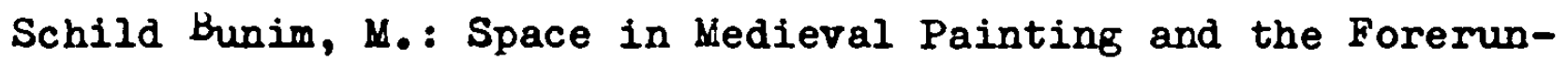
ners of Perspective, New York 1940.

Schorn, D.H.: Die Zeit in den Tristramdichtungen Eilharts und Gotfrieds. Studie zur Wirklichkeitsauffassung in mittelalterlichen Dichtungen, Diss. Köln 1952.

Schürer, 0.:

Der Bildraum in den späten Werken von Hans von Markes, in: Zeitschrift für Ästhetik 28, $1934,175-183$.

Schwerte, $\mathrm{H}_{\bullet}$ :

Simultaneität und Differenz des Wortes in Schillers "Wallenstein", in: Germanisch-romanische Monatsschriften 46, 1965, 15-25.

Seckel, D.: Hölderlins Raumgestaltung, in: Dichtung und Volkstum 39, 1938, 469-486.

Slochower, H.: Marcel Proust: Revolt against the Tyranny of Time, in: The Swannee Review 51, 1943, 370-387. Snell, B.: Arkadien, die Entdeckung einer geistigen Landschaft, in: Entdeckung des Geistes, Hamburg 21955.

Staiger, E.: Die Zeit als Einbildungskraft des Dichters, Zürich 1939.

Stanzel, F.: Die typischen Erzählsituationen, Wien-Stuttgart 1955.

Steinhoff, H.H.: Die Darstellung gleichzeitiger Geschehnisse im mittelhochdeutschen Epos, München 1964. Ströcker, E.: Die Perspektive in der bildenden Kunst, in: Jahrbuch für Ästhetik 4, 1958/59, 140-231. Stürzl, E.: Die Zeit in den Dramen J.B. Priestleys, in: Germanisch-romanische Monatsschriften 38 , 1957, 37-52.

Sviderskij, V.I.: Prostranstvo i vremja, Moskva 1958. Talon, H.A.: Time and Memory in Thakeray's "Henry Esmond", in: The Review of English Studies 13, N.S. $1962,147-156$. 
Tietgens, H.: Möglichkeit einer Zeitgestaltuntersuchung, dargestellt an Gottfried Kellers "Leute von Seldwyla", Diss. Bonn 1949.

Trier, J.: Das sprachliche Feld, in: Neue Jahrbücher für Wissenschaft und Jugendbildung 10, 1934, 428449.

Troß, E.: $\quad$ Das Raumproblem in der bildenden Kunst, München 1914.

Trzynadowski, J.: Information Theory and Literary Genres, in: Zagadnienia rodzaj6w literackich 4, ${ }^{1} 1963$, $31-45$.

Vachon, A.:

Vial, F.:

Weigand, H.:

Weimann, R.:

White, J.:

Whittacker, E.: Wölfflin, H.:

Wulff, $0 .:$

Zielinski, T.:

Zimmermann, G.:
Le temps et l'espace dans l'oeuvre de Paul Claudel, Paris 1965.

Le symbolisme Bergsonien du temps dans l'oeuvre de Proust, in: PMLA 55, 1947, 1191-1212. Die epischen Zeitverhältnisse in den Graldichtungen Chrestiens und Wolframs, in: PMLA 53, 1938, 917-950.

Erzählerstandpunkt und Point of View, Zur Geschichte und Âsthetik der Perspektive im englischen Roman, in: Zeitschrift für Anglistik und Amerikanistik 9/10, 1962, 369-416. The Birth and Rebirth of Pictorial Space, Iondon 1957.

Space and Spirit, Hindsdale (Illinois) 1948. Kunstgeschichtliche Grundbegriffe, München 1915.

Das Raumerlebnis des Naos im Spiegel der Ekphrasis, in: Byzantinische Zeitschrift 39, 1929/30, 531-539.

Die Behandlung gleichzeitiger Ereignisse im antiken Epos, in: Philologus 8, 1901, 405-449. Die Darstellung der Zeit in der mittelhochdeutschen Epik im Zeitraum von 1150-1220, Diss. Kiel 1951. 


\section{Analysierte Erzählwerke}

Andrzejewski, Jerzy: Bramy raju, Warszawa 1963.

Dąbrowska, Maria:

Noce i dnie, Warszawa 1953, 1-2.

Iwaszkiewicz, Jarosłam: Młyn nad Utratą, in: Powiadania, Warszawa 1, 1956, 261-357.

Prus, Bolesław:

Lalka, 1-2, Warszawa 1949.

Reymont, Władysław St.: Chłopi, 1-5, Warszawa 1952.

Schulz, Bruno:

Sklepy cynamonowe, Sanatorium pod klepsydrq, Kometa; Krakbw 1957.

Sienkiewicz, Henryk: Quo Vadis, Kraków 1924.

: Szkice węgłem, in: Nowele wybrane, Warszawa 1949.

Żeromski, Stefan: Utwory wbrane, 1-5, Warszawa 1961. 
28. Kunert, I.: J. U. Niemcewicz: Śpiewy historyczne, Geschichtsauffassung und -darstellung $1968,132 \mathrm{~S}$.

29. Steinke, K.: Studien aber den Verfall der bulgarischen Deklination. Das bulgarische Kasussystem zu Beginn des 13. Jahrhunderts. 1968, 7 Abb., 133 S.

30. Tschøpl, C.: Vjačeslav Ivanov. Dichtung und Dichtungstheorie. 1968, $310 \mathrm{~S}$.

31. Rehder, P.: Beitrăge zur Erforschung der serbokroatischen Prosodie. Die linguistische Struktur der Tonverlaufsminimalpaare. 1968, 247 S.

32. Kulman, D.: Das Bild des bulgarischen Mittelalters in der neubulgarischen Erzȟhiliteratur. 1968, $276 \mathrm{~S}$.

33. Burkhart, D.: Untersuchungen zur Stratigraphie und Chronologie der sudslavischen Volksepik. 1968, $549 \mathrm{~S}$.

34. Günther, H.: Das Groteske bei N. V. Gogol'. Form und Funktionen. 1968, 289 S.

35. Kazoknieks, M.: Studien zur Rezeption der Antike bei russischen Dichtern zu Beginn des XIX. Jahrhunderts. 1968, 289 S.

36. Schmidh H.: Hus und Hussitismus in der tschechischen Literatur des XIX. und XX. Jahrhunderts. 1969, $290 \mathrm{~S}$.

37. Schneider, S.: Studien zur Romantechnik Miroslav Krležas. 1969, 285 S.

38. Stephan B.: Studien zur russischen Castuška und ihrer Entwicklung. 1969, 358 S.

39. Girke, W.: Studien zur Sprache N. S. Leskovs. 1969, VIII, 220 S.

40. Mareš, F. V.: Diachronische Phonologie der Ur- und Frühslavischen. 1969, 126 S.

41. Wosien, M.-G.: The Russian Folk-Tale. Some Structural and Thematic Aspects. 1969, 237 S.

42. Schula, R. K.: The Portrayal of the German in Russian Novels. Goncarov, Turgenev, Dostoevskij, Tolstoj. 1969, VI, 215 S.

43. Baudisch, G.: Das patriarchale Dorf im Erzählwerk von Janko M. Veselinovic. 1969, 225 S.

44. Stollting, W.: Beitrage zur Geschichte des Artikels im Bulgarischen. 1970, 296 S.

45. Hucke, G.; Jurij Fedorovii Samarin. Seine geistesgeschichtliche Position und politische Bedeutung, 1970, 263 S.

46. Höcherl, A.: Zur Ubersetzungstechnik des altrussischen "Judischen Krieges" des Josephus Flavius. 1970, 183 S.

47. Sappok, Ch.: Die Bedeutung des Raumes fur die Struktur des Erzählwerks, aufgezeigt an Beispielen aus der polnischen Erzählliteratur. 1970, 154 S.

48. Guski, A.: M. Ju. Lermontovs Konzeption des literarischen Helden. 1970, 225 S. 